\title{
Flux Tube Profiles at High Temperature
}

\author{
Dissertation \\ zur Erlangung des Doktorgrades \\ an der \\ Fakultät für Physik \\ der Universität Bielefeld
}

vorgelegt von

Sodbileg Chagdaa

March 2008 


\section{Contents}

1. Introduction 2

2. Introduction to Lattice Gauge Theories 5

2.1. Discretizing Space-Time . . . . . . . . . . . . . . 5 5

2.2. The Path Integral Approach to Quantization and The Partition Function 6

2.3. Finite Temperature Lattice Gauge Theories . . . . . . . . . . . . . 8

2.3.1. Polyakov Loop . . . . . . . . . . . . . . . . . . . . . . . . . 10

2.3.2. Spontaneous Breakdown of the Center Symmetry and the Deconfinement Phase Transition . . . . . . . . . . . . . . 11

2.3.3. Determination of the Transition Temperature . . . . . . . . . . 12

3. Heavy Quark Potentials 15

3.1. Heavy Quark Potentials at Zero Temperature . . . . . . . . . . . . . 15

3.2. Heavy Quark Potentials at Finite and High Temperature . . . . . . . . . 16

4. Flux Tube Model 23

4.1. Dual Superconductor Picture of Confinement . . . . . . . . . . . . . . 24

4.2. Lattice Measures of the Flux Tube . . . . . . . . . . . . . . . . . . 25

4.3. Flux Tube at Zero Temperature . . . . . . . . . . . . . . . . . . 27

5. Measurements on the Lattice 30

5.1. Update and Details of the Simulation . . . . . . . . . . . . . . . . . 30

5.2. Error Reduction . . . . . . . . . . . . . . . . . . 32

5.2.1. The Reference Point Method . . . . . . . . . . . . . . . . 32

5.2.2. Noise Reduction by Link Integration . . . . . . . . . . . . . . . 34

5.3. From the Lattice to the Continuum . . . . . . . . . . . . . . . . . . 40

6. Analysis for Flux Tube Profiles at Finite Temperature 43

6.1. Distribution Surface . . . . . . . . . . . . . . . . 43 
6.2. Longitudinal Profiles . . . . . . . . . . . . . . . . . . . . . . . . . . . 48

6.2.1. Results on the $16 \times 8^{2} \times 4$ lattice . . . . . . . . . . . . 49

6.2.2. Results on the $24 \times 12^{2} \times 6$ lattice . . . . . . . . . . . 53

6.2.3. Finite $a$ Effects . . . . . . . . . . . . . . . . 55

6.3. Transverse Profiles . . . . . . . . . . . . . . . . 56

6.4. Energy and Action densities . . . . . . . . . . . . . . . 60

6.5. Fit to the Transverse Distribution . . . . . . . . . . . . . . . 68

6.6. Width of the Flux Tube . . . . . . . . . . . . . . . 70

6.7. Disappearance of The Flux Tube above $T_{c} \ldots \ldots \ldots$. . . . . . . 72

$\begin{array}{ll}\text { 7. Conclusions } & 78\end{array}$

$\begin{array}{ll}\text { Appendix } & \mathbf{8 0}\end{array}$

A. $S U(2) \ldots \ldots \ldots \ldots \ldots \ldots \ldots \ldots \ldots$

B. Maxwell and Proca equations . . . . . . . . . . . . . . . . . . . 82

C. Link integration for $S U(2) \ldots \ldots \ldots \ldots$. . . . . . . . . . . 84

- Bibliography $\quad 86$

- Acknowledgements $\quad 89$ 


\section{Introduction}

The standard model of particle physics is a theory that describes three of the four known fundamental interactions between the elementary particles that make up all matter. It is a quantum field theory developed between 1970 and 1973 which is consistent with both quantum mechanics and special relativity. The standard model is a grouping of two major theories quantum electroweak and quantum chromodynamics which provides an internally consistent theory describing interactions between all experimentally observed particles.

According to the Standard Model, the basic classification of fundamental particles in nature is into hadrons which experience the strong interaction, leptons which do not, and thirdly, the quanta of the interaction fields. All hadrons are bound states of particles called quarks, and leptons themselves are considered to be fundamental, so the leptons and the quarks form the basic building blocks of all matter in the universe.

In this model quarks come in six different flavours, up $(u)$, down $(d)$, strange $(s)$, charmed $(c)$, bottom $(b)$ and top $(t)$. They also have a hidden three valued degree of freedom known as colour: each quark flavour comes in three colours. The forces through which quarks interact are the strong forces of attraction or repulsion between these colour charges. The strong forces are carried by massless bosons called gluons.

The theory of quark interactions arising from the exchange of gluons is called Quantum Chromodynamics (QCD). The local gauge group is $S U(3)$ generated by three colour charges, the field quanta are eight massless spin 1 colour carrying gluons. One of the most striking physical properties of QCD is confinement of quarks in a hadron so that only colour singlets can be produced and observed. In high energy atomic collisions, we can split an atom into its constituents, atomic nucleus and electrons. In high energy nucleus-nucleus collisions, we can split a nucleus into its constituents, neutrons and protons. But in high energy hadron-hadron collisions, a hadron is not split into 
its constituents, quarks. A hadron-hadron collision results not into free quarks but into hadrons.

Quark confinement is reflected in the attractive, linearly rising potential of a static quark and an antiquark $(q \bar{q})$ pair. Since the potential linearly confines, it would take infinite energy to separate such pairs. The study of confinement has always been one of the most challenging topics in particle physics and appears to play a role solely for the unbroken non-Abelian gauge theory of strong interactions.

Such a linear rise with slope $\sigma$ can be explained by the formation of a flux tube between the $q \bar{q}$ pair with constant energy per length $\sigma$. In the flux tube picture, the chromoelectric field originating from a quark does not spread uniformly in space, but is squeezed into tubes of constant cross section. Since confinement is related to flux tube formation, to understand confinement essentially means to understand the properties of the flux tube.

At low temperature and density, quarks and gluons appear only as constituents of hadrons because of confinement. However, QCD lattice simulations predict that above a certain temperature or density, nuclear matter undergoes a phase transition after which quarks and gluons are deconfined from hadrons and form a new state of matter called quark-gluon plasma (QGP). Investigating the behaviour of matter in the vicinity of the critical temperature of the deconfinement transition and at high temperature is a major goal of current and planned heavy ion collision experiments.

Motivated by the successful flux tube analyses at zero temperature $[1,2,3,4]$, interesting questions arise at finite or high temperature, concerning the behaviour of the flux tube when QCD undergoes a phase transition. What steps will the flux tube pass through and how does it behave to go to the quark-gluon plasma phase? To have answers to these questions we need to do the finite or high temperature study. To see how the flux tube melts into the quark-gluon plasma phase one has to look into the details of the interaction between quark pairs studying the distribution of the chromoelectric and chromomagnetic components of gluon fields which compose energy and action density of the flux tube. This investigation may also shed more light to our current understanding about the confinement problem. 
The main goal of this work is a more detailed quantitative analysis of the flux tube picture at high temperature. In particular we shall see how the shape and size of the flux tube change when QCD undergoes a phase transition from the hadronic phase to a QGP.

The organization of this work is as follows.

Chapter 2 will be devoted to a brief introduction to Lattice Gauge Theory. It also contains the lattice formulation of finite temperature field theory. Chapter 3, which is about the heavy quark potential, describes the main motivation of doing our finite temperature investigation of flux tube profiles. In Chapter 4 we present the flux tube model and its lattice formulation, which is our object to be investigated and collect its zero temperature results. Chapter 5 presents the details of the simulation, error reductional techniques and scaling relation. In Chapter 6 we present and discuss our high temperature results on various profiles of the flux tube. Finally Chapter 7 will give some concluding remarks. 


\section{Introduction to Lattice Gauge Theories}

\subsection{Discretizing Space-Time}

Quantum Chromodynamics, which is believed to be the fundamental theory of strong interactions, is a field theory of basic quark and gluon constituents. At short distances QCD is weakly coupled and can be studied analytically using perturbation theory, while at large distances it is strongly coupled and in most cases it can not be studied analytically. In order to study physical processes that are controlled by large-distance, nonperturbative effects of QCD, in 1974 Kenneth Wilson introduced Lattice Gauge Theory in which the space-time continuum is discretized on a lattice with lattice points or sites $x$. In this Euclidean lattice formulation [5], after a Wick rotation (to Euclidean space), the quantization is performed via the path integral formalism. We will follow the Euclidean formulation with a space-time lattice spacing a.

In a continuum notation the QCD Lagrangian is given as

$$
\mathcal{L}=\mathcal{L}_{G}+\mathcal{L}_{F}
$$

where

$$
\begin{gathered}
\mathcal{L}_{G}=-\frac{1}{4} F_{\mu \nu}^{a}(x) F^{a \mu \nu}(x), \\
\mathcal{L}_{F}=\bar{\psi}(x)\left(i \gamma^{\mu} D_{\mu}-M_{0}\right) \psi(x) .
\end{gathered}
$$

$\mathcal{L}_{G}$ and $\mathcal{L}_{F}$ are the Lagrangian densities of the $S U(N)$ pure gauge theories and matter fields of mass $M_{0}$, respectively and

$$
F_{\mu \nu}^{a}(x)=\partial_{\mu} A_{\nu}^{a}(x)-\partial_{\nu} A_{\mu}^{a}(x)+g f^{a b c} A_{\mu}^{b}(x) A_{\nu}^{c}(x),
$$




$$
D_{\mu}=\partial_{\mu}-i g \frac{\lambda_{a}}{2} A_{\mu}^{a}(x)
$$

Here $A_{\mu}^{a}$ are the gauge fields $\left(a=1,2, \ldots, N^{2}-1\right), g$ is the gauge coupling constant and $f^{a b c}$ are the structure constants of the $S U(N)$ group and $\gamma^{\mu}$ are $4 \times 4$ Dirac matrices satisfying the following anticommutation relations

$$
\left\{\gamma^{\mu}, \gamma^{\nu}\right\}=2 g^{\mu \nu}
$$

In the lattice formulation the quark fields are only defined at the sites of the lattice. Two adjacent sites are connected by an oriented link $n, \mu$. Instead of a vector potential as in the continuum case, the gauge field variables are defined on the links of the lattice and correspond to the parallel transport along the edge which takes on values in the Lie group. Hence to simulate QCD, for which the Lie group is $S U(3)$, there is a $3 \times 3$ special unitary matrix defined on each link. The gauge connection $U_{\mu}(n)$, the so-called link variable leaving site $n$ in direction $\mu$, replaces the continuum gauge field $A_{\mu}(x)$ in the way

$$
U_{\mu}(n)=\exp \left(i g a \sum_{a=1}^{N^{2}-1} \lambda^{a} A_{\mu}^{a}(n)\right) .
$$

The product of four links, enclosing an elementary square, is a plaquette. For simplicity, we adopt an isotropic lattice with equal lattice spacing $a$ in all directions.

\subsection{The Path Integral Approach to Quantization and The Partition Function}

A convenient way to write down the lattice theory is in the Euclidean path integral framework. Since a detailed introduction on the path integral formulation of quantum field theory is in many books about lattice gauge theories, e.g. in [6], we will not discuss it in such detail.

The expectation values of observables in QCD can be expressed by the path integral over the link variable, $U$, and the quark fields, $\psi$, as

$$
\langle\mathcal{O}\rangle=\frac{1}{Z} \int[d U][d \bar{\psi} d \psi] \mathcal{O} \quad \exp \left[-S_{G}-S_{F}\right]
$$


Here

$$
Z=\int[d U][d \bar{\psi} d \psi] \exp \left[-S_{G}-S_{F}\right]
$$

is the partition function of an $S U(N)$ gauge theory interacting with matter fields written in the Euclidean path-integral formulation and $S_{G}$ and $S_{F}$ respectively are the discretised gauge and fermion actions which are

$$
\begin{array}{r}
S_{G}=\frac{2 N}{g^{2}} \sum_{P}\left[1-\frac{T r}{2 N}\left(U_{P}+U_{P}^{+}\right)\right], \\
S_{F}=\left(M_{0}+4 r\right) \sum_{n} \sum_{a=1}^{N} \bar{\psi}^{a}(n) \psi^{a}(n) \\
-\frac{1}{2} \sum_{n, \mu} \sum_{a=1}^{N}\left[\bar{\psi}^{a}(n)\left(r-\gamma_{\mu}\right) \psi^{a}(n+\hat{\mu})\right. \\
\left.+\bar{\psi}^{a}(n+\hat{\mu})\left(r+\gamma_{\mu}\right) \psi^{a}(n)\right] .
\end{array}
$$

where $U_{P}$ stands for the product of link variables around the boundary of a plaquette $P$ taken in the counterclockwise direction.

In continuum QCD

$$
S_{G}^{(c o n t)}=\frac{1}{4} \int d^{4} x F_{\mu \nu}^{a} F_{\mu \nu}^{a}
$$

where $F_{\mu \nu}^{a}$ is related to the colored gauge potentials by Eq. 2.3. Hence, in contrast to the Abelian case, the pure gauge sector of QCD describes a highly non-trivial interacting theory, which involves tripel and quartic interactions of $A_{\mu}^{a}$. This is the reason why a study of the pure gauge sector of QCD is of great interest. In fact, the self-couplings of the gauge potentials are believed to be responsible for quark confinement. The first non-abelian gauge theory was proposed by Yang and Mills (1954) and was based on $S U(2)$. For this reason one usually refers to Eq. 2.3 or 2.5 as the Yang-Mills action [6].

Fermionic and gluonic actions on the lattice are invariant under local gauge transformations $G(n)$

$$
\begin{gathered}
\psi(n) \rightarrow G(n) \psi(n) \\
\bar{\psi}(n) \rightarrow \bar{\psi}(n) G^{-1}(n)
\end{gathered}
$$




$$
\begin{gathered}
U_{n, n+\hat{\mu}} \rightarrow G(n) U_{n, n+\hat{\mu}} G^{-1}(n+\hat{\mu}) \\
U_{n+\hat{\mu}, n} \rightarrow G(n+\hat{\mu}) U_{n+\hat{\mu}, n} G^{-1}(n) .
\end{gathered}
$$

The lattice thus provides a gauge invariant regularization scheme. In the theory, unlike in the real world, we can vary the quark masses, the number of colours, the number of flavours, the temperature, the volume, the space time dimension of our lattice-universe. In order words, lattice gauge theory offers the laboratory to test non-perturbative phenomena arising, for instance, in the high temperature regime.

Lattice formulation of the field theory is equivalent to a statistical mechanics system and can be studied using Monte Carlo numerical simulations. So we can call upon all our experience and intuition of statistical mechanics to solve problems in quantum field theory.

With a finite lattice there are a finite number of variables. By changing $\beta=2 \mathrm{~N} / \mathrm{g}^{2}$ one can control the lattice spacing $a$ throughout the simulations (see Section. 5.3). Since nonabelian gauge theories are asymptotically free, the continuum limit corresponds to infinite $\beta$. After the simulations are performed for a set of values of the lattice spacing $a$ and systems of several sizes $V$, continuum quantities can be recovered by taking the limits $V \rightarrow \infty, a \rightarrow 0$.

\subsection{Finite Temperature Lattice Gauge Theories}

In this section we will give some formula that describe the behaviour of hadronic matter at finite temperature as predicted by QCD. As the temperature enters the theory we have to define expectation values of the thermodynamical observables from the partition function

$$
Z_{Q C D}=\int[d U][d \bar{\psi} d \psi] e^{-S_{Q C D}^{(\beta)}[U, \psi, \bar{\psi}]}
$$

$S_{Q C D}^{(\beta)}$ is the finite-temperature action

$$
S_{Q C D}^{(\beta)}=\int_{0}^{\beta} d \tau \int d^{3} x \mathcal{L}
$$


where $\beta=1 / T$ is the inverse temperature here. Correspondingly, if $N_{\tau}$ is the number of lattice sites along the temporal direction, the temperature is

$$
T=1 / N_{\tau} a(\beta)
$$

A finite temperature then implies that $N_{\tau}$ of the $N_{\sigma}^{3} \times N_{\tau}$ lattice has to be shorter than $N_{\sigma}$. Also we have to impose periodic boundary conditions in the temporal direction, while the boundary conditions in the other direction can be chosen freely. Dirac fields are subjected to antiperiodic boundary condition. Thus at finite temperature the lattice is compactified in the euclidean time direction, with the link variables satisfying the boundary condition

$$
U_{\mu}(\vec{n}, \beta)=U_{\mu}(\vec{n}, 0)
$$

where $\beta$ is the inverse temperature measured in lattice units.

Given the partition function one can proceed to study the behaviour of thermodynamical observables as a function of temperature and to determine the critical properties of the theory. For instance, the energy density and pressure are defined by the partition function in the usual way

$$
\begin{gathered}
\epsilon=-\frac{1}{V} \frac{\partial}{\partial \beta}(\ln Z)_{V} \\
p=\frac{1}{\beta} \frac{\partial}{\partial V}(\ln Z)_{\beta} .
\end{gathered}
$$

These continuum expressions must be translated on the lattice into expectation values of gauge invariant expressions constructed from the link variables. According to Eq. 2.5 , to keep the physical volume $V$ or temperature $T$ fixed while varying, respectively, the temperature or volume, we must be able to vary independently the extension of the lattice in the time and space directions. For a given lattice this can be done by choosing different lattice spacings $a$ and $a_{\tau}$ along the space and time directions.

In the following we will restrict ourselves to $S U(N)$ pure gauge theory. Then the partition function has the form

$$
Z=\int_{p e r} D U e^{-S_{G}[U]}
$$




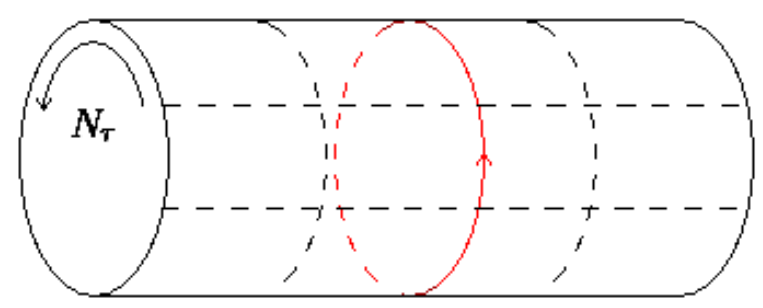

Figure 2.1.: Two dimensional picture of a loop winding around the time direction.

As we have stated in the introductory section the pure $S U(N)$ Yang Mills theory confines a static quark and an antiquark pair at low temperatures and the question is whether confinement persists as the temperature is raised, or whether there exists a critical temperature where deconfinement takes place. To have an answer to this question one needs non-perturbative observables.

\subsubsection{Polyakov Loop}

Due to the finite extension in the time direction the Wilson loop no longer plays the role to determine the potential of a static $q \bar{q}$ pair. It will be replaced by Polyakov loops which are the relevant variables in the physics of deconfinement for pure gauge theories. The Polyakov loop is a gauge-invariant quantity, which is the trace of the product of link variables along topologically non-trivial loops winding around the time direction. In Fig. 2.1 we show a two-dimensional picture of the simplest loop we can construct, located at some spatial lattice site $\vec{n}$.

Its mathematical expression is

$$
L(\vec{n}) \equiv \frac{1}{N_{c}} \operatorname{Tr} \prod_{\tau=1}^{N_{\tau}} U_{4}(\vec{n}, \tau)
$$

The Polyakov loop is invariant under gauge transformations:

$$
\begin{gathered}
U_{4}(\vec{n}, \tau) \rightarrow G(\vec{n}, \tau) U_{4}(\vec{n}, \tau) G^{-1}(\vec{n}, \tau+1) \\
G(\vec{n}, 1)=G\left(\vec{n}, N_{\tau}+1\right) .
\end{gathered}
$$

As we will see in the following section the expectation value of the Polyakov loops has a simple physical interpretation. 


\subsubsection{Spontaneous Breakdown of the Center Symmetry and the Deconfinement Phase Transition}

As a result of the periodic structure of the lattice, pure $S U(N)$ gauge action has not only local gauge invariance, but also global $Z(N)$ symmetry. Gauge transformations related to this symmetry can be expressed as

$$
U_{4}(\vec{n}, \tau) \rightarrow z U_{4}(\vec{n}, \tau), \quad \forall \vec{n}, \quad \tau \quad \text { fixed }
$$

where $z \in \mathcal{C}$. The elements belonging to the center $\mathcal{C}$ of the $S U(N)$ group are given as $\exp (2 \pi i n / N), n=0, \ldots, N-1$. While the lattice action of the pure $S U(N)$ gauge theory is invariant under above transformation, the Polyakov loop is not invariant and transforms non-trivially:

$$
L(\vec{n}) \rightarrow z L(\vec{n})
$$

Because the Polyakov loop has a special feature associated to the spontaneous breaking of the center symmetry this quantity can be used as an order parameter to describe the high temperature regime, which is characterized by the breaking of the global symmetry with respect to the center of the group.

The free energy, $F_{q}$, of an isolated static quark is given by the average of the Polyakov loop over the lattice by the following way

$$
e^{-F_{q} / T}=\langle L(\vec{n})\rangle=\langle L\rangle .
$$

In the confined $Z(N)$ symmetric phase

$$
\langle L\rangle=0
$$

while

$$
\langle L\rangle \neq 0
$$

in the deconfined phase. Thus the expectation value of the Polyakov loop evaluated in a pure gluonic medium becomes a signal for distinguishing the confined phase from the deconfined, center symmetry broken phase. 
The free energy of a quark and antiquark located at different sites of the lattice can be obtained from the correlation function of two opposite oriented Polyakov loops,

$$
\Gamma(\vec{n}, \vec{m})=\left\langle L(\vec{n}) L^{+}(\vec{m})\right\rangle
$$

where $\vec{n}$ and $\vec{m}$ are the site positions. Thus it is easy to show that the free energy $F_{q \bar{q}}(\vec{n}, \vec{m})$ of a static $q \bar{q}$ pair is given by

$$
\Gamma(\vec{n}, \vec{m})=e^{-\beta F_{q \bar{q}}(\vec{n}, \vec{m})} .
$$

Now it is well known that $S U(3)$ pure gauge theory undergoes a first order phase transition, while $S U(2)$ gauge theory undergoes a second order continuous phase transition. Continuous phase transitions are easier to study than first order transitions due to the absence of latent heat, and they have been discovered to have many interesting properties.

\subsubsection{Determination of the Transition Temperature}

As will be worked out in more detail in Section 5.3 of Chapter 5 the lattice spacing is a function of the coupling constant $\beta=2 \mathrm{~N} / \mathrm{g}^{2}$. Moreover, the temperature $T$ of a lattice with temporal extent $N_{\tau}$ is given by

$$
T=\frac{1}{N_{\tau} a(\beta)}
$$

Thus, at fixed $N_{\tau}$ one can vary the temperature by changing the coupling constant.

Correspondingly, the critical temperature $T_{c}$ of a phase transition is determined from a critical coupling $\beta_{c}$

$$
T_{c}=\frac{1}{N_{\tau} a\left(\beta_{c}\right)}
$$

One has to determine the critical couplings, where transitions occur, on a lattice with temporal extent $N_{\tau}$. The deconfining transition point in the coupling constant values for two values of $N_{\tau}$ has been identified through the location of the peak in the susceptibility, $\chi_{L}$, of the Polyakov loop using Ferrenberg-Swendsen reweighting technique [7].

The Ferrenberg-Swendsen reweighting technique [7] we used to find the critical $\beta$ value is especially important when the behavior of the system displays sharp peaks, such as 


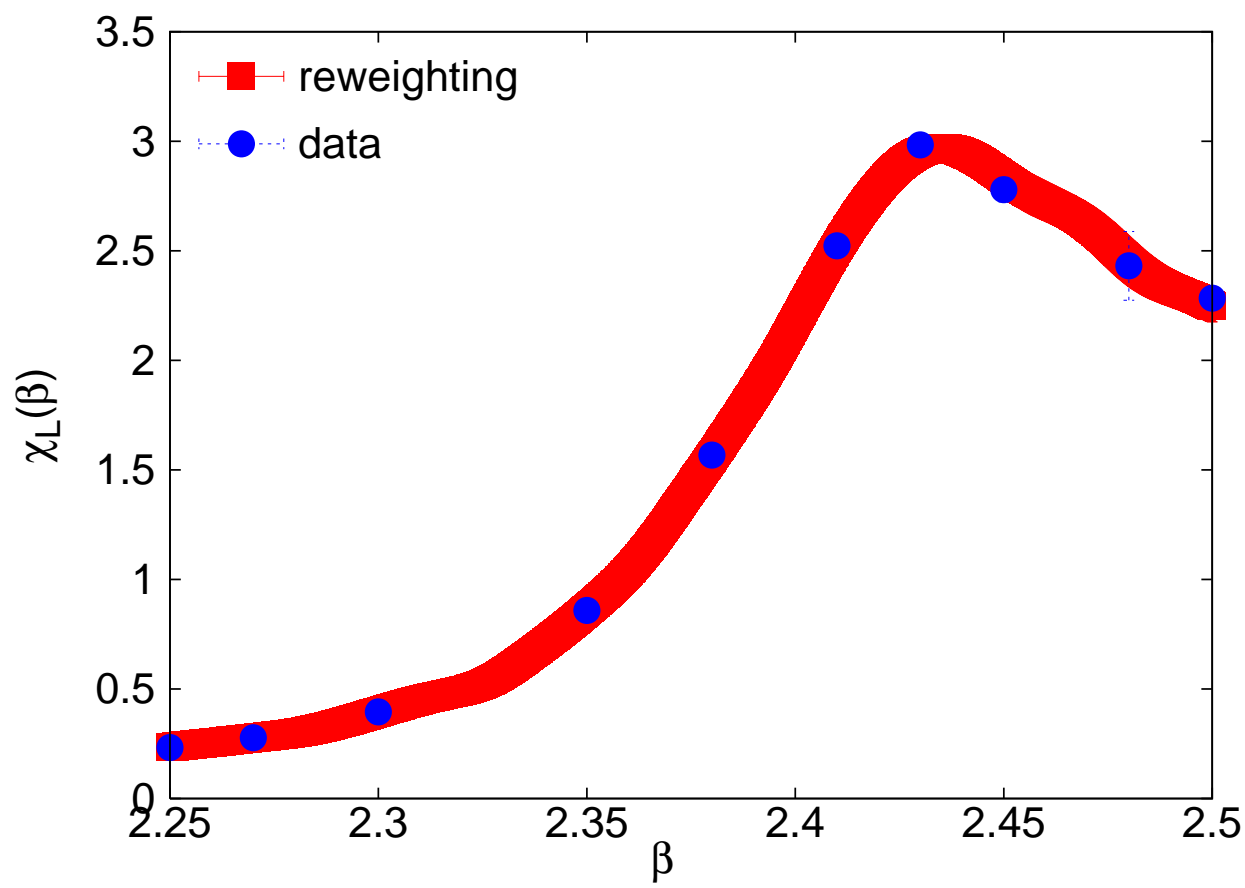

Figure 2.2.: Polyakov loop susceptibility, $\chi_{L}$, against coupling constant $\beta$ for a $12^{3} \times 6$ lattice.

near those first and second order phase transitions.

If we calculate the Polyakov loop susceptibility

$$
\chi_{L}=N_{\sigma}^{3}\left(\left\langle L^{2}\right\rangle-\langle L\rangle^{2}\right)
$$

where $N_{\sigma}^{3}$ is a factor of lattice volume, for every $\beta$ to look for the critical $\beta$, the result is a set of discrete points, none of which is exactly at the peak. With the algorithm one can accurately determine the peak position where a transition occurs. In other words, the algorithm does a weighted interpolation between those discrete points that are plotted from data. In Fig. 2.2 the Polyakov loop susceptibility, $\chi_{L}$, is plotted for several values of $\beta$, which are near the transition for $N_{\tau}=6$.

In Fig. 2.2 the blue discrete points are $\chi_{L}$ obtained from our data. As we see from these values it seems that the critical $\beta$ should be somewhere between $2.40-2.45$, but it is difficult to locate an exact position of the peak where the transition occurs, if there was no interpolating curve drawn with the red square points. The position of the peak 
has been determined with high accuracy using the reweighting method to be

$$
\begin{array}{cc}
\beta_{c}=2.2985 \pm 0.0006, & N_{\tau}=4 \\
\beta_{c}=2.434 \pm 0.002, & N_{\tau}=6 .
\end{array}
$$

Once we know the critical couplings on our two lattices with temporal extents $N_{\tau}=4$ and 6, using Eq. 2.17 we can determine the corresponding critical temperatures. 


\section{Heavy Quark Potentials}

\subsection{Heavy Quark Potentials at Zero Temperature}

As we have already mentioned the confining property of QCD manifests itself in the long range behaviour of the heavy quark potential.

In non-abelian gauge field theories, the coupling constant decreases at short distances. In the familiar Abelian theory of QED, one has an intuitive understanding of the decrease of the effective coupling constant at long distance as being due to the dielectric screening by the cloud of virtual electron-positron pairs. For non-Abelian gauge theories, we have to understand an anti-screening effect. The anti-screening effect is due to the self-couplings of the gauge potentials. Thus the exploring of the heavy quark potential in detail is important for the understanding about confinement and deconfinement. At zero temperature the potential of a system that contains two heavy quarks rises linearly at large distances,

$$
V_{q \bar{q}}(R)=V_{0}-\frac{\pi}{12} \frac{1}{R}+\sigma R
$$

where $V_{0}$ denotes the self-energy of the quark lines, $\sigma$ is the string tension which forces the quarks and gluons to be confined to a hadronic bag and the Coulomb-like term $1 / R$ stems from fluctuations of the string [8]. The static $q \bar{q}$ potential in lattice units, defined as the ground state energy of the system, can simply be expressed as a function of the Wilson loop

$$
V_{q \bar{q}}(R)=-\lim _{\hat{T} \rightarrow \infty} \frac{1}{\hat{T}} \ln W(R, \hat{T})
$$

where $W(R, \hat{T})$ is the expectation value of the Wilson loop with spatial and temporal extension $R$ and $\hat{T}$, respectively. The confining potential is spin and flavour independent. 
Since the Wilson loops can be calculated accurately, the analysis of the heavy quark potential and the string tension is useful to check scaling and thereby determine the lattice spacing $a(\beta)$. The string tension extracted from the long distance part of the heavy quark potential can be used to test the scaling of the dimensionless ratios of physical quantities [10].

\subsection{Heavy Quark Potentials at Finite and High Temperature}

It is expected that fundamental forces between quarks, which are mediated by gluons, change because of the additional interaction of gluons with the constituents of the thermal bath. The modifications of these forces due to the temperature change can be investigated from the analysis of the temperature dependence of a heavy quark potential [11].

For nonvanishing temperatures below the critical temperature $T_{c}$, a temperature-dependent potential has been computed [12], which can be expressed as

$$
\begin{array}{r}
V(R, T)=V_{0}-\left[\frac{\pi}{12}-\frac{1}{6} \arctan (2 R T)\right] \frac{1}{R}+ \\
+\left[\sigma-\frac{\pi}{3} T^{2}+\frac{2}{3} T^{2} \arctan \left(\frac{1}{2 R T}\right)\right] R+\frac{T}{2} \ln \left[1+(2 R T)^{2}\right] .
\end{array}
$$

In the limit $R \gg 1 / T$, this goes over into

$$
V(R, T)=V_{0}+\left[\sigma-\frac{\pi}{3} T^{2}\right] R+T \ln (2 R T) .
$$

In Eqs. 3.1 and $3.2 \sigma$ is the zero temperature string tension. However, one may also allow for a temperature dependent string tension $\sigma(T)$. Such a string tension was computed by means of a $1 / D$ expansion $[13]$

$$
\frac{\sigma(T)}{\sigma(0)}=\left(1-\frac{T^{2}}{T_{c}^{2}}\right)^{1 / 2}
$$

where $T_{c}$ was obtained as

$$
T_{c}^{2}=\frac{3}{\pi(D-2)} \sigma(0)
$$

In the case of $S U(3)$ [14] it is found that the string tension decreases with rising temperature but retains a finite value at the deconfinement transition, while in colour $S U(2)$ 
[15], which exhibits a second order transition, the temperature dependent string tension $\sigma(T)$ vanishes $\sim\left(\beta_{c}-\beta\right)^{\nu}$, with a critical exponent $\nu$ taking its three-dimensional Ising value of 0.63 .

There are several $q \bar{q}$ potentials that can be studied, since the $q \bar{q}$ pair can be in a singlet or an octet state at high temperature. We denote these potentials by $V_{1}(\vec{R}, T)$ and $V_{8}(\vec{R}, T)$, and identify them with the free energy of the system [6]

$$
\left(\operatorname{Tr} e^{-H / T}\right)_{(l)}=e^{-V_{l}(\vec{R}, T) / T} .
$$

Here the trace is taken over all states of the system with a heavy $q \bar{q}$ pair in the singlet $(l=1)$ or octet $(l=8)$ state, separated by a distance $\vec{R}$. This expression is degenerate in the colour magnetic quantum numbers of the $q \bar{q}$ system. We now define the so called colour averaged potential by taking the average of the above expression over the two possible states weighted with their degeneracy

$$
e^{-V(\vec{R}, T) / T}=\frac{1}{9}\left(e^{-V_{1}(\vec{R}, T) / T}+8 e^{-V_{8}(\vec{R}, T) / T}\right) .
$$

The colour averaged potential between a heavy quark and an anti-quark pair at finite temperature is computed from two Polyakov loop correlations

$$
\left\langle L(\overrightarrow{0}) L(\vec{R})^{\dagger}\right\rangle=e^{-V(|\vec{R}|, T) / T}
$$

where $L(\vec{x})$ denotes the Polyakov loop at spatial coordinates $\vec{x}$.

As a by product we have computed the potential at various temperatures. The results are in Fig. 3.1.

As temperature increases the potential decreases due to the temperature dependent string tension but keeps rising as the $q \bar{q}$ separation increases. The slope of the potential decreases as temperature increases. This means that the string tension is no longer constant but is $T$-dependent and becomes smaller close to $T_{c}$.

The potentials have first been fitted to fit ansatz Eq. 3.1 allowing for a temperature dependent string tension $\sigma(T)$. The fit results are displayed in the Table. 3.1.

One can compare the resulting string tension values with zero temperature string tension values, obtained from renormalization group inspired interpolation ansatz to data from 


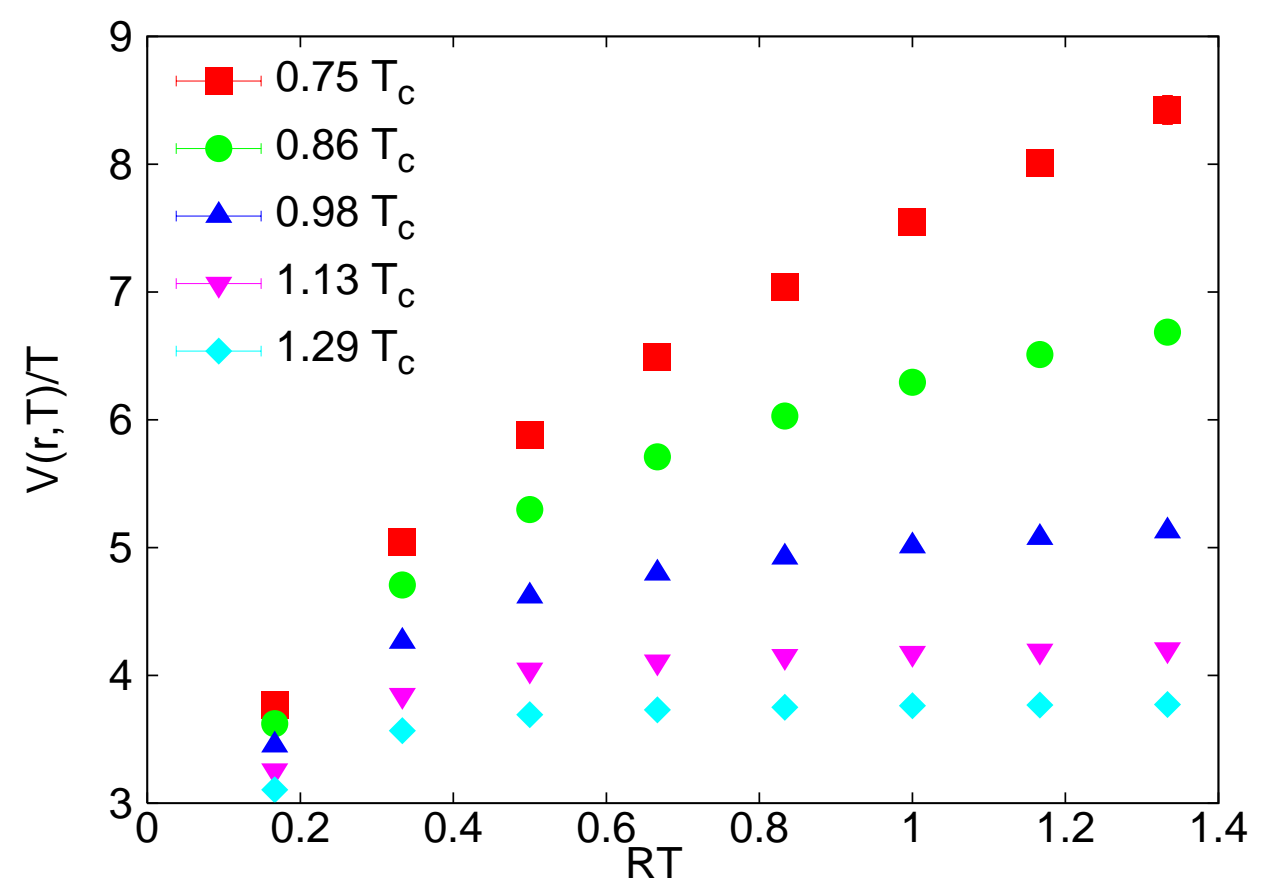

Figure 3.1.: The heavy quark potentials as a function of $q \bar{q}$ separation at various temperatures. The potentials and $q \bar{q}$ separations are in units of the temperature. The lattice size was $24 \times 12^{2} \times 6$.

\begin{tabular}{|c|c|c|c|c|c|c|}
\hline$N_{\tau}$ & $\beta$ & $T / T_{c}$ & $a V_{0}$ & $a^{2} \sigma$ & $\chi^{2}$ & $<\chi^{2}>$ \\
\hline \hline \multirow{6}{*}{6} & 2.31 & 0.65 & $0.74(2)$ & $0.120(4)$ & 0.1 & 0.04 \\
& 2.35 & 0.75 & $0.7479(6)$ & $0.0846(5)$ & 37 & 6.2 \\
& 2.39 & 0.86 & $0.7601(6)$ & $0.0491(5)$ & 20 & 3.3 \\
& 2.43 & 0.98 & $0.7688(7)$ & $0.0108(4)$ & 32 & 5.3 \\
\hline \hline
\end{tabular}

Table 3.1.: Results for the string tension from fits with Eq. 3.1.

\begin{tabular}{|c|c|c|c|}
\hline$N_{\tau}$ & $\beta$ & $T / T_{c}$ & $a^{2} \sigma$ \\
\hline \hline \multirow{6}{*}{6} & 2.31 & 0.65 & 0.128 \\
& 2.35 & 0.75 & 0.099 \\
& 2.39 & 0.86 & 0.076 \\
& 2.43 & 0.98 & 0.057 \\
\hline \hline
\end{tabular}

Table 3.2.: Results in Fig. 5.6 in the form of table. 


\begin{tabular}{|c|c|c|c|c|c|c|c|}
\hline$N_{\tau}$ & $\beta$ & $T / T_{c}$ & $a V_{0}$ & $a^{2} \sigma(T)$ & $\gamma$ & $\chi^{2}$ & $\left\langle\chi^{2}\right\rangle$ \\
\hline \hline \multirow{6}{*}{6} & 2.31 & 0.65 & $0.755(125)$ & $0.106(48)$ & $0.55(1.42)$ & 0.01 & 0.007 \\
& 2.35 & 0.75 & $0.834(32)$ & $0.05(1)$ & $1.14(29)$ & 0.09 & 0.03 \\
& 2.39 & 0.86 & $0.887(24)$ & $-0.001(8)$ & $1.47(22)$ & 0.05 & 0.02 \\
& 2.43 & 0.98 & $0.802(19)$ & $-0.011(7)$ & $0.85(19)$ & 0.008 & 0.003 \\
\hline \hline
\end{tabular}

Table 3.3.: Results for the fit parameters from fits with Eq. 3.8.

[16].

From the zero temperature string tension values in Tables. 3.1 and 3.2 one can conclude that the fit is only working at low temperatures.

We then tried two alternative fit ansätze. The first one, which is inspired by Eq. 3.2, is

$$
V(R, T)=V_{0}+\sigma(T) R+\gamma T \ln (2 R T)
$$

with three free parameters: the self energy $V_{0}$, a temperature dependent string tension $\sigma(T)$ and an arbitrary coefficient $\gamma$, and

$$
V(R, T)=V_{0}-\frac{\alpha}{R}+\sigma(T) R
$$

with also three free parameters: the self energy $V_{0}$, the coefficient $\alpha$ and a temperature dependent string tension $\sigma(T)$. The later one of these fit ansätze accounts for a $1 / R$ piece which reflects the Coulomb type behaviour for small $q \bar{q}$ separations and the first one accounts for a logarithmic behavior and both of them include the linear rising part in the potential. These two fits both work well as the data show either a Coulomb or a logarithmic behavior as well as the linearly rising feature. The results for the free parameters obtained from the fits with Eq. 3.8 and 3.9 are shown in Tables. 3.3 and 3.4 , respectively.

The fits have only been done on the $24 \times 12^{2} \times 6$ lattice. For all fits the minimum distance was $R_{\min } T=0.5$ which means $r=3 a$ in lattice units. Varying the minimum distance to be fitted does not lead to noticeable changes of the results.

In the Table. 3.3 the fits return values for the coefficient $\gamma$ of the logarithm which 


\begin{tabular}{|c|c|c|c|c|c|c|c|}
\hline$N_{\tau}$ & $\beta$ & $T / T_{c}$ & $a V_{0}$ & $a^{2} \sigma(T)$ & $\alpha$ & $\chi^{2}$ & $\left\langle\chi^{2}\right\rangle$ \\
\hline \hline \multirow{5}{*}{6} & 2.31 & 0.65 & $0.81(26)$ & $0.11(3)$ & $0.24(62)$ & 0.02 & 0.009 \\
& 2.35 & 0.75 & $0.91(5)$ & $0.069(6)$ & $0.41(11)$ & 0.3 & 0.1 \\
& 2.39 & 0.86 & $0.99(4)$ & $0.024(4)$ & $0.55(8)$ & 0.4 & 0.1 \\
& 2.43 & 0.98 & $0.87(3)$ & $0.004(4)$ & $0.32(8)$ & 0.07 & 0.02 \\
\hline \hline
\end{tabular}

Table 3.4.: Results for the fit parameters from fits with Eq. 3.9.

are approximately equal to 1 within an error margin. Fixing $\gamma$ to 0 leads to a decreasing string tension when $R_{\min }$ is raised. Thus, in order to account a purely linear rise at large distances as long as the minimum distance is fixed one can not exclude the logarithmic term or can not fix $\gamma$ to 0 .

The resulting string tension values in Table. 3.4 normalized to its zero temperature value are shown in Fig. 3.2.

We set the coefficient $\alpha$ of the Coulomb type term in Eq. 3.9 as a free parameter in the potential fits and the fits return values which are bigger but with large errors than $\pi / 12=0.26$ for the coefficient.

We see the decreasing string tension with rising temperature in Fig. 3.2. Recall that the string model prediction assumes a second order transition with a continuous vanishing of the string tension at the critical temperature (Eq. 3.3). Our results of $\sigma(T) / \sigma(0)$ in Fig. 3.2 confirm the prediction giving the value $0.07(7)$ at $T / T_{c}=0.98$.

The string tension $\sigma(T)$ is compared with the leading behavior $\sigma(0)-\pi T^{2} / 3$ as given in Eq. 3.2. The latter values are shown as the triangle points in Fig. 3.2. A clear deviation is observed between the line that connects the triangle points and $\sigma(T) / \sigma(0)$. This deviation reflects the thermal corrections of the temperature-dependent terms to the zero temperature string tension.

Above $T_{c}$, the potential is exponentially screened. Motivated by the decrease of the potential caused by the increase of temperature discussed above, it is natural to be interested in how the flux tube connecting quarks reacts when temperature changes. In 


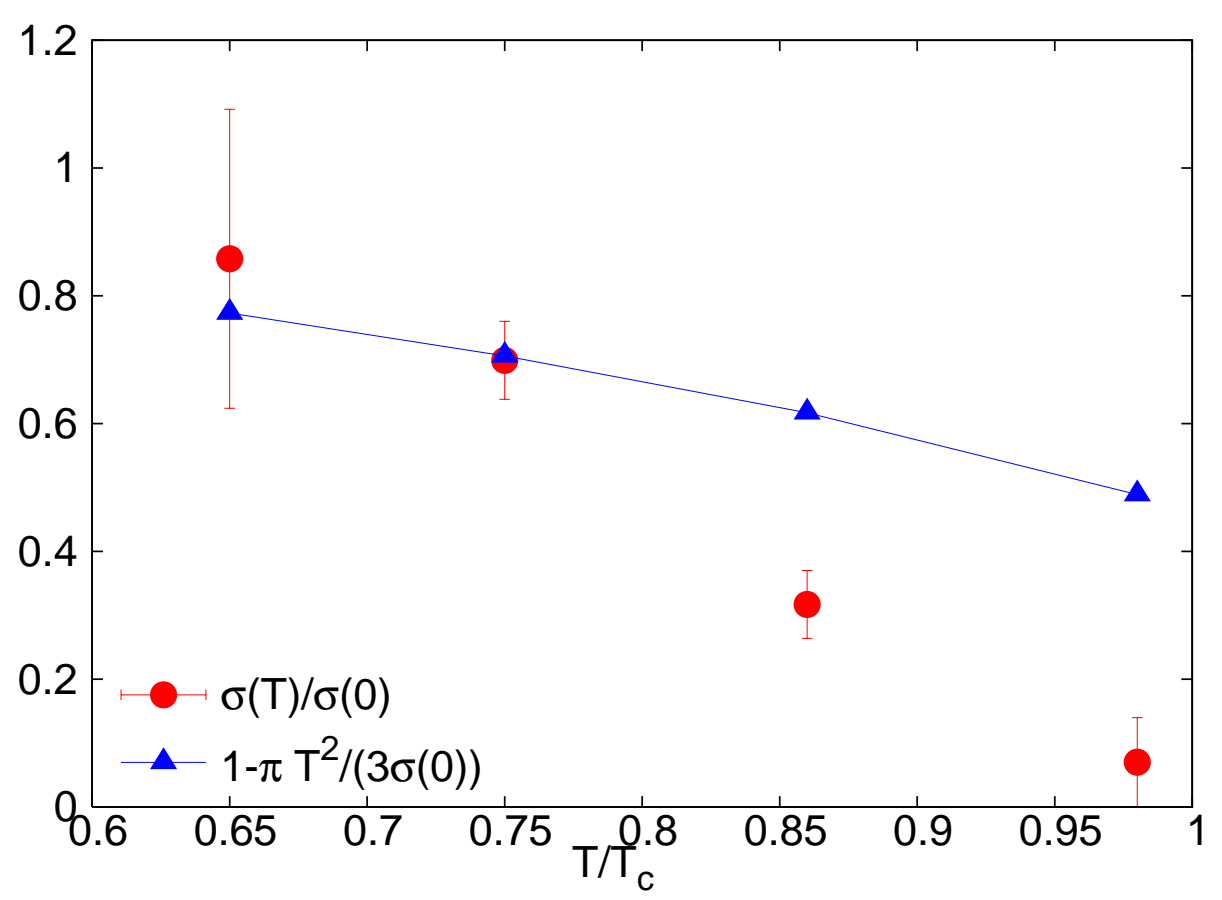

Figure 3.2.: The string tension as obtained from fits with Eq. 3.9, normalized to its zero temperature value. The data are compared with the lowest-order temperature effect on the linear part of the potential (Eq. 3.2), shown as the triangle points. 
particular, we are interested in $T$-dependence of the shape and size of the tube. The rest of this work is devoted to have answers to these questions. 


\section{Flux Tube Model}

In this chapter we will give a description of a model that becomes a central object to be explored in our simulation.

The color flux tube models were developed to describe the deconfinement phase transition. Ours is the case of a pure $S U(2)$ gauge theory. Flux tubes connect quarks and antiquarks on the lattice. The flux tube is characterized by two parameters: the string tension $\sigma$ and the width $D$. We assume that there is a constant energy per unit length along the tube. This is $\sigma$.

The linearly rising potential with the $q \bar{q}$ separation is what is expected from the flux tube picture of confinement.

In contrast to quantum electrodynamics (QED), where the field lines connecting a pair of opposite charges are allowed to spread, one expects that the quarks within a hadron are the sources of chromoelectric flux which is concentrated within narrow tubes connecting the constituents. Unlike the neutral photon of QED, gluons themselves participate in strong interactions. The gluon has the ability to do this as it carries color charge and so interacts with itself, making QCD significantly harder to analyse than QED. The flux tube picture of hadrons is depicted in Fig. 4.1.

Flux tubes can terminate only on quarks. A flux tube carries color indices at its ends. Gauge invariance implies that an allowed configuration of a quark and an antiquark on adjacent sites is the one in which the quark and an antiquark are linked by a string so that the colour index of quark (antiquark) and the colour index of the string at that end are contracted to form a colour singlet. When a quark and an antiquark are far apart, flux tubes have to be excited to connect the two sites. When there is enough energy available to create a new $q \bar{q}$, the system breaks up permitting the formation of two colour singlets. 


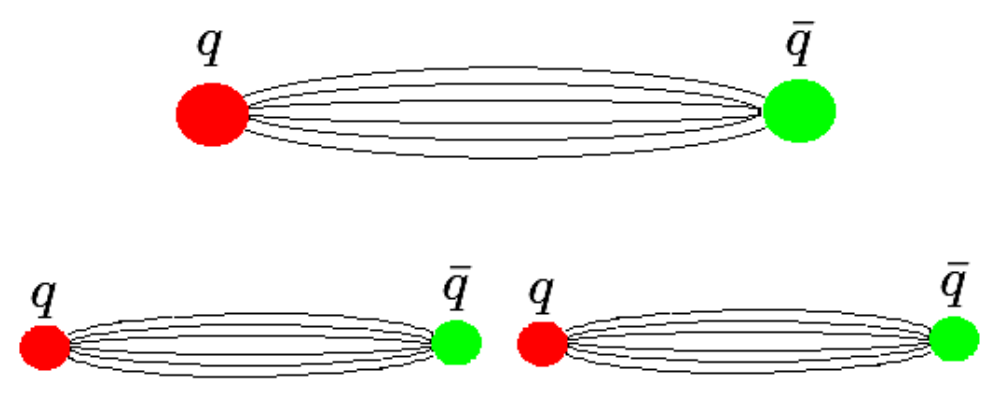

Figure 4.1.: Separation of a $q \bar{q}$ pair.

Since the static interquark potential describes the strong interaction in the region between static sources, the details of the interaction can be seen by calculating the chromoelectric and chromomagnetic field distributions in this narrow tube. It is therefore of great interest to study the spatial distribution of the energy density between two static sources in a pure gluonic sector. Then the investigation of the confining string converts to the scanning the nature of the flux tube. First calculations of the flux distribution as a function of $q \bar{q}$ separations have been carried out by Fukugita and Niuya [17], by Flower and Otto [18] for $S U(3)$ and by Sommer [1]. For the case of the $S U(2)$ gauge theory, quite detailed calculations of the flux distribution for $q \bar{q}$ separations up to six lattice units have been done by Haymaker et al. [2, 19].

\subsection{Dual Superconductor Picture of Confinement}

The most popular mechanism of colour confinement in QCD is dual superconductivity of the vacuum [20], idea of which based on that the solenoidal magnetic monopole currents that surround the flux tube leads to flux tube formation. It has been suggested that the QCD vacuum is a condensate of gluons and as well as light quark-antiquark pairs. This is somewhat analogues to the ground state of a superconductor. There the condensate of paired electrons gives rise to the Meissner effect of magnetic flux exclusion from the condensate unless the energy balance favours a local breakdown to the normal phase. And one can imagine placing a pair of magnetic monopole and antimonopole into this superconducting medium, the magnetic flux will be confined to a string-like configuration joining the pair of monopoles. 


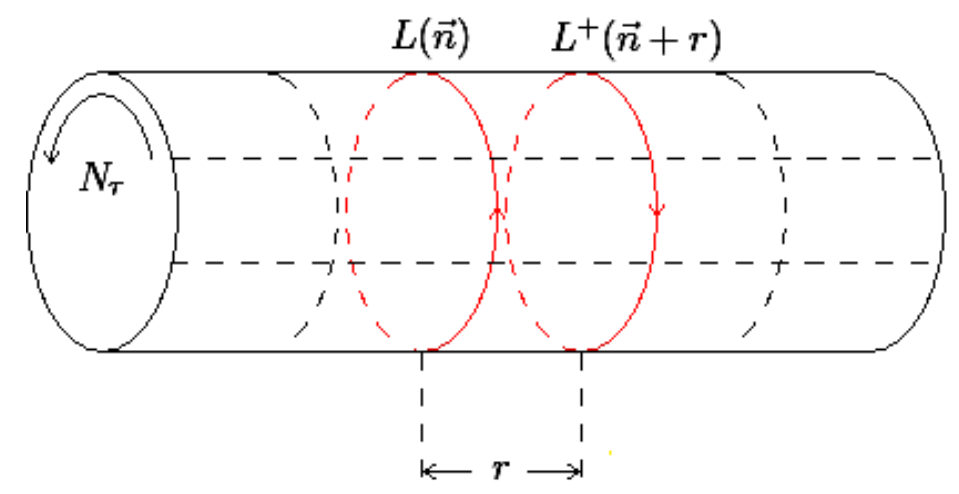

Figure 4.2.: Polyakov loops on the lattice.

Analogous to this situation the chromoelectric field in the region of space between a $q \bar{q}$ pair is constrained by dual Meissner effect into Abrikosov flux tubes, generating a static potential proportional to the interquark distance. In the zero temperature confined phase, some numerical results of the distribution of the colour flux around the quark sources support this conjecture $[2,3,19]$. From the standpoint of this theory, the flux distribution in the flux tube is explained by the two main effects that the abelian monopoles are expelled from the region of space between a quark pair creating monopole condensation and this causes the squeezing of the abelian electric flux into an almost one-dimensional flux tube. More details about the conjecture can be found in the literature $[2,3,19]$. Gross features of colour field distribution between quark sources are understood as a result of many constructive studies based on this theory, mainly focusing on the flux formation, flux tube distributions as a function of lattice volume and $q \bar{q}$ separations at zero temperature case $[3,19]$. We will just recall some of those results in this chapter.

\subsection{Lattice Measures of the Flux Tube}

The distribution of gluon fields in the flux tube is measured as follows. Time propagation of the two static quarks sitting in the ends of the flux tube are represented by Polyakov loop $L$ and its conjugate $L^{+}$, which are located at distance $r$ from each other on the lattice as shown in Fig.4.2. 


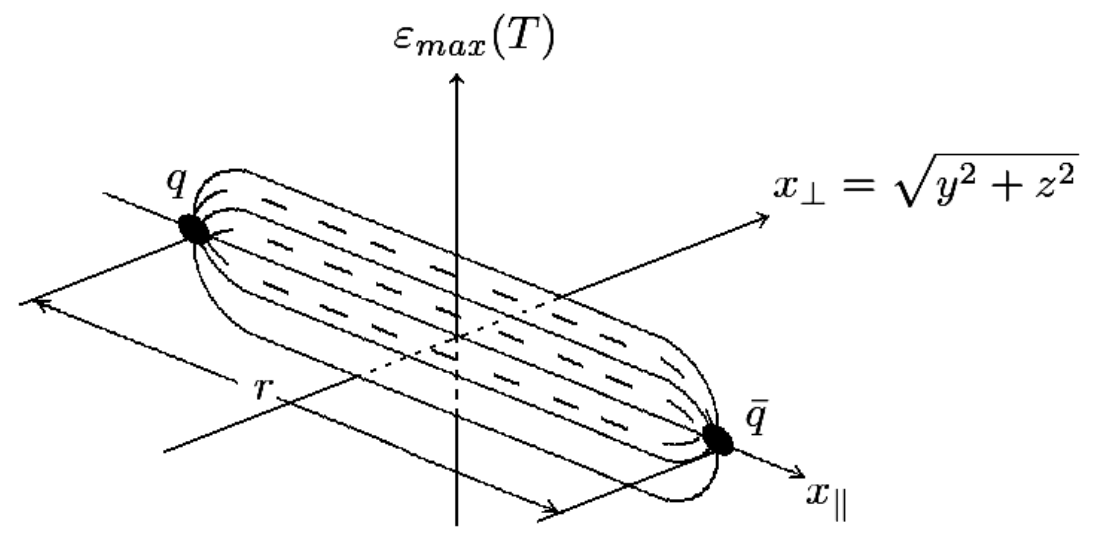

Figure 4.3.: Sketch of the geometry.

The plaquette variable

$$
\square_{\mu \nu}=\frac{1}{N_{c}} \operatorname{Tr}\left(U_{\mu, \nu}\right)
$$

with the orientation $(\mu, \nu)$, which has six different values $(2,3),(1,3),(1,2),(1,4)$, $(3,4),(2,4)$, measures the field strength. Flux tube profiles can thus be extracted from the correlation of a plaquette with the Polyakov loops

$$
f_{\mu \nu}(\mathbf{r}, \mathbf{x})=\frac{\beta}{a^{4}}\left[\frac{\left\langle L(0) L^{+}(\mathbf{r}) \square_{\mu \nu}(\mathbf{x})\right\rangle}{\left\langle L(0) L^{+}(\mathbf{r})\right\rangle}-\left\langle\square_{\mu \nu}\right\rangle\right] .
$$

by varying the distance $\mathbf{x}$ and the orientation of the plaquette with respect to the Polyakov loops. A visualisation of the flux tube in 3-space is depicted in Fig. 4.3, where one can easily read off the longitudinal $x_{\|}$as well as the transverse distances $x_{\perp}$ of the plaquette from the sources.

Six different $(\mu, \nu)$ combinations define the six components of electric and magnetic fields. Three space-space plaquettes correspond to the magnetic fields and three spacetime plaquettes correspond to the electric fields:

$$
\begin{aligned}
f_{12} & \rightarrow \frac{1}{2}\left(-B_{\perp}^{2}\right) & f_{24} & \rightarrow \frac{1}{2} E_{\perp}^{2} \\
f_{13} & \rightarrow \frac{1}{2}\left(-B_{\perp}^{2}\right) & f_{34} & \rightarrow \frac{1}{2} E_{\perp}^{2} \\
f_{23} & \rightarrow \frac{1}{2}\left(-B_{\|}^{2}\right) & f_{14} & \rightarrow \frac{1}{2} E_{\|}^{2}
\end{aligned}
$$


The directions of orientations of the field components (i.e orthogonal and parallel) describe their orientation to the axis connecting $q \bar{q}$ which we choose to be in the 1 direction. The derivation of the correspondence between the field strength tensor in Euclidean space and Minkowski space is treated in Appendix B.

Total magnetic and electric field strengths, respectively, are

$$
\begin{gathered}
\mathcal{M}=-\left(f_{12}+f_{13}+f_{23}\right) \\
\mathcal{E}=f_{24}+f_{34}+f_{14} .
\end{gathered}
$$

Combinations of $\mathcal{M}$ and $\mathcal{E}$ define the total energy and action density,

$$
\begin{aligned}
\varepsilon & =\mathcal{E}+\mathcal{M} \\
\delta & =\mathcal{E}-\mathcal{M}
\end{aligned}
$$

Since the magnetic contributions are negative, there is a cancellation between the two terms in the energy density, while the action density is amplified. We can thus expect that the action density will be much larger than the energy density.

\subsection{Flux Tube at Zero Temperature}

In this section we will briefly summarize what has been done so far about flux tube distributions at zero temperature. Some evidence of superconductivity of the vacuum has been produced by Monte Carlo numerical simulations on the lattice. Numerical studies of the distribution of the colour flux around the quark sources $[1,2,3]$ based on this conjecture confirm that in the confining phase the center width of the colour flux tube joining a pair of quarks [3]

$$
D_{\delta}^{2}=\frac{\int_{0}^{x_{c u t}} d x_{\perp} x_{\perp}^{3} \delta\left(r / 2, x_{\perp}\right)}{\int_{0}^{x_{c u t}} d x_{\perp} x_{\perp} \delta\left(r / 2, x_{\perp}\right)}
$$

is a physical quantity which is independent of the interquark distance and is fixed by the parameters of the Ginzburg-Landau formulation [21] of the standard superconductivity. Here $x_{\perp}$ and $x_{c u t}$ denote the transverse distance and the maximum transverse distance 
for which the measurements were performed, respectively. Full details of the lattice technique and implementation of the colour field operators can be found in $[3,4,19,22,23]$.

Most of these studies focused on the transverse profile of field distributions in the middle plane of the flux tube. The energy and action density profiles for $S U(2)$ that were obtained by Bali, Schilling and Schlichter [3], who have studied the distribution for $q \bar{q}$ separations up to about $2 \mathrm{fm}$, were consistent with those obtained by Haymaker et al. [4]. Bali et al. were mainly interested in studying the behavior of the energy and action densities with large $q \bar{q}$ separation, which can ascertain impact of the finite size of the lattice on the flux distribution results.

The basic parameters one is interested in are the maximum value $\varepsilon_{\max }$ of the energy density and widths $D$ of the energy and action density. Basic studies have been done on the behavior of the energy and action density distributions as functions of $q \bar{q}$ separation, concentrating on the question whether the maximum value of the field distribution of the energy density, $\varepsilon_{\max }$, goes to a constant or goes to zero with increasing $q \bar{q}$ separation, $r$ [4]. Speculating that the string tension $\sigma$ should be constant from the linearly confining potential they have given evidence that $\varepsilon_{\max }$ at $x_{\|}=r / 2$ and $x_{\perp}=0$ goes to a constant as a function of $r$. In [3] the $r$ dependence of the width of the energy and action density is displayed. Since the width reaches a constant value at large $q \bar{q}$ separation after some increasing, the maximum value of the field distribution must also reach a constant value after some decreasing.

The $r$-dependence of the flux distributions can also be clearly seen in [19], where the value of the action density on the middle plane between $q \bar{q}$ decreases first as $r$ increases and then it approaches a finite value as $r$ becomes large in the region $\beta<\beta_{c}$. Even at large $r$ the action density does not vanish. This means that string formation indeed occur in this phase as expected. What was also confirmed from the fit results [3] is that the width of the energy flux tube is smaller than the width of the action flux tube for distances below $0.5 \mathrm{fm}$ for the zero temperature case.

According to another conjecture [8, 9, 24, 25], which is the string picture of confinement, it is assumed that in the confining phase the mean squared width of the colour flux tube should grow logarithmically as a function of interquark distance. This calculation disagrees with the above introduced results based on the superconductivity picture, 
where the width stabilizes to a constant value at large $q \bar{q}$ separation. Interestingly, the width of the action density predicted by superconductivity theory alone was growing strongly with $r$ [1] as explained in the string theory. One does not know why the string model explanation should be relevant for the width of the action density and not for the width of the energy density of the flux tube.

All of the mentioned works, however, have demonstrated that by using Wilson loop plaquette correlations one can obtain much information on the flux tube problem on the required length scale of $1-2 \mathrm{fm}$. 


\section{Measurements on the Lattice}

\subsection{Update and Details of the Simulation}

Numerical method that is known as Monte Carlo method has been used successively in this work. In Monte Carlo simulation, some physical or mathematical system can be described in terms of probability distribution functions. In essence, the physics and mathematics are replaced by random sampling of possible states from probability distribution functions that describe the system. One can read the details about this method in $[26,27]$.

Wilson's lattice gauge theory with gauge group $S U(2)$ on a four dimensional simple hypercubic lattice with periodic boundary conditions has been used in the simulation. It is generally accepted that the $S U(3)$ gauge theory of quark and gluons describes the strong interactions. But because of the similarity of $S U(3)$ vacuum structure with the one of $S U(2)$ and also in order to simplify the simulation we had to restrict our analysis to the quenched approximation of $S U(2)$ pure gauge theories without matter fields. The standard Wilson action

$$
S_{G}(U)=\beta \sum_{n, \mu<\nu}\left[1-\frac{1}{2} \operatorname{Tr}\left(U_{n, \mu} U_{n+\mu, \nu} U_{n+\nu, \mu}^{+} U_{n, \nu}^{+}\right)\right] .
$$

is used in our simulation. Here the traces are of products of gauge links around the elementary plaquettes of the lattice.

Simulations have been carried out in the vicinity of the critical coupling values determined in Eq. 2.19 and 2.20. Instead of lattices with the same extension $N_{\sigma}$ in all 3 spatial directions we have used an extended number $N_{\|}$of lattice points in the direction of the $q \bar{q}$ axis. Our lattice sizes are thus $N_{\|} \times N_{\perp}^{2} \times N_{\tau}$ as illustrated in Fig. 5.1. 


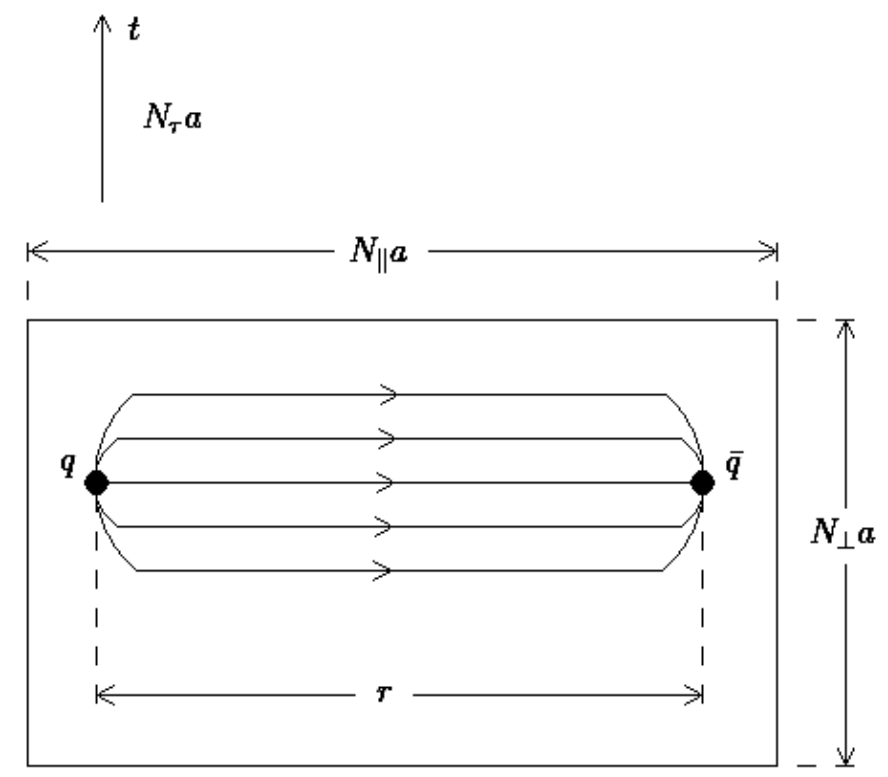

Figure 5.1.: Flux tube on the lattice.

The measurements are performed on lattices of size $N_{\|} \times N_{\perp}^{2} \times N_{\tau}$ with $N_{\tau}=4$ and 6 , with $N_{\|}=8,16$ and 24 and with $N_{\perp}=8$ and 12. Here $N_{\|}>N_{\perp}$ as it is illustrated in Fig. 5.1, due to our interest to have as large as possible $q \bar{q}$ separations along the $N_{\|}$ direction (see Fig. 5.1).

We used one heatbath update $[28,29,30]$ followed by four overrelaxation steps [31, 32] to obtain a new gauge configuration. Measurements of the observables were performed after each sweep. In order to thermalize the gauge configurations we allowed 2000 sweeps and then carried out between 20000 and 40000 measurements. As we could do the noise reduction by the link integration method [33] on the lattice of size $24 \times 12^{2} \times 6,20000$ measurements were done on this lattice reducing some computing time, while 40000 on the smaller lattices.

The electric and magnetic components described in Eq. 4.3 have been measured up to the transverse lattice distances $x_{\perp}=3,3$ and 4 for the three lattices $8^{3} \times 4,16 \times 8^{2} \times 4$ and $24 \times 12^{2} \times 6$ respectively and along the entire $q \bar{q}$ axis which means up to the longitudinal distance $x_{\|}=8$ for the first two lattices, while 12 for the largest lattice.

Numerical results have been obtained for the simulation parameters, i.e. the lattice 
coupling $\beta$, the temperature $T / T_{c}$, the lattice spacing $a$ and the number of configurations used at each $\beta$ value, given in Table. 5.1.

One can estimate that many symmetric values for the correlation of a plaquette with the two Polyakov loops will be produced over the lattice by the measurements due to the geometrically symmetric positions of the plaquette relative to the axis connecting $q \bar{q}$ as well as the middle plane between the $q \bar{q}$ pair. Therefore, we have averaged over the measurements on the symmetric lattice points. They have afterwards been duplicated again when we depict surface plots of the distributions.

\subsection{Error Reduction}

In this section we will shortly discuss two methods of noise reduction we used.

\subsubsection{The Reference Point Method}

It was found that the fluctuations are large in the quantity $L(0) L^{+}(\mathbf{r}) \square$. A significant improvement is achieved by replacing $\left\langle\square_{\mu \nu}\right\rangle$ with $\square_{\mu \nu}\left(\mathbf{x}_{R}\right)$ where $\mathbf{x}_{R}$ is a reference point placed far from the quark sources [19]. A corner of the lattice has been chosen as the reference point. This replacement has no effect on the measured average value due to the cluster decomposition theorem, but the statistical errors are significantly reduced. Thus Eq. 4.2 will be replaced by

$$
f_{\mu \nu}(\mathbf{r}, \mathbf{x})=\frac{\beta}{a^{4}}\left[\frac{\left\langle L(0) L^{+}(\mathbf{r}) \square_{\mu \nu}(\mathbf{x})\right\rangle-\left\langle L(0) L^{+}(\mathbf{r}) \square_{\mu \nu}\left(\mathbf{x}_{R}\right)\right\rangle}{\left\langle L(0) L^{+}(\mathbf{r})\right\rangle}\right] .
$$

First, the difference of two correlation terms, one of which is the Polyakov loop-plaquette correlation term and another one is the Polyakov loop-reference point correlation term, is calculated configuration by configuration instead of subtracting them after averages of those terms are computed. The reason for doing this is that the statistical errors of the terms will be added if we subtract the average of those correlation terms, while large amount of the errors will be cancelled during the subtraction configuration by configuration. 


\begin{tabular}{|c|c|c|c|c|}
\hline$N_{\|} \times N_{\perp}^{2} \times N_{\tau}$ & $\beta$ & $T / T_{c}$ & $a[\mathrm{fm}]$ & $N_{\text {meas }}$ \\
\hline \hline \multirow{5}{*}{$8 \times 8^{2} \times 4$} & 2.20 & 0.79 & 0.21 & 40000 \\
& 2.22 & 0.82 & 0.20 & 40000 \\
& 2.24 & 0.85 & 0.19 & 40000 \\
& 2.25 & 0.87 & 0.19 & 40000 \\
& 2.26 & 0.89 & 0.18 & 40000 \\
& 2.28 & 0.95 & 0.17 & 40000 \\
& 2.30 & 1.00 & 0.16 & 40000 \\
& 2.40 & 1.40 & 0.12 & 40000 \\
& 2.50 & 1.96 & 0.08 & 40000 \\
\hline \hline \multirow{5}{*}{$24 \times 8^{2} \times 4$} & 2.20 & 0.79 & 0.21 & 40000 \\
& 2.25 & 0.87 & 0.19 & 40000 \\
& 2.27 & 0.92 & 0.18 & 40000 \\
& 2.29 & 0.97 & 0.17 & 40000 \\
& 2.30 & 1.00 & 0.16 & 40000 \\
& 2.32 & 1.07 & 0.15 & 40000 \\
\hline \hline & 2.31 & 0.65 & 0.16 & 18000 \\
& 2.35 & 0.75 & 0.14 & 18000 \\
& 2.39 & 0.86 & 0.12 & 18000 \\
& 2.43 & 0.98 & 0.11 & 18000 \\
& 2.47 & 1.13 & 0.09 & 18000 \\
& 2.51 & 1.29 & 0.08 & 18000 \\
\hline \hline
\end{tabular}

Table 5.1.: Simulation parameters. 


\subsubsection{Noise Reduction by Link Integration}

The signal in the correlations between a plaquette and Polyakov loops also tends to get drowned in the statistical noise when the distance between two Polyakov loops increases. Here we used our second noise reduction technique [33] that is achieved by integrating out the temporal links in the Polyakov loops. The method is given in Appendix $\mathrm{C}$ in more detail and the achievement of using the method is going to be discussed in this subsection.

The trick can be used for measuring the correlation of two mutually independent variables, in our case two Polyakov loops. If the interaction is nearest neighbour, this requires that the two Polyakov loops are at least at distance of two lattice spacings. Additionally, to obtain electric or magnetic field strengths at a given lattice point we average four plaquettes in our algorithm, in order to arrive at operator insertions that are symmetric in respect to a given lattice site $\mathbf{n}$. Thus, finally in order to use the link integration method, $q \bar{q}$ separations must be at least four in lattice units which was two in lattice units in its original formulation of the link integration method. This forces us to use the method only at lattice $q \bar{q}$ separations $4 a, 5 a, 6 a, 7 a$ and $8 a$, not at $1 a, 2 a$ and $3 a$.

Here we are going to show the improvement of our lattice data by comparing nonlink integrated results with link integrated results. We present here only the parallel electric field strength, which is the largest one, and the parallel magnetic field strength representing the other 3 field strengths, which are equal approximately as we will see later in Chapter 6.

For $1 / 2 E_{\|}^{2}\left(x_{\|}, x_{\perp}\right)$ the comparison is displayed in Figs. 5.2 and 5.3 for the longitudinal and the transverse profiles respectively and for $-1 / 2 B_{\|}^{2}\left(x_{\|}, x_{\perp}\right)$ the same is done in Figs. 5.4 and 5.5. The compared data value and the plaquette distance are in units of the string tension but the $q \bar{q}$ separation is in lattice units on these figures.

The comparison is done at two values of $q \bar{q}$ separation, $r=4 a$ and $6 a$. At each value of the separations the data have been plotted for temperatures both below and above the critical temperature, which are $T=0.75 T_{c}$ and $T=1.13 T_{c}$.

The blue points are the link integrated results while the red square ones are obtained 

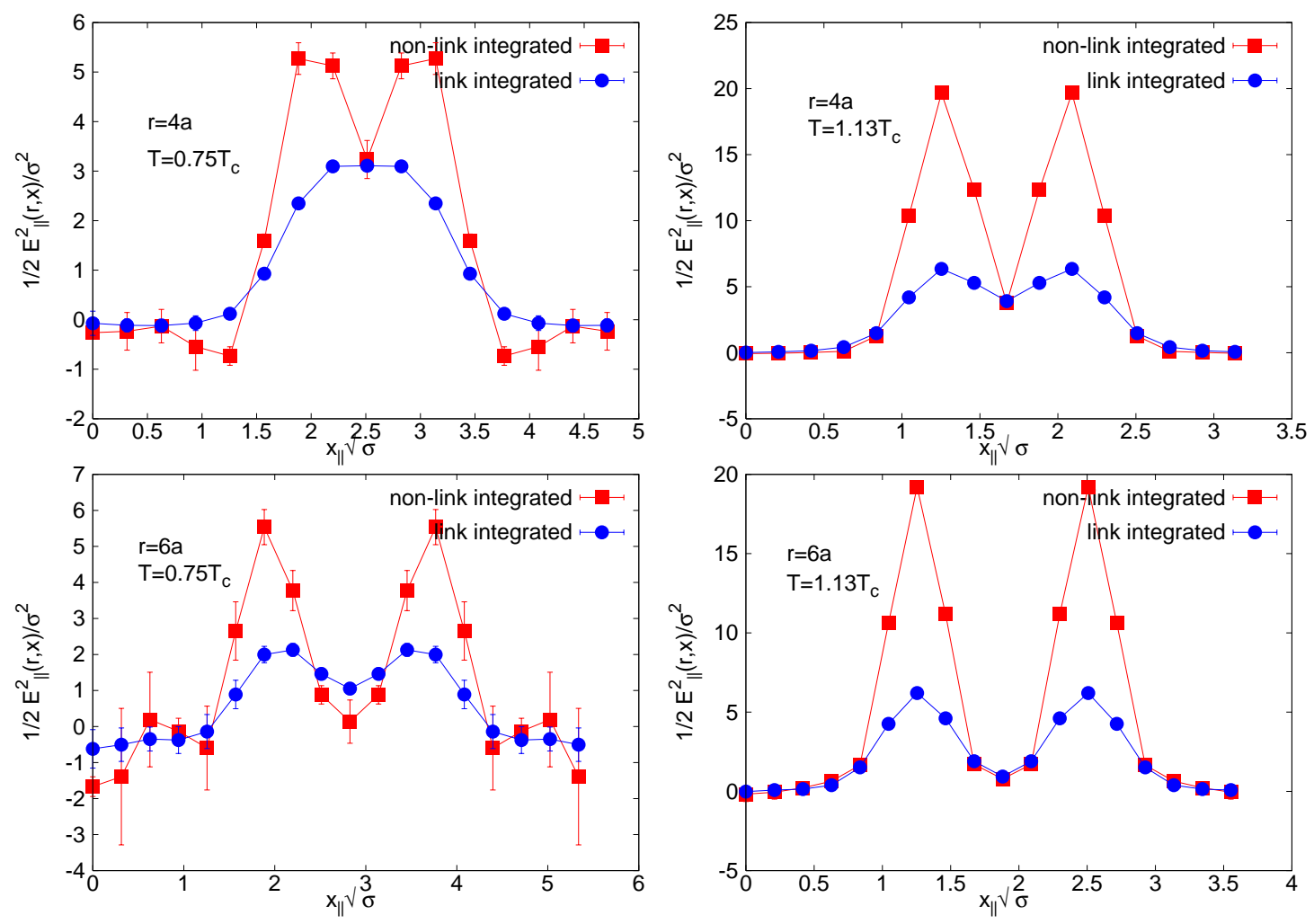

Figure 5.2.: Comparison of link integrated $1 / 2 E_{\|}^{2}\left(x_{\|}, x_{\perp}=0\right)$ with non-link integrated one. Lattice size is $24 \times 12^{2} \times 6$. Left: $r=4 a$ and $6 a$ at $T=0.75 T_{c} ;$ Right: $r=4 a$ and $6 a$ at $T=1.13 T_{c}$. 

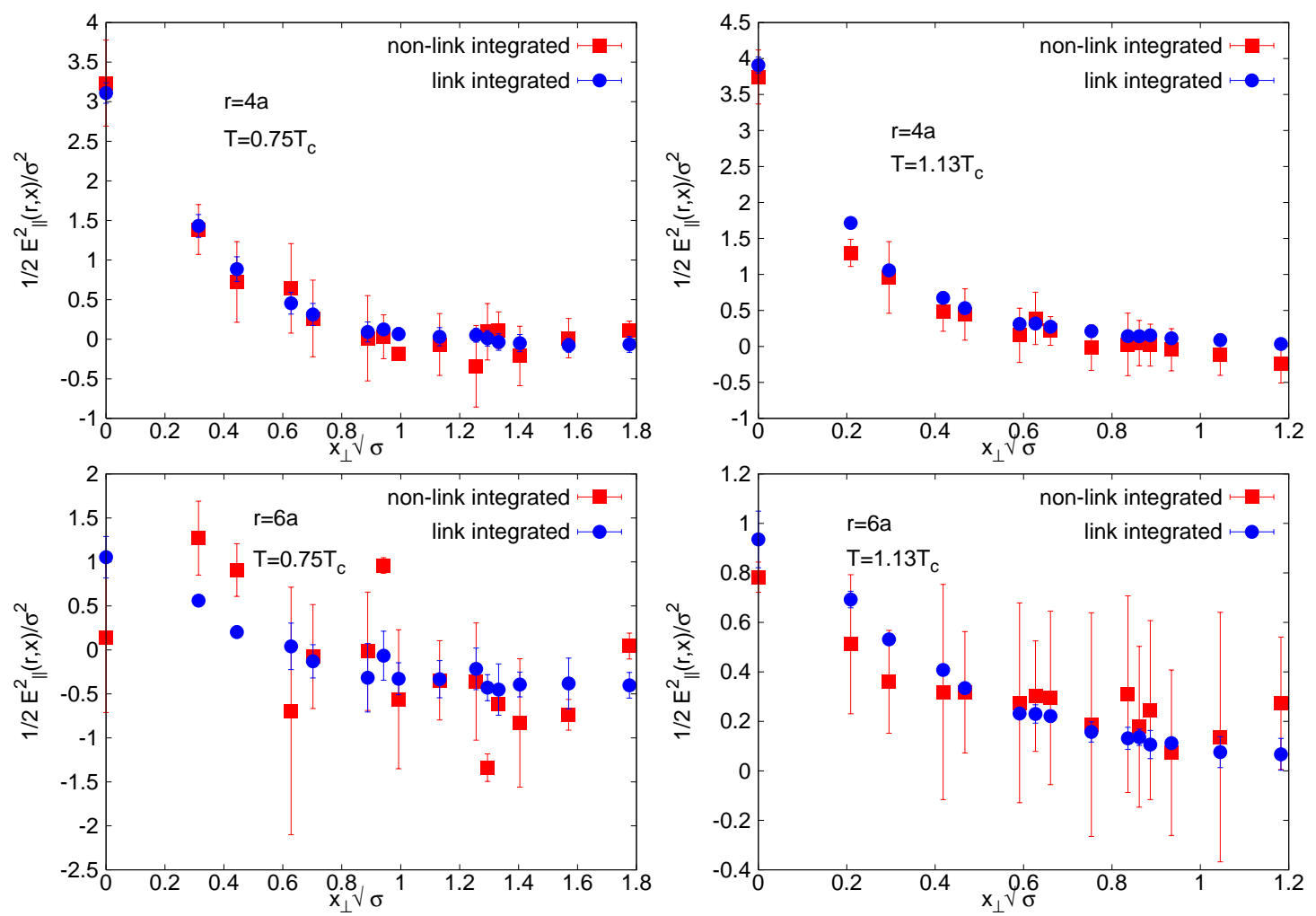

Figure 5.3.: Comparison of link integrated $1 / 2 E_{\|}^{2}\left(x_{\|}=r / 2, x_{\perp}\right)$ with non-link integrated one. Lattice size is $24 \times 12^{2} \times 6$. Left: $r=4 a$ and $6 a$ at $T=0.75 T_{c}$; Right: $r=4 a$ and $6 a$ at $T=1.13 T_{c}$. 

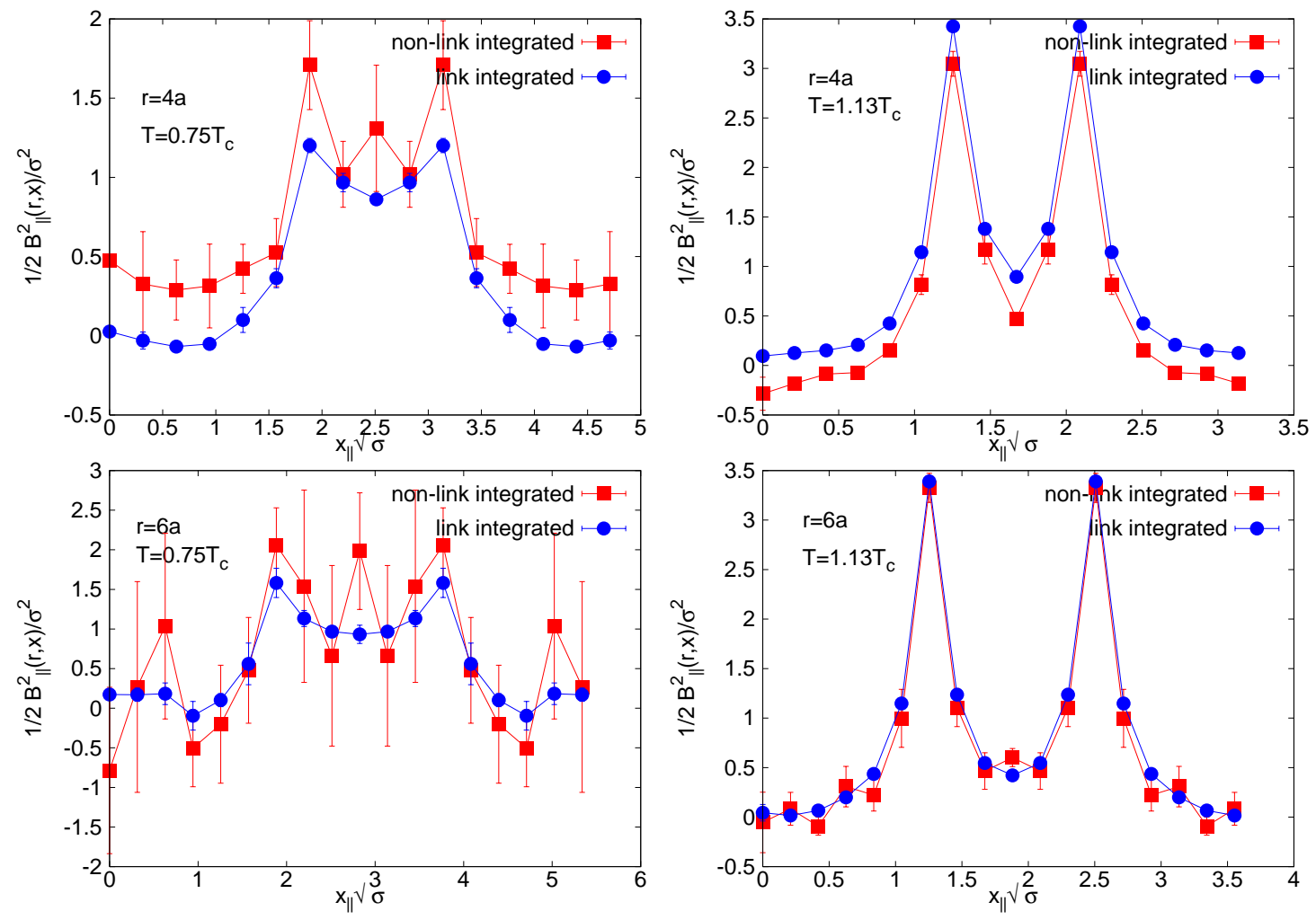

Figure 5.4.: Comparison of link integrated $-1 / 2 B_{\|}^{2}\left(x_{\|}, x_{\perp}=0\right)$ with non-link integrated one. Lattice size is $24 \times 12^{2} \times 6$. Left: $r=4 a$ and $6 a$ at $T=0.75 T_{c} ;$ Right: $r=4 a$ and $6 a$ at $T=1.13 T_{c}$. 

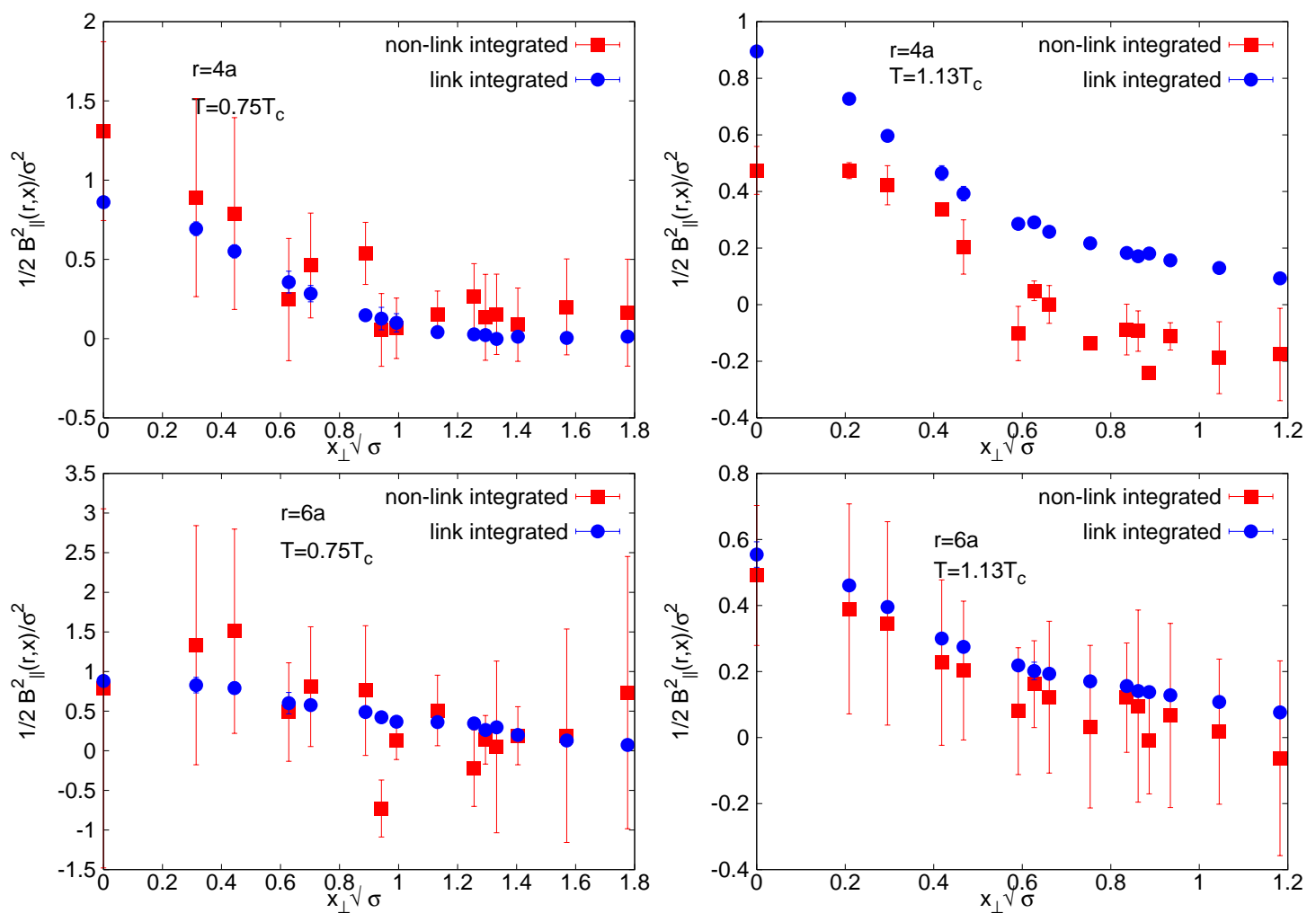

Figure 5.5.: Comparison of link integrated $-1 / 2 B_{\|}^{2}\left(x_{\|}=r / 2, x_{\perp}\right)$ with non-link integrated one. Lattice size is $24 \times 12^{2} \times 6$. Left: $r=4 a$ and $6 a$ at $T=0.75 T_{c}$;

Right: $r=4 a$ and $6 a$ at $T=1.13 T_{c}$. 
from the non-link integrated simulation. Let us see the difference between them first on the longitudinal profiles. Since the link integration has been performed only on the temporal links of the Polyakov loops, the link integrated and non-link integrated field strength values can be compared for plaquette-Polyakov loop distances larger than 2 lattice units. For the two nearest neighbour sites of the Polyakov loops as well as on top of the Polyakov loops the link integrated results can not be used, but are shown for completeness. However, since we are mostly interested in the flux tube at some large enough $q \bar{q}$ separation, the middle region between the $q \bar{q}$ pair where our analysis has been done is always far from the sources.

The non-link integrated field strength values in the two outside edges of the flux tube were fluctuating, sometimes having even negative values around zero. The error bars were also large. As a result of the link integration they have been stabilized around zero and look smoother. The error bars have become much smaller than the ones of the non-link integrated results. The same things happen to the transverse profiles.

Additionally, the fluctuation and the error bars of the results at temperatures above $T_{c}$ are always smaller than those of the results at temperatures below $T_{c}$ as we approach to the continuum with rising temperature. It can be seen comparing the plots in the left hand side with the ones in the right hand side.

The improvement achieved by the link integration tends to be smaller at smaller lattice spacings. This is due to the fact that the physical extent of the neighbourhood to be integrated out becomes smaller. On the other hand, the error of a non-link integrated operator also decreases with the lattice spacing. At the bottom line, the two effects almost cancel each other and the relative errors of link integrated Polyakov loops appear to remain rather independent of the lattice spacing, provided that the physical lattice volumes and the number of measurements are kept constant [3].

The application of the link integration significantly reduces computer time, because in order to get the same accuracy on the average values without the link integration, the simulation would require a larger number of measurements than the one with the link integration. 


\subsection{From the Lattice to the Continuum}

The lattice regularization has a temporary role only. At the end, regularization should be removed and the final predictions will not depend upon the specific regularization used. In a regularization, this process is done using a scaling relation. The lattice results come out in units of the lattice spacing $a$. The physical value of the lattice cutoff $a$ has to be fixed by assigning some dimensionful physical quantity to its experimental value, and afterwards the results can be expressed in physical units. Since this limit, where the results are in physical units is continuum, the functional dependence between the lattice spacing and the coupling can be computed perturbatively. This can be written as

$$
a \frac{d g}{d a}=b_{0} g^{3}+b_{1} g^{5}+\mathcal{O}\left(g^{7}\right)
$$

where the first two coefficients

$$
\begin{gathered}
b_{0}=\frac{1}{16 \pi^{2}}\left(\frac{11 N_{c}}{3}-\frac{2 n_{f}}{3}\right), \\
b_{1}=\left(\frac{1}{16 \pi^{2}}\right)^{2}\left(\frac{34 N_{c}^{2}}{3}-\left(\frac{10 N_{c}}{3}+\frac{N_{c}^{2}-1}{N_{c}}\right) n_{f}\right),
\end{gathered}
$$

are the same for the lattice discretisation and for the continuum QCD. After the integration the functional dependence between the lattice spacing and the coupling becomes

$$
a \Lambda_{L} \equiv R(\beta)=\left(\frac{2 N_{c} b_{0}}{\beta}\right)^{\frac{-b_{1}}{2 b_{0}^{2}}} \exp \left\{-\frac{\beta}{4 N_{c} b_{0}}\right\} \text {. }
$$

Here $\Lambda_{L}$ is the so-called $\Lambda$ parameter of the theory. This parameter sets the scale for QCD. It is an external parameter, like the fine structure coupling $\alpha \approx 1 / 137$ in QED. If lattice results at finite $a$ show the behavior of the right hand side of the $a \Lambda_{L}$, a reliable continuum extrapolation is possible.

For the determination of our scaling function we used the scaling relation based on the $S U(2)$ zero temperature string tension results given by [16].

The string tension $a \sqrt{\sigma}$ can be expressed as

$$
a \sqrt{\sigma}=\frac{\sqrt{\sigma}}{\Lambda_{L}} R(\beta)
$$




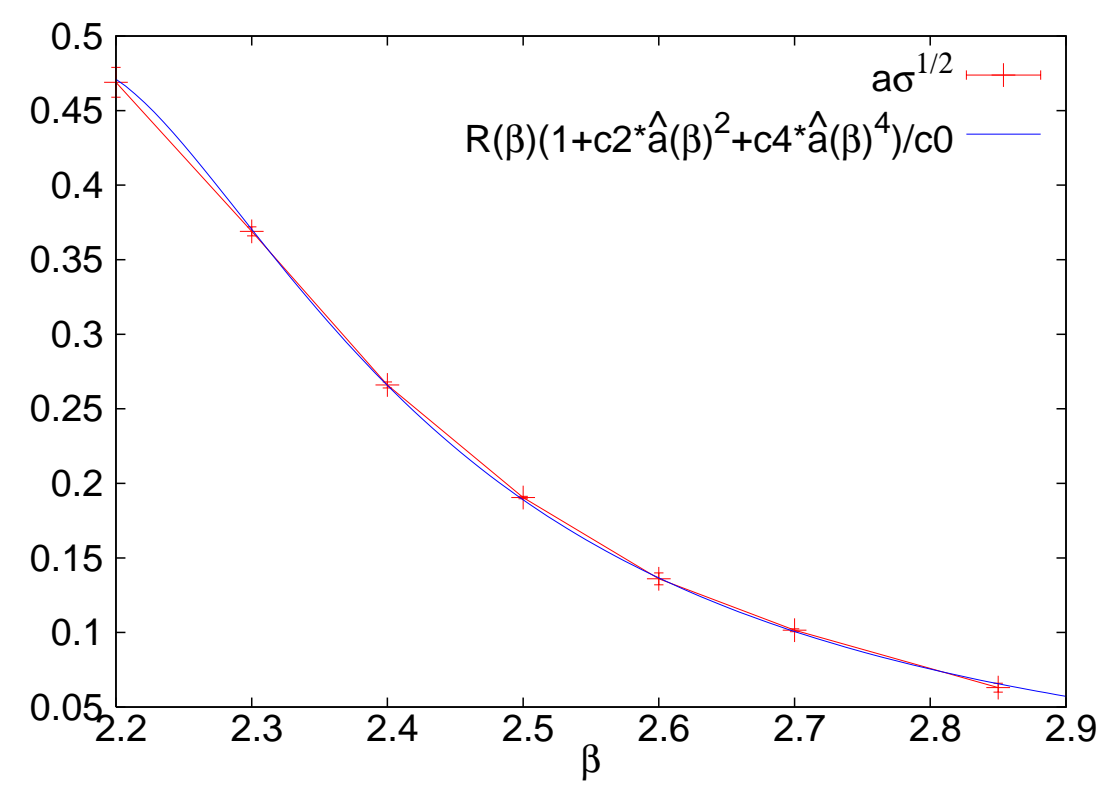

Figure 5.6.: Fit of $a \sqrt{\sigma}$ data with the continuum. The red line connects the data and the blue line is the fit to Eq. 5.6.

with $R(\beta)$ as given in Eq. 5.4. But Eq. 5.5 will not describe our data. The reason for this is that the left hand side of the equation is the lattice data, while $R(\beta)$ in the right hand side is perturbative, therefore it is only valid at small $g^{2}$ or large $\beta$. To fit the data for $a \sqrt{\sigma}$ we therefore use a renormalisation group inspired ansatz

$$
\sqrt{\sigma} a(\beta)=R(\beta)\left(1+c_{2} \hat{a}^{2}(\beta)+c_{4} \hat{a}^{4}(\beta)\right) / c_{0}=F(\beta)
$$

with $\hat{a} \equiv R(\beta) / R(\bar{\beta})$. Here $\bar{\beta}=2.70$ is the reference point which was chosen as the $\beta$ value that has the smallest error bar from our data.

The fit is shown in Fig. 5.6, where the dotted line connects the $a \sqrt{\sigma}$ data which is the left hand side of Eq. 5.6 and the solid line is the fit results.

The fit was done in the interval $[2.20: 2.90]$ of $\beta$ and the fit parameters were found to be

$$
\begin{gathered}
c_{0}=0.0234958 \pm 0.0002972, \\
c_{2}=0.10258 \pm 0.005234, \\
c_{4}=-0.00534346 \pm 0.00032 .
\end{gathered}
$$

Now our scaling function $F(\beta)=a(\beta) \sqrt{\sigma}$ has been completely determined. So we can obtain estimates of the corresponding temperatures in units of the critical temperature 
at each $\beta$

$$
\frac{T}{T_{c}}=\frac{F\left(\beta_{c}\right)}{F(\beta)}
$$

Estimates of the physical values of the lattice spacings at each $\beta$ have been obtained by setting $\sqrt{\sigma}=440 \mathrm{MeV}$. They are displayed in Table. 5.1.

The lattice spacing $a(\beta)$ has a different value for each $\beta$. This means that a $q \bar{q}$ separation value that was equal in lattice units will give a different value in physical units because $r_{\text {phys }}=r_{\text {lat }} a(\beta)$. Since we want to see the temperature dependence of the flux distribution at a fixed value of the physical $q \bar{q}$ separation $r_{p h y s}$, we have to collect the data at approximately matching physical $q \bar{q}$ separations but for various temperatures. The physical values of the $q \bar{q}$ separations which we could study here are summarized in Tables. 6.1 and 6.2 . 


\section{Analysis for Flux Tube Profiles at Finite Temperature}

Now we are in the position to do a detailed analysis of the results we have obtained. We have studied correlations of a plaquette with Polyakov loops describing the two-quark static-meson system as defined in Eq. 4.2.

Recall the six definitions given by Eq. 4.3. We have studied the individual parallel and orthogonal contributions of the chromoelectric and chromomagnetic field strengths to the energy and action densities of the flux tube in the vicinity of $T_{c}$.

\subsection{Distribution Surface}

Distribution surfaces of $1 / 2 E_{\|}^{2}(r, \mathbf{x}), 1 / 2 E_{\perp}^{2}(r, \mathbf{x}), 1 / 2 B_{\|}^{2}(r, \mathbf{x})$ and $1 / 2 B_{\perp}^{2}(r, \mathbf{x})$, in units of the string tension, are shown in Figs. 6.1, 6.2, 6.3 and 6.4 at some values of the physical $q \bar{q}$ separation and temperature. The results on Figs. 6.1 and 6.2 have been obtained from the $16 \times 8^{2} \times 4$ lattice, while the results on Figs. 6.3 and 6.4 come from the $24 \times 12^{2} \times 6$ lattice.

The distribution surfaces show the general behavior that any field is distributed all over the space in such a way that it has a peak on $q$ and $\bar{q}$ and its value decreases as the point on which the field strength is computed goes away from the $q$ or $\bar{q}$. In general, the distributions look geometrically symmetric relative to the middle plane perpendicular to the $q \bar{q}$ axis at $x_{\|}=r / 2$.

The data have relatively large fluctuations for small values of $\beta$ as is clearly visible in the figures of the distribution surfaces. As $\beta$ increases it approaches to the continuum 

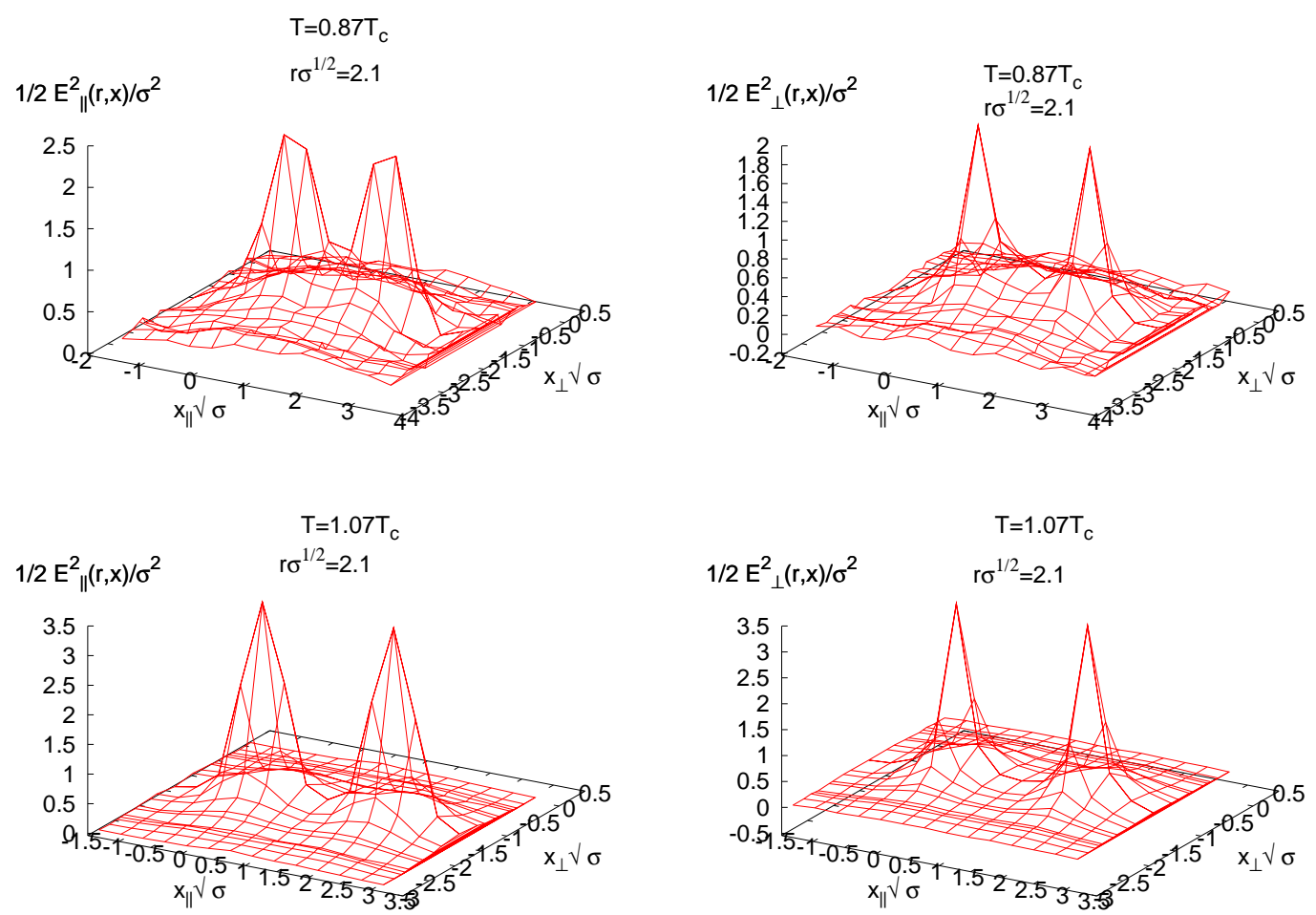

Figure 6.1.: Left: Distribution of $1 / 2 E_{\|}^{2}\left(x_{\|}, x_{\perp}\right)$ and Right: Distribution of $1 / 2 E_{\perp}^{2}\left(x_{\|}, x_{\perp}\right)$ at $r \sqrt{\sigma}=2.1$ for $T=0.87 T_{c}$ and $1.07 T_{c}$ from $16 \times 8^{2} \times 4$ lattice. 

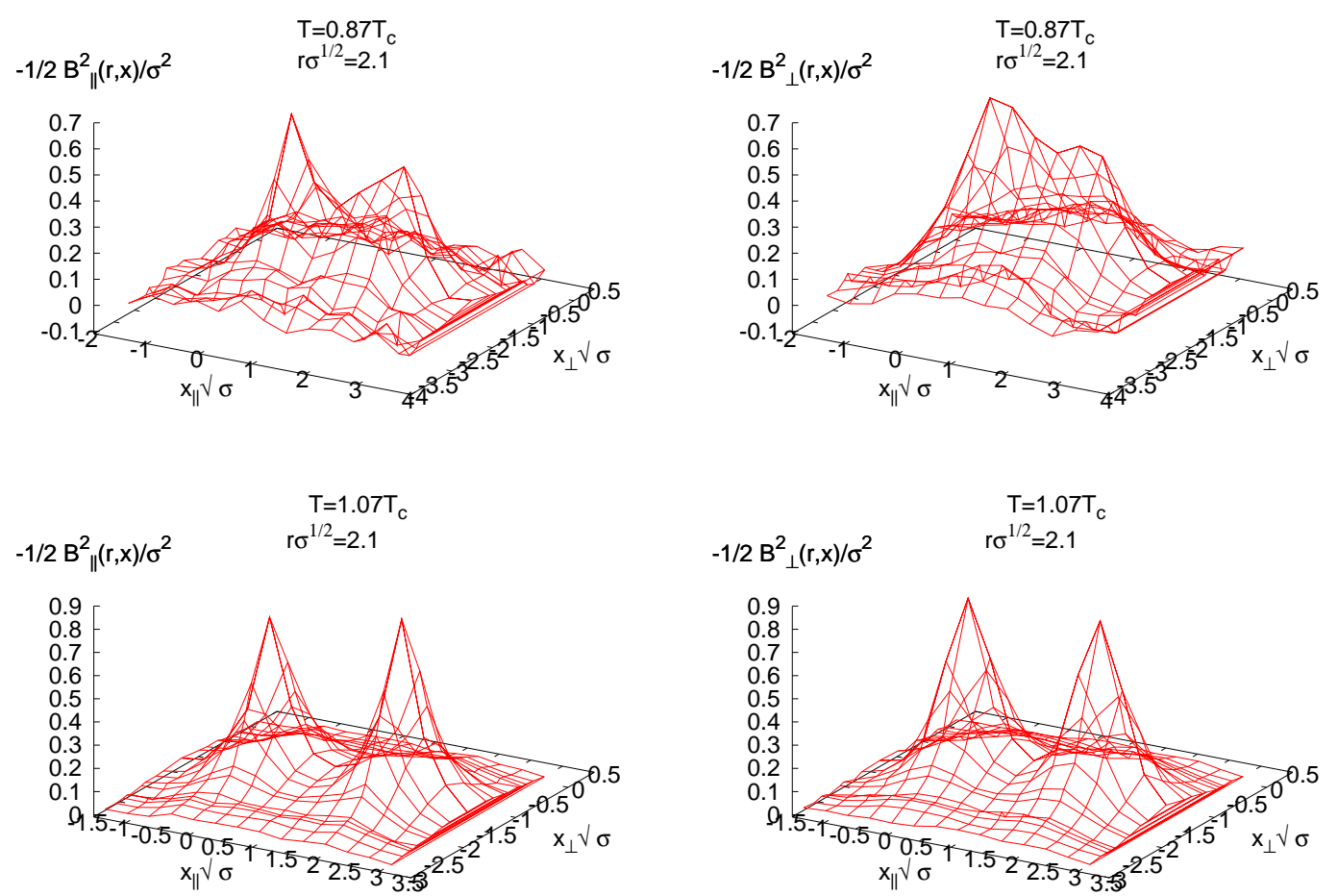

Figure 6.2.: Left: Distribution of $1 / 2 B_{\|}^{2}\left(x_{\|}, x_{\perp}\right)$ and Right: Distribution of $1 / 2 B_{\perp}^{2}\left(x_{\|}, x_{\perp}\right)$ at $r \sqrt{\sigma}=2.1$ for $T=0.87 T_{c}$ and $1.07 T_{c}$ from $16 \times 8^{2} \times 4$ lattice. 

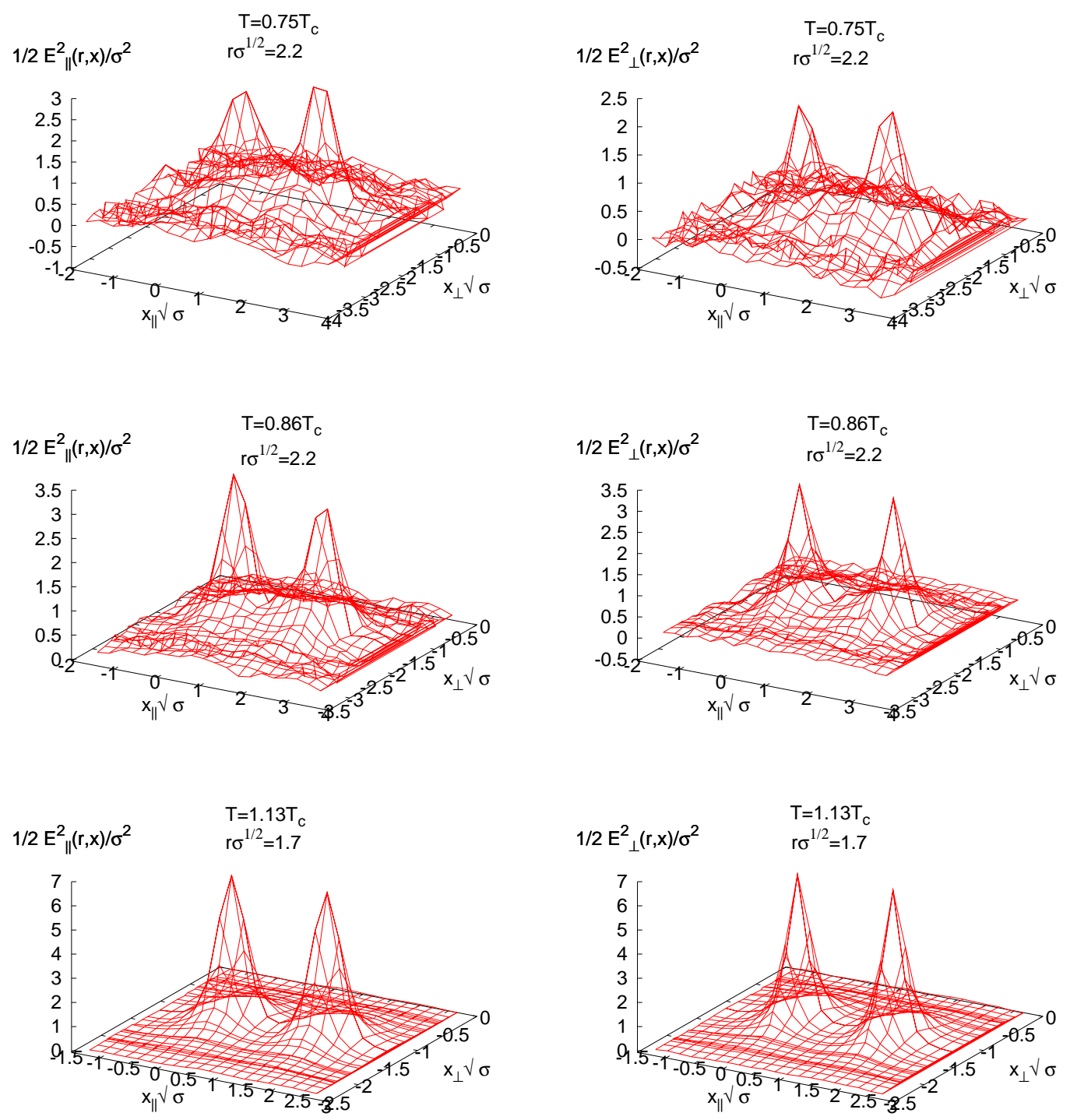

Figure 6.3.: Left: Distribution of $1 / 2 E_{\|}^{2}\left(x_{\|}, x_{\perp}\right)$ and Right: Distribution of $1 / 2 E_{\perp}^{2}\left(x_{\|}, x_{\perp}\right)$ at $r \sqrt{\sigma}=2.2$ and 1.7 for $0.75 T_{c}, 0.86 T_{c}$ and $1.13 T_{c}$ from $24 \times 12^{2} \times 6$ lattice. 

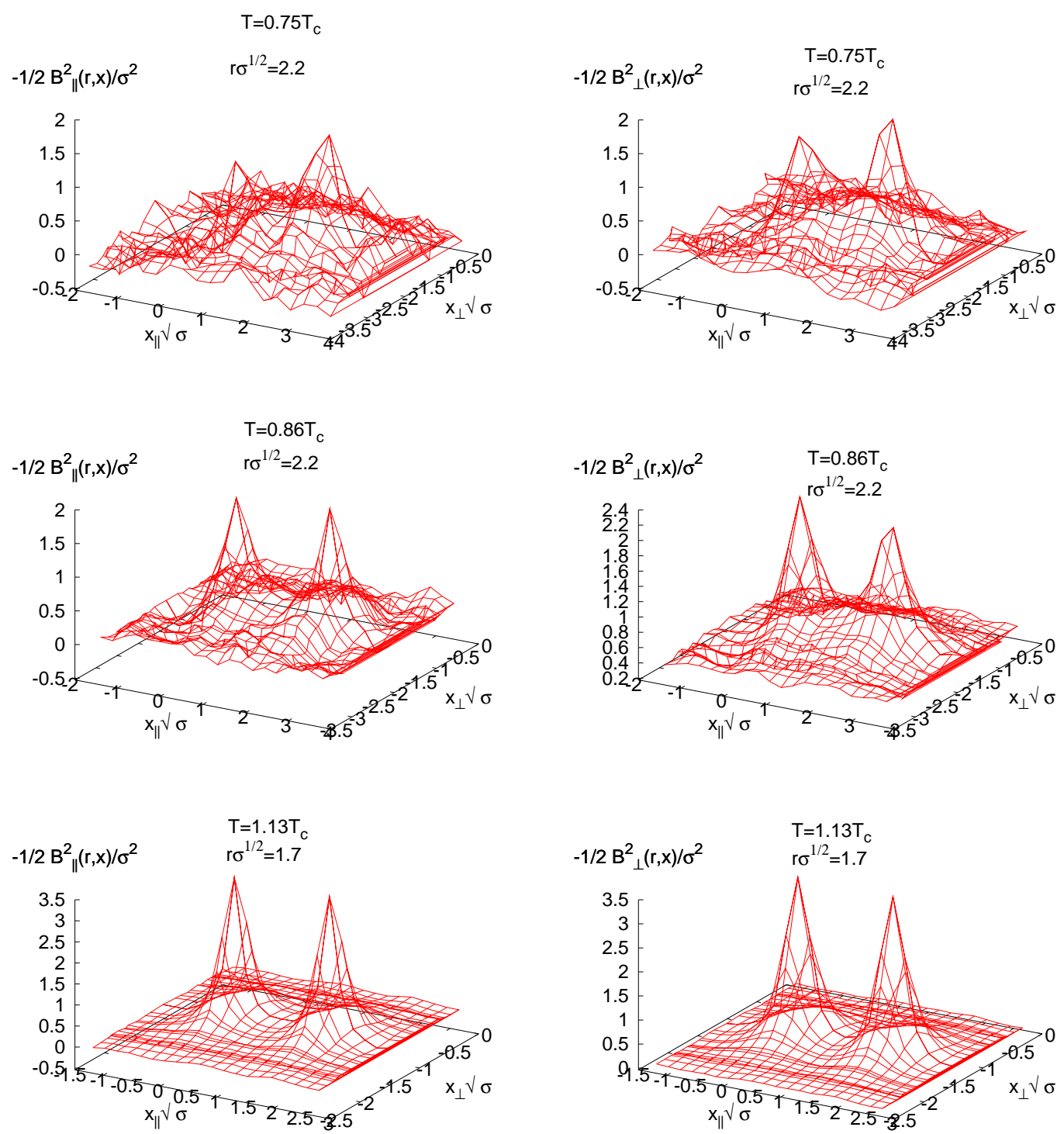

Figure 6.4.: Left: Distribution of $1 / 2 B_{\|}^{2}\left(x_{\|}, x_{\perp}\right)$ and Right: Distribution of $1 / 2 B_{\perp}^{2}\left(x_{\|}, x_{\perp}\right)$ at $r \sqrt{\sigma}=2.2$ and 1.7 for $0.75 T_{c}, 0.86 T_{c}$ and $1.13 T_{c}$ from a $24 \times 12^{2} \times 6$ lattice. 
and the distribution surfaces are becoming smooth. Therefore it is getting more symmetrized when the temperature increased. We avoid use of an interpolating procedure prefering to judge the significance of the actual data.

On the distribution surfaces both a longitudinal $\left(x_{\|}\right)$and a transverse $\left(x_{\perp}\right)$ distribution of any field strength is easily seen at the same time. In the following we will look at the longitudinal and the transverse profiles of these distribution surfaces separately in detail.

\subsection{Longitudinal Profiles}

A longitudinal profile of a field strength shows how the field strength value is spread along the direction parallel to the axis connecting $q \bar{q}$ pair at a fixed constant $x_{\perp}$. Throughout the work we focused on the longitudinal profiles at $x_{\perp}=0$ since the plane, $\left(x_{\|}, x_{\perp}=0\right)$, on which the quark sources are both placed has the maximum field strength value which apparently decreases as $x_{\perp}$ increases.

Before exploring the $T$-dependence of the longitudinal profiles we were interested in the comparison of the electric and magnetic field components. The comparison is clearly visible in Fig. 6.5 for one longitudinal profile. It turns out that at any $q \bar{q}$ separation the parallel electric component is much larger than the other three components which are equal approximately,

$$
1 / 2 E_{\perp}^{2} \approx-1 / 2 B_{\|}^{2} \approx-1 / 2 B_{\perp}^{2}
$$

This holds for both below and above $T_{c}$. The statement also will be true for any other longitudinal as well as the transverse profiles and for lattices of any size as we have checked it on other two lattices.

The parallel electric field strength which is directed along the axis connecting the $q \bar{q}$ pair being largest one states us that there is a force which tries to bind the $q$ and $\bar{q}$ together and this force is larger than the other forces in other directions. 


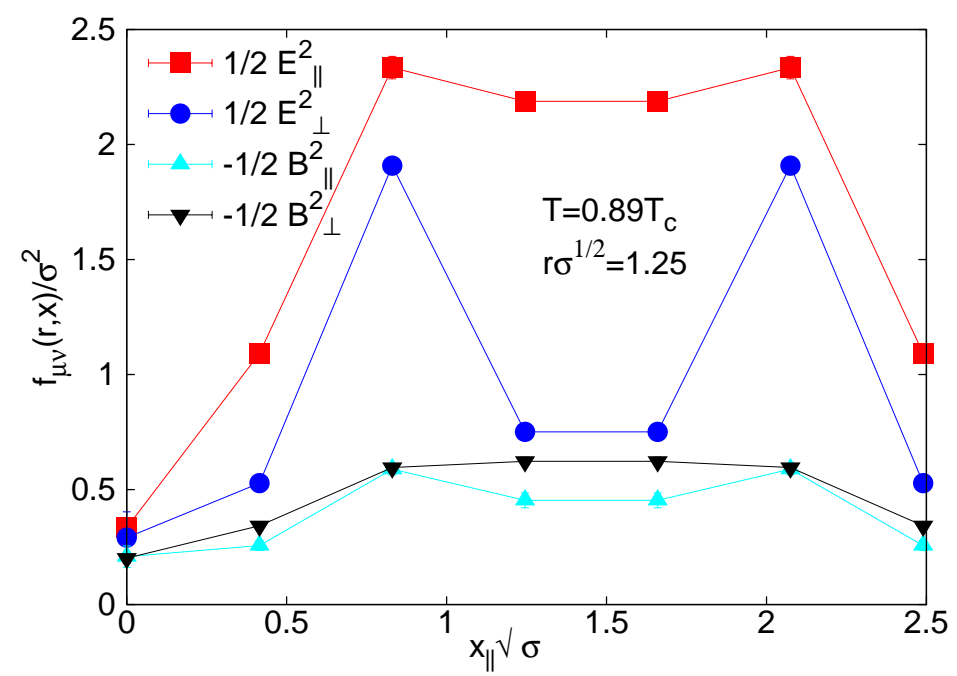

Figure 6.5.: Comparison of $1 / 2 E_{\|}^{2}\left(x_{\perp}\right), 1 / 2 E_{\perp}^{2}\left(x_{\perp}\right),-1 / 2 B_{\|}^{2}\left(x_{\perp}\right)$ and $-1 / 2 B_{\perp}^{2}\left(x_{\perp}\right)$ at $x_{\|}=r / 2$ from $8^{3} \times 4$ lattice.

\subsubsection{Results on the $16 \times 8^{2} \times 4$ lattice}

The results of the longitudinal profiles of the field components on the lattice $16 \times 8^{2} \times 4$ are shown in Figs. 6.6, 6.7, 6.8 and 6.9 at $q \bar{q}$ separations $r \sqrt{\sigma}=1.4,1.7,2.1$ and 2.4 .

On the longitudinal profiles one quark source is placed at $x_{\|}=0$ and another one is at the distance $r$. For the shape of the longitudinal profiles, it has the peak values at the positions of the two Polyakov loops, then it rapidly falls down as the plaquette position goes away from the sources to two opposite directions with $x_{\|}<0$ and $x_{\|}>r$ which belong to the outside of the flux tube. At approximately $x_{\|} \sqrt{\sigma}=-1$ and $r \sqrt{\sigma}+1$ the field strength is almost zero indicating there is no field strength outside the flux tube. The two positions at which the field strength vanishes seem to be the same for all field components as well as for both lattices with $N_{\tau}=4$ and 6 .

In each of the figures there are two data sets differing in temperature in each of the four diagrams that have been plotted at four different values of separation. In these figures, the data corresponding to a higher temperature is expressed by the red square points, while the blue dots are the data at the lower temperature.

It should, first, be explained why there are only two temperature values plotted at a fixed value of separation. In our study the parameters are the $q \bar{q}$ separation $r$, the 

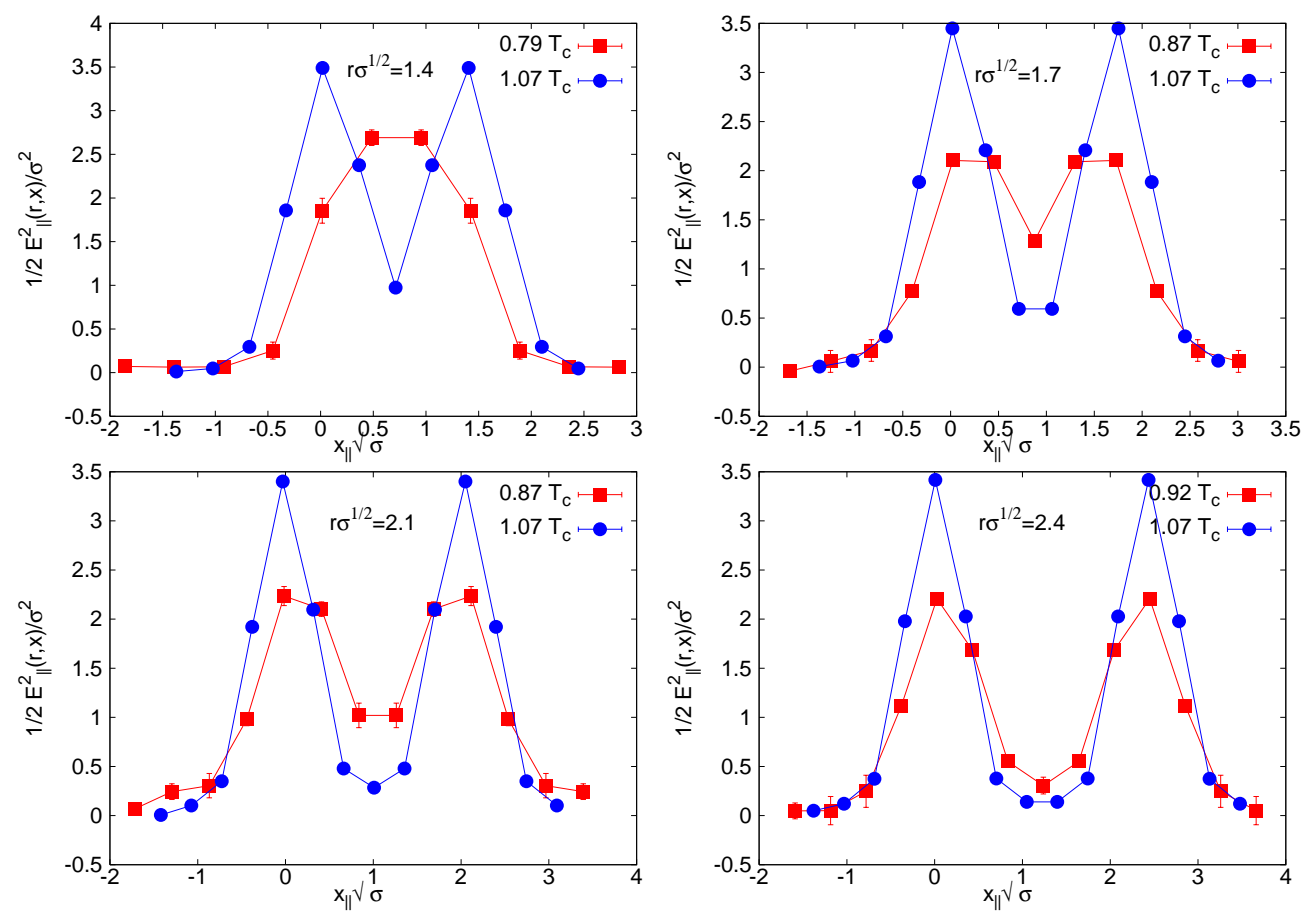

Figure 6.6.: Longitudinal profile $1 / 2 E_{\|}^{2}\left(x_{\|}\right)$at $x_{\perp}=0$ and $r \sqrt{\sigma}=1.4,1.7,2.1$ and 2.4, in units of the string tension, for several values of $T$ from $16 \times 8^{2} \times 4$ lattice.
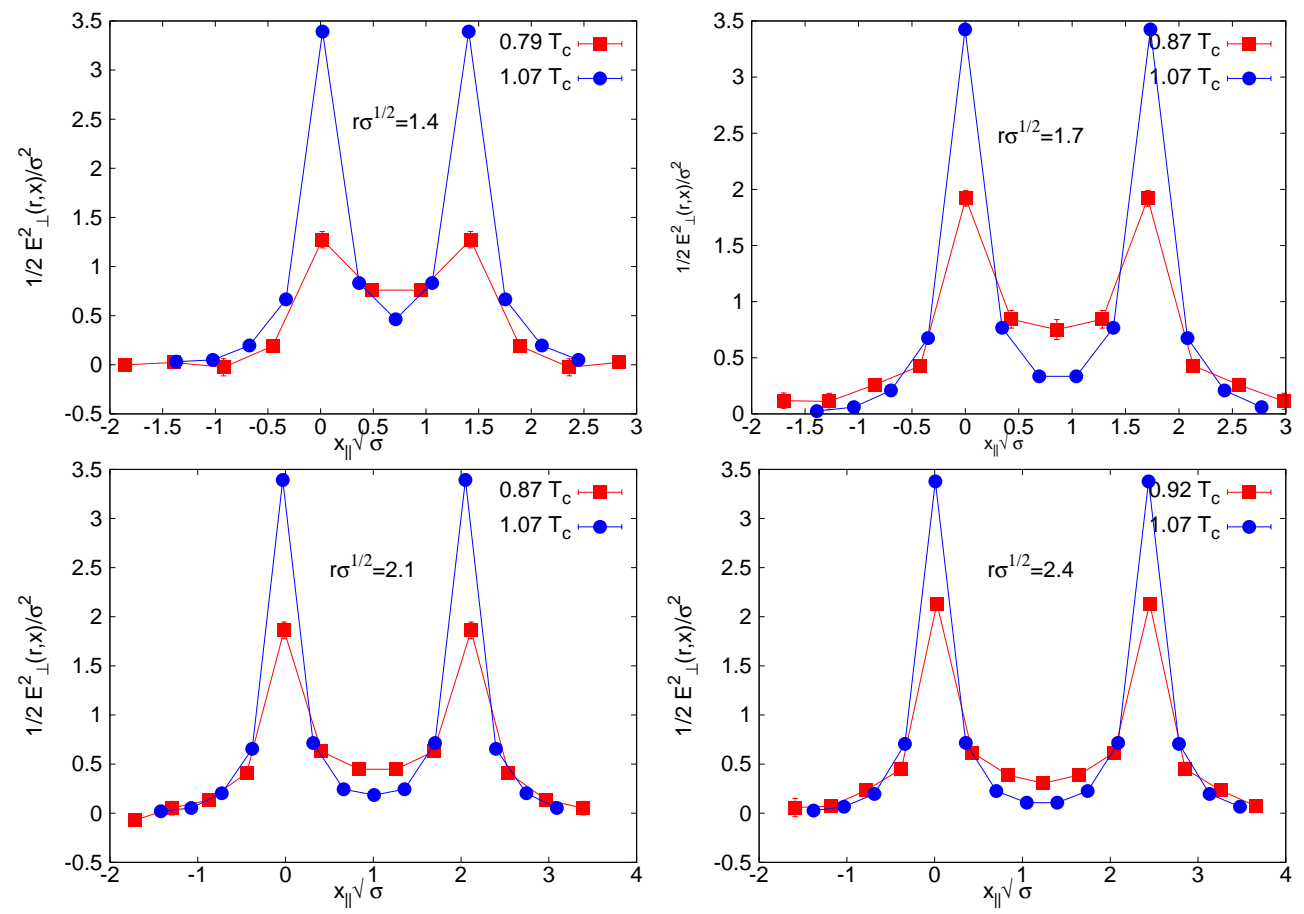

Figure 6.7.: Longitudinal profile $1 / 2 E_{\perp}^{2}\left(x_{\|}\right)$at $x_{\perp}=0$ and $r \sqrt{\sigma}=1.4,1.7,2.1$ and 2.4, in units of the string tension, for several values of $T$ from $16 \times 8^{2} \times 4$ lattice. 

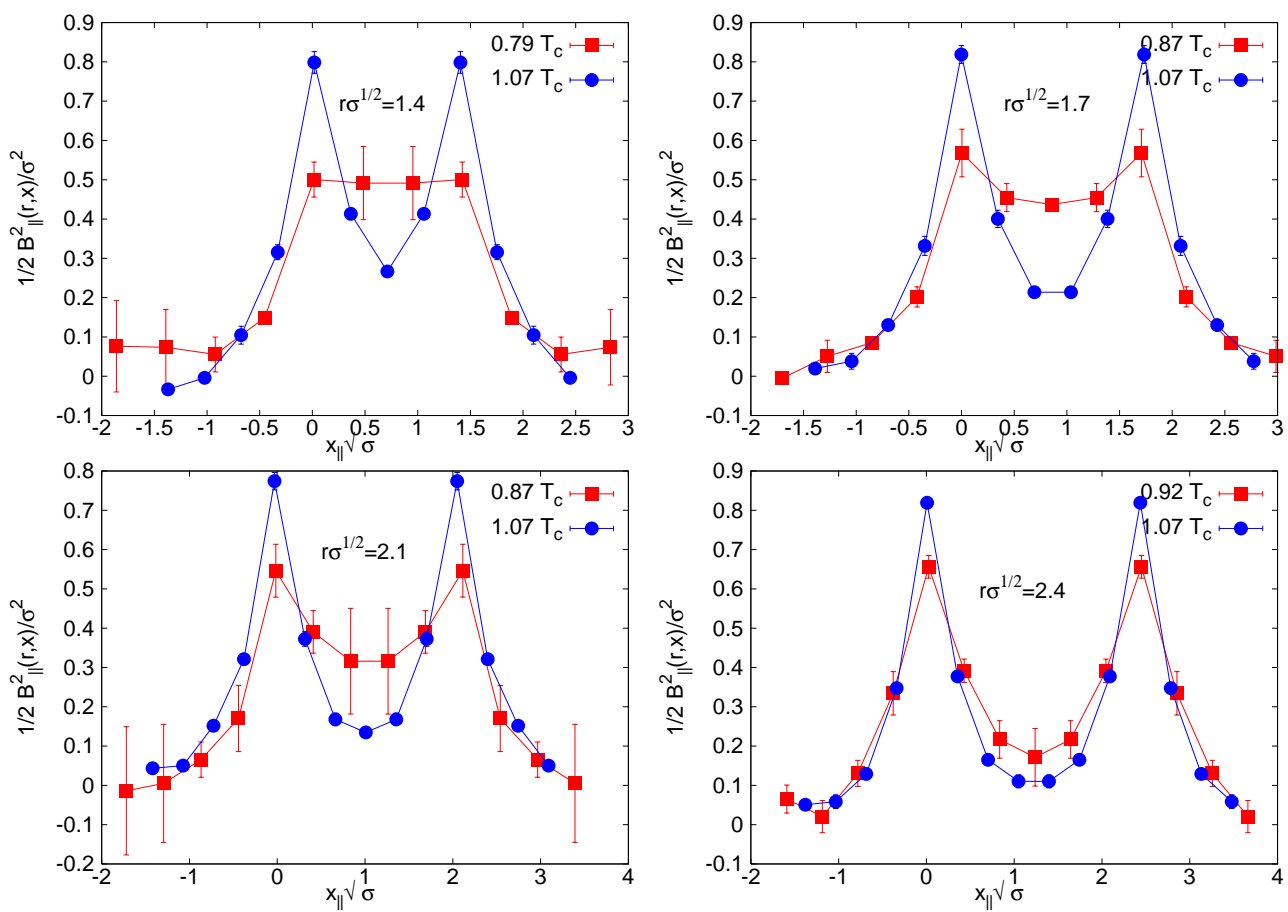

Figure 6.8.: Longitudinal profile $-1 / 2 B_{\|}^{2}\left(x_{\|}\right)$at $x_{\perp}=0$ and $r \sqrt{\sigma}=1.4,1.7,2.1$ and 2.4, in units of the string tension, for several values of $T$ from $16 \times 8^{2} \times 4$ lattice.

plaquette distance $x$ and the coupling constant $\beta$. One must compare results at a fixed value of these parameters. If we want to compare our data in physical units and we transform our parameter values to physical units the $q \bar{q}$ separations that were equal, $r$, in lattice units will be no longer equal because it will be expressed as $r a$ and $a$ takes a different value for each $\beta$. This reduces the number of data to be compared at a fixed value of the $q \bar{q}$ separation. Producing as many as possible lattice data at as many as possible values of $r$ and $\beta$ increases the number of the matching physical separations as it gives many combinations of $r$ and $a$ to the expression $r a$. So the larger the lattice, the more number of data to be plotted as a function of temperature as we reach higher number of values of the $q \bar{q}$ separation. $q \bar{q}$ separation values at $N_{\tau}=4$, in units of the string tension, are summarized in Table. 6.1.

Now let us pay attention to how the temperature changes the longitudinal profiles. Look at the middle region between the two Polyakov loops where the separation takes the largest value. Then it will be clearly seen that the field strength on the middle point between the $q \bar{q}$ pair is going down when the temperature is changed from the lower to the higher. Increase of the peak values, the other way around, indicates a non-scaling of 

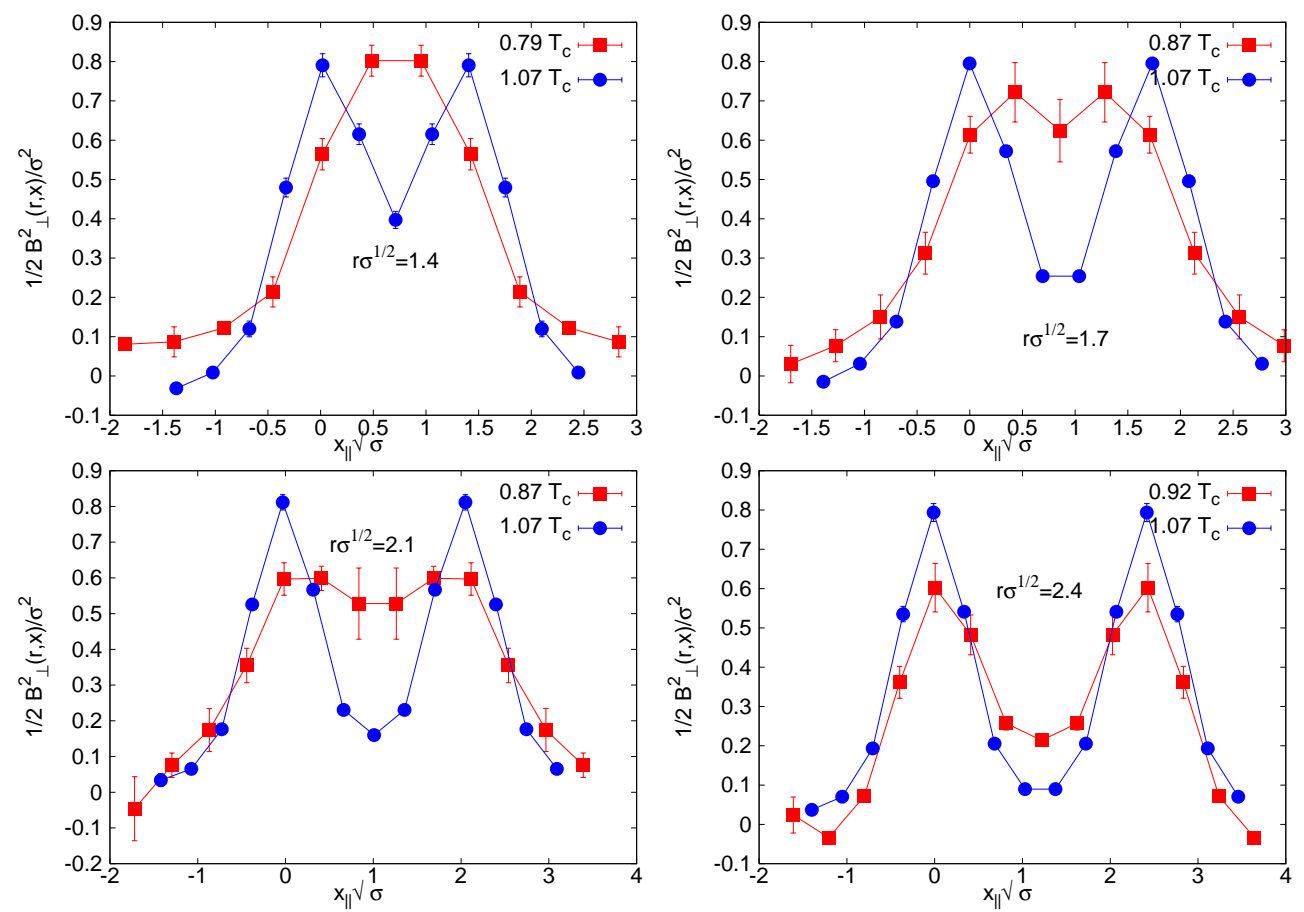

Figure 6.9.: Longitudinal profile of $-1 / 2 B_{\perp}^{2}\left(x_{\|}\right)$at $x_{\perp}=0$ and $r \sqrt{\sigma}=1.4,1.7,2.1$ and 2.4 , in units of the string tension, for several values of $T$ from $16 \times 8^{2} \times 4$ lattice.

\begin{tabular}{|c||c|c|c|c|c|c|c|c|c|}
\hline \multicolumn{10}{|c|}{$N_{\tau}=4$} \\
\hline$\beta$ & $r / a=1$ & $r / a=2$ & $r / a=3$ & $r / a=4$ & $r / a=5$ & $r / a=6$ & $r / a=7$ & $r / a=8$ & $\beta /(a \sqrt{\sigma})^{4}$ \\
\hline \hline 2.20 & 0.469 & 0.938 & 1.407 & 1.876 & 2.345 & 2.814 & 3.283 & 3.752 & 46 \\
2.22 & 0.455 & 0.910 & 1.365 & 1.820 & & & & & 52 \\
2.24 & 0.437 & 0.874 & 1.311 & 1.748 & & & & & 62 \\
2.25 & 0.426 & 0.852 & 1.278 & 1.704 & 2.130 & 2.556 & 2.982 & 3.408 & 68 \\
2.26 & 0.415 & 0.830 & 1.245 & 1.660 & & & & & 76 \\
2.27 & 0.404 & 0.808 & 1.212 & 1.616 & 2.020 & 2.424 & 2.828 & 3.232 & 85 \\
2.28 & 0.393 & 0.786 & 1.179 & 1.572 & & & & & 96 \\
2.29 & 0.381 & 0.762 & 1.143 & 1.524 & 1.905 & 2.286 & 2.667 & 3.048 & 108 \\
2.30 & 0.370 & 0.740 & 1.110 & 1.480 & 1.850 & 2.220 & 2.590 & 2.960 & 123 \\
2.32 & 0.347 & 0.694 & 1.041 & 1.388 & 1.735 & 2.082 & 2.429 & 2.776 & 160 \\
2.40 & 0.265 & 0.530 & 0.795 & 1.060 & & & & & 485 \\
\hline
\end{tabular}

Table 6.1.: $q \bar{q}$ separations, in units of the string tension, at $N_{\tau}=4$. 

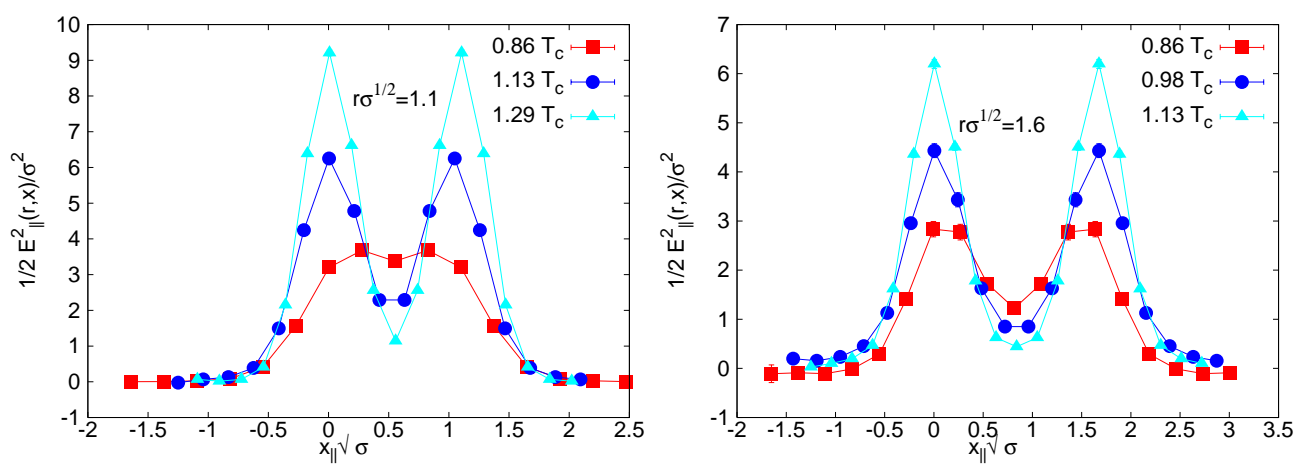

Figure 6.10.: Longitudinal profile $1 / 2 E_{\|}^{2}\left(x_{\|}\right)$at $x_{\perp}=0$ and $r \sqrt{\sigma}=1.1$ and 1.6, in units of the string tension, for several values of $T$ from $24 \times 12^{2} \times 6$ lattice.

the results near to the self energy region. At small distances the flux tube is corrupted by lattice artefacts, which can be understood in terms of lattice perturbation theory [3]. We refrained from solving the problem in the self energy region since we are interested in the physical region which can be considered as the region far from the sources.

This $T$-dependence holds for all electric and magnetic field components. The $T$-dependence of the field strength value on the middle point will be discussed in Sec. 6.7 in more detail.

What one also can see from the figures is the $r$-dependence of the field strength value. To do so one has to compare those data points which are at the same value of the temperature, say $1.07 T_{c}$ in this case, but at different separation. When the separation value goes from $r \sqrt{\sigma}=1.4$ to 2.4 a field strength value is apparently decreasing.

\subsubsection{Results on the $24 \times 12^{2} \times 6$ lattice}

One needs an increase in resolution of the underlying lattice in order to be as close as possible to the continuum limit. The next lattice we tried the measurement was $24 \times 12^{2} \times 6$. The lattice has a finer lattice spacing than it was at $N_{\tau}=4$. In Figs. $6.10,6.11,6.12$ and 6.13 the electric and magnetic field strengths from this lattice are depicted.

As it has already been mentioned in the previous section the larger the lattice, the more data can be plotted as a function of temperature as we obtain more values of the $q \bar{q}$ separation. So we have now three data sets with the same separation. $q \bar{q}$ separation 

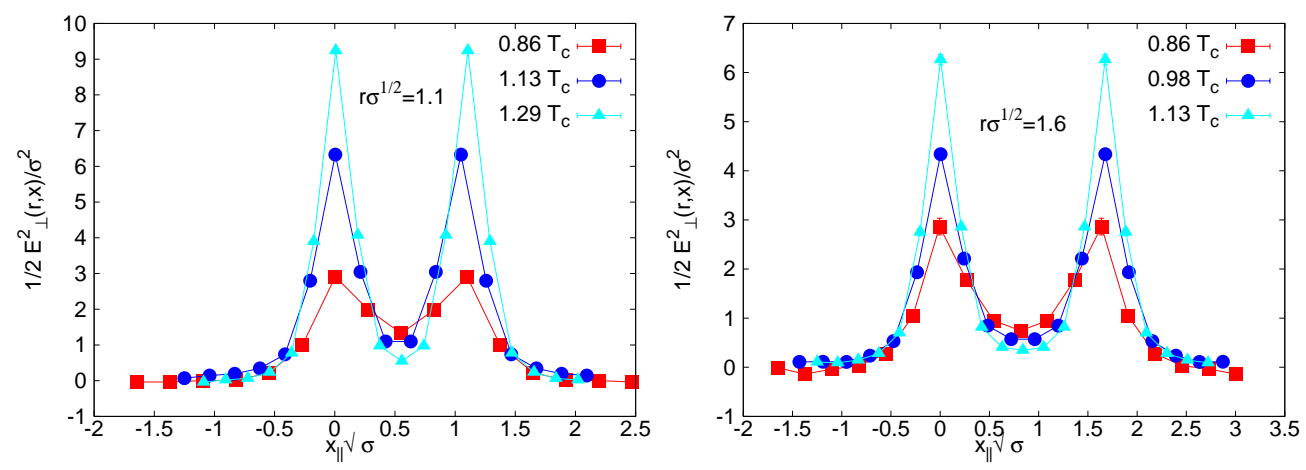

Figure 6.11.: Longitudinal profile $1 / 2 E_{\perp}^{2}\left(x_{\|}\right)$at $x_{\perp}=0$ and $r \sqrt{\sigma}=1.1$ and 1.6, in units of the string tension, for several values of $T$ from $24 \times 12^{2} \times 6$ lattice.
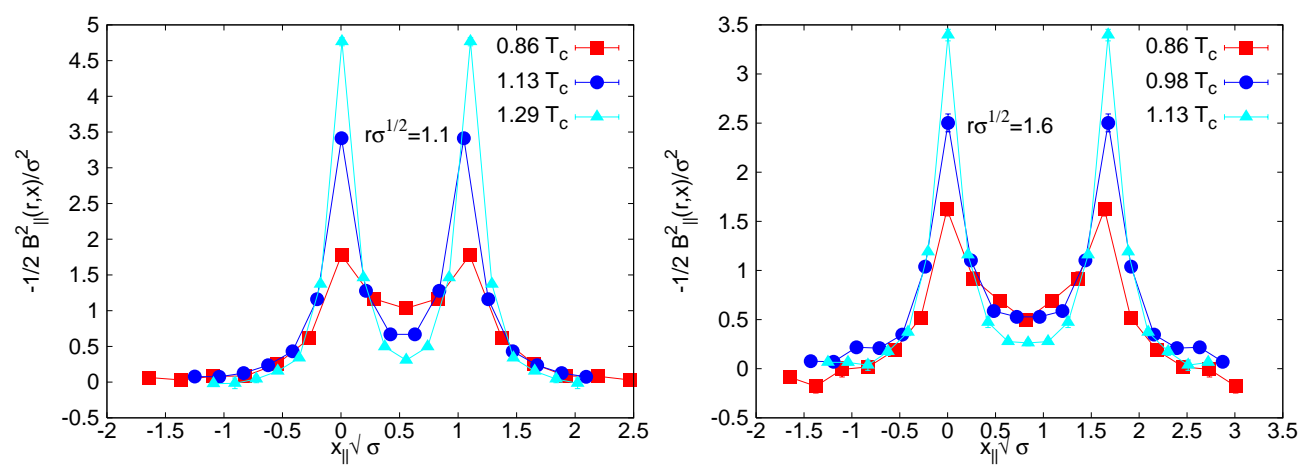

Figure 6.12.: Longitudinal profile $-1 / 2 B_{\|}^{2}\left(x_{\|}\right)$at $x_{\perp}=0$ and $r \sqrt{\sigma}=1.1$ and 1.6, in units of the string tension, for several values of $T$ from $24 \times 12^{2} \times 6$ lattice.
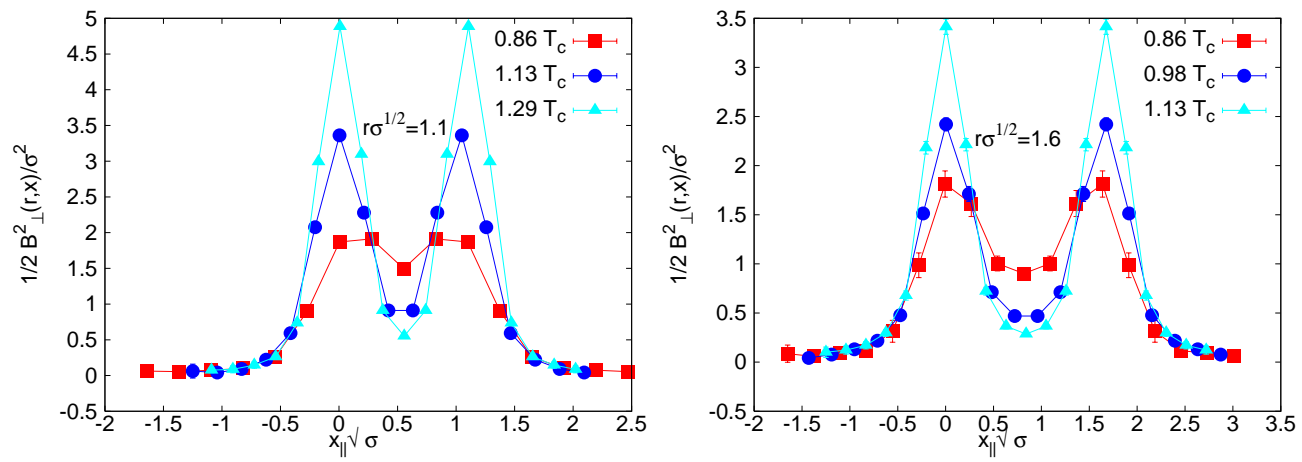

Figure 6.13.: Longitudinal profile $-1 / 2 B_{\perp}^{2}\left(x_{\|}\right)$at $x_{\perp}=0$ and $r \sqrt{\sigma}=1.1$ and 1.6, in units of the string tension, for several values of $T$ from $24 \times 12^{2} \times 6$ lattice. 


\begin{tabular}{|c||c|c|c|c|c|c|c|c|c|}
\hline \multicolumn{10}{|c|}{$N_{\tau}=6$} \\
\hline$\beta$ & $r / a=1$ & $r / a=2$ & $r / a=3$ & $r / a=4$ & $r / a=5$ & $r / a=6$ & $r / a=7$ & $r / a=8$ & $\beta /(a \sqrt{\sigma})^{4}$ \\
\hline \hline 2.31 & 0.358 & 0.716 & 1.074 & 1.432 & 1.790 & 2.148 & 2.506 & 2.864 & 140 \\
2.35 & 0.314 & 0.628 & 0.942 & 1.256 & 1.570 & 1.884 & 2.198 & 2.512 & 241 \\
2.39 & 0.274 & 0.548 & 0.822 & 1.096 & 1.370 & 1.644 & 1.918 & 2.192 & 421 \\
2.43 & 0.239 & 0.478 & 0.717 & 0.956 & 1.195 & 1.434 & 1.673 & 1.912 & 739 \\
2.47 & 0.209 & 0.418 & 0.627 & 0.836 & 1.045 & 1.254 & 1.463 & 1.672 & 1293 \\
2.51 & 0.183 & 0.366 & 0.549 & 0.732 & 0.915 & 1.098 & 1.281 & 1.464 & 2240 \\
\hline
\end{tabular}

Table 6.2.: $q \bar{q}$ separations, in units of the string tension, at $N_{\tau}=6$.
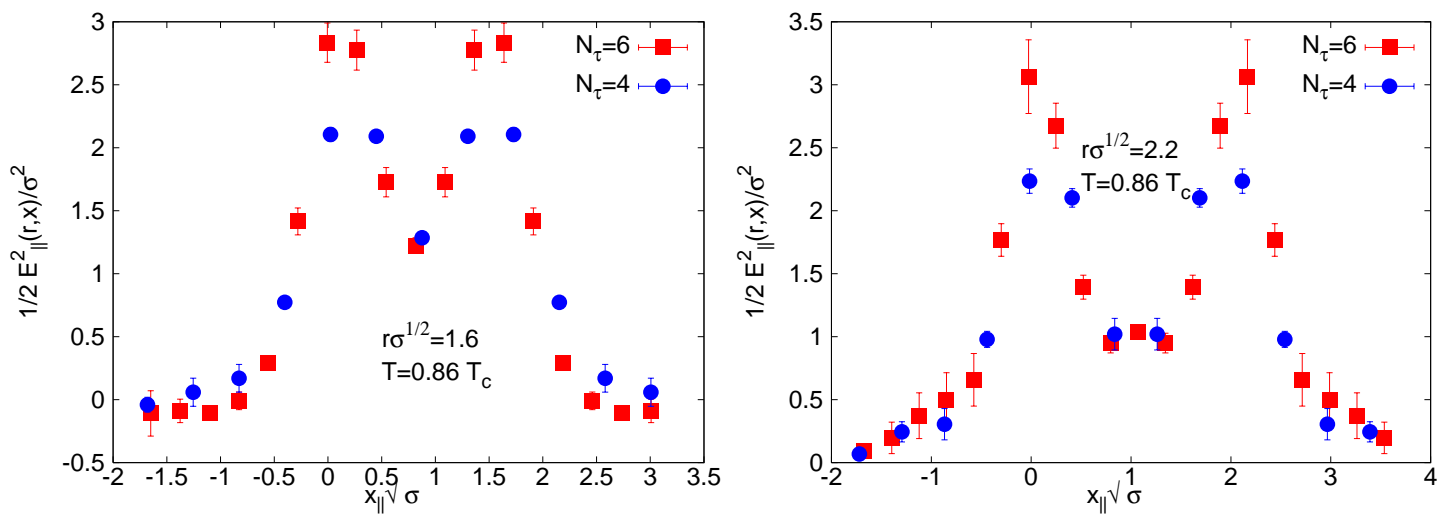

Figure 6.14.: Comparison of $1 / 2 E_{\|}^{2}\left(x_{\|}\right)$at $x_{\perp}=0$ for $T=0.86 T_{c}$ from two lattices differing in lattice spacing $a$. Left: $r \sqrt{\sigma}=1.6 ;$ Right: $r \sqrt{\sigma}=2.2$.

values at $N_{\tau}=6$, in units of the string tension, are summarized in Table. 6.2.

On this lattice the two separation values $r \sqrt{\sigma}=1.1$ and 1.6 were chosen to show the $T$-dependence of the field strength. The same $T$ as well as $r$ dependence as they were for $N_{\tau}=4$ are seen for all field strengths on the middle point between the $q \bar{q}$ pair. But since the lattice has a finer lattice spacing than it was at $N_{\tau}=4$ there should be differences in the field strength values. The effect that is caused by a decrease of the lattice spacing is called finite a effects which is going to be discussed in the next section.

\subsubsection{Finite $a$ Effects}

The finite $a$ effects on our results can be seen from the plots given in Fig. 6.14. Here we have chosen $1 / 2 E_{\|}^{2}\left(x_{\|}\right)$at $x_{\perp}=0$ for $T=0.86 T_{c}$ to be compared from two different lattices as an example. 
The data denoted by red square points have been obtained from the lattice with $N_{\tau}=6$ which has a finer lattice spacing. Though the data away from the Polyakov loops locations are compatible to each other within error bars, the values obtained at the finer lattice spacing tend to be systematically smaller than the value corresponding at smaller $N_{\tau}=4$ value. The same is found in a comparison of the transverse profiles. Note, however, that the self energy peaks at the positions of the Polyakov loops become higher.

At the larger $q \bar{q}$ separation which is on the right of the figure one can also notice that the effect becomes smaller than at the smaller $q \bar{q}$ separation. Overall, however, discretization effects appear to be small enough for the present purpose of the investigation about $T$-dependence of the field strengths.

Above we have seen the longitudinal profiles at $y=0$ as well as $z=0$ of the surface plots shown in Section. 6.1. In principle, it is possible to see longitudinal profiles also at $x_{\perp} \neq 0$, where $x_{\perp}=\sqrt{y^{2}+z^{2}}$. These profiles would show decreasing field strengths when $x_{\perp}$ is raised, as will be discussed in the next section.

\subsection{Transverse Profiles}

A transverse profile of a field strength shows how the field strength value is spread along the direction perpendicular to the axis connecting $q \bar{q}$ pair at a fixed constant $x_{\|}$. Throughout the work we looked at the transverse profiles at $x_{\|}=r / 2$ as the middle plane between the $q$ and $\bar{q}$ can be considered as the physical region where the self energy contributions of the sources are negligible and also equally far from the sources.

With the finite $a$ effects discussed in the previous section, here we only show the results from the lattice with $N_{\tau}=6$. The number of lattice sites in the transverse direction investigated is $N_{\perp} / 4+1=4$ for the lattice of size $24 \times 12^{2} \times 6$. The transverse distance is $x_{\perp}=\sqrt{y^{2}+z^{2}}$ and it contains not only on-axis distances, $y \neq 0, z=0$ or $y=0$, $z \neq 0$, but also off-axis distances corresponding to $y \neq 0, z \neq 0$.

Transverse profiles of the field components on the middle point $x_{\|}=r / 2$ from the lattice of size $24 \times 12^{2} \times 6$ are shown in Figs. 6.15, 6.16, 6.17 and 6.18. 

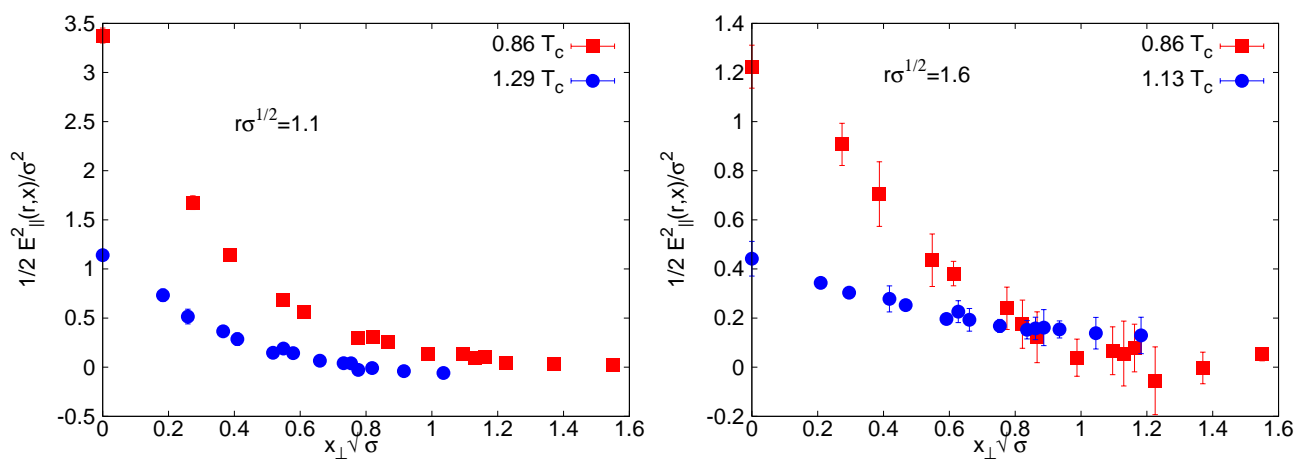

Figure 6.15.: Transverse profile $1 / 2 E_{\|}^{2}\left(x_{\perp}\right)$ on the middle point $x_{\|}=r / 2$ at $r \sqrt{\sigma}=1.1$ and 1.6, in units of the string tension, for several values of $T$ from $24 \times 12^{2} \times 6$ lattice.
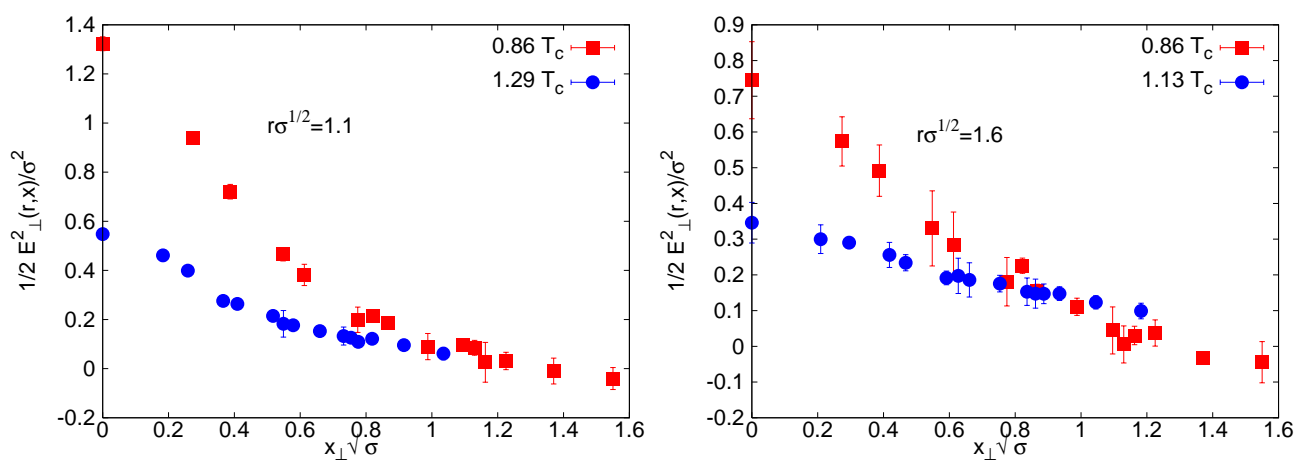

Figure 6.16.: Transverse profile $1 / 2 E_{\perp}^{2}\left(x_{\perp}\right)$ on the middle point $x_{\|}=r / 2$ at $r \sqrt{\sigma}=1.1$ and 1.6, in units of the string tension, for several values of $T$ from $24 \times 12^{2} \times 6$ lattice.
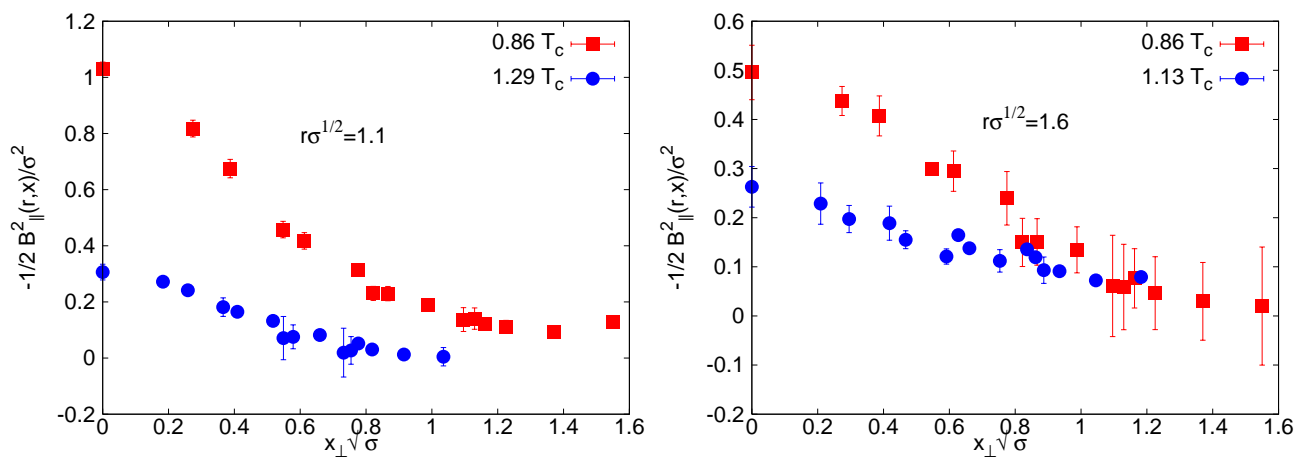

Figure 6.17.: Transverse profile $-1 / 2 B_{\|}^{2}\left(x_{\perp}\right)$ on the middle point $x_{\|}=r / 2$ at $r \sqrt{\sigma}=1.1$ and 1.6, in units of the string tension, for several values of $T$ from $24 \times 12^{2} \times 6$ lattice. 

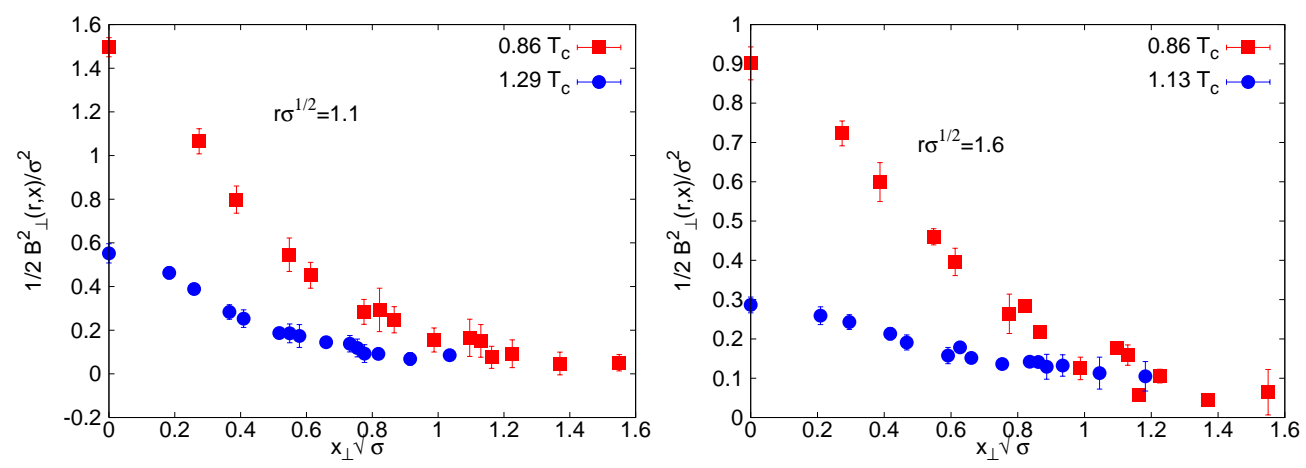

Figure 6.18.: Transverse profile $-1 / 2 B_{\perp}^{2}\left(x_{\perp}\right)$ on the middle point $x_{\|}=r / 2$ at $r \sqrt{\sigma}=1.1$ and 1.6, in units of the string tension, for several values of $T$ from $24 \times 12^{2} \times 6$ lattice.

The transverse profiles are those profiles the starting values at $x_{\perp}=0$ of which are exactly the midpoints between the two Polyakov loops on the longitudinal profiles shown in Figs. 6.10, 6.11, 6.12 and 6.13 at each separation value. The starting maximum value, then, decreases as it goes away from the axis connecting the $q \bar{q}$ pair.

From what we see on the plots, we are led to the conclusion that there are three factors that lead field strength values to decrease when those factors increase: the transverse distance $x_{\perp}, q \bar{q}$ separation $r$ and temperature $T$.

- when a plaquette goes away from the axis connecting the $q \bar{q}$ pair along the perpendicular direction the field strength value rapidly falls down and goes to zero;

- if we separate $q$ and $\bar{q}$ sources further away from each other the field strength decreases compared to its previous value at the smaller $q \bar{q}$ separation;

- the decrease with increasing temperature in which we are interested in this work.

In the shown figures the two temperature values are both from below and above $T_{c}$ presenting how a transverse profile is changing when it undergoes the deconfining phase transition. Not only the maximum field strength value at $x_{\perp} \sqrt{\sigma}=0$ but also the field strengths at all $x_{\perp} \sqrt{\sigma}>0$ are falling down with rising temperature.

What else one can see at first sight from the transverse profiles is a $T$-dependence of the half width, $D$, of the flux tube. It is already visible that the half width of the transverse profile is decreasing with rising temperature. Denote an initial field strength at a lower temperature $T_{1}$ by $f_{1}$ and a final one at a higher temperature $T_{2}$ by $f_{2}$. The maximum 


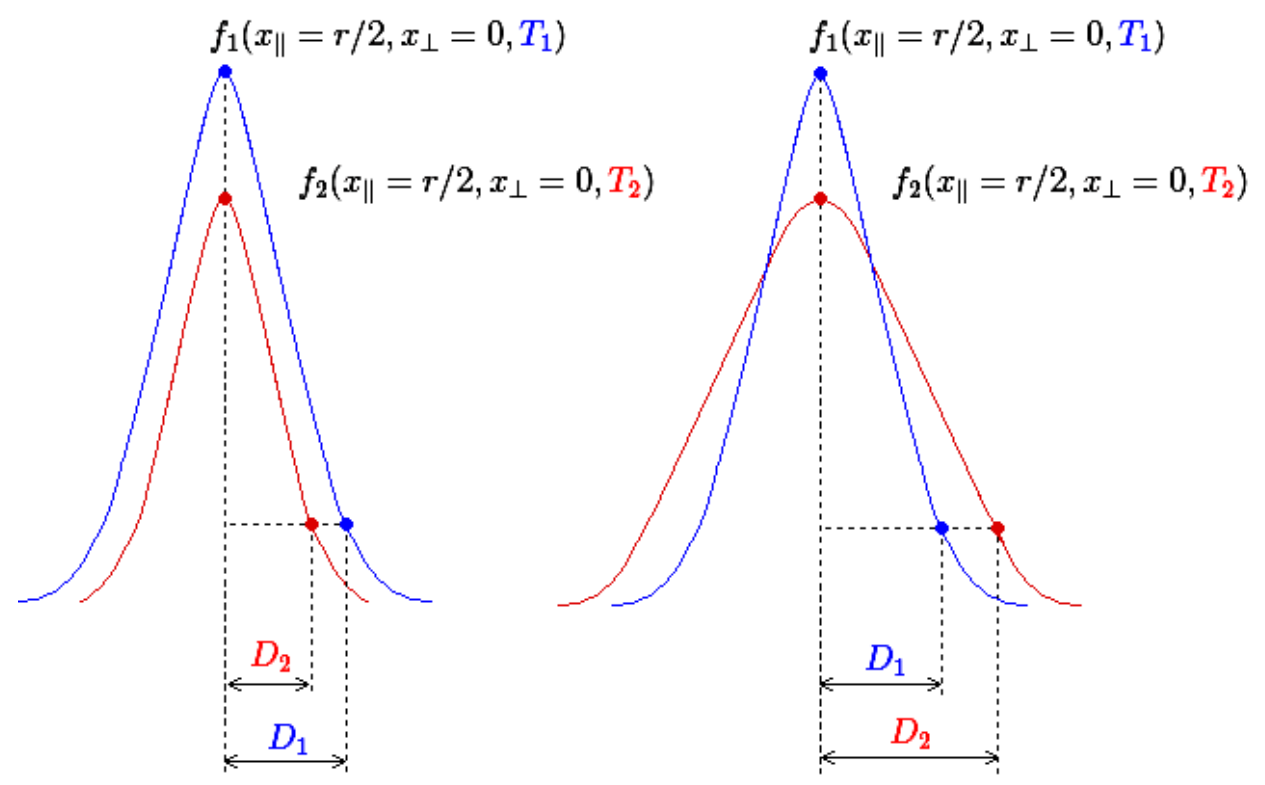

Figure 6.19.: Two possible szenarios of the change of transverse profiles with temperature.

field strength value at $x_{\perp}=0$ decreases with rising temperature as we have seen at the middle point of the longitudinal profiles. So $f_{1}\left(x_{\perp}=0\right)$ decreases to $f_{2}\left(x_{\perp}=0\right)$ for both left and right part of Fig. 6.19 like it has been drawn. In Fig. 6.19 the difference is that the width is decreasing for the left figure while it is increasing for the right figure.

Our main task is to find out whether the transverse profiles look like the left figure or look like the right figure in the sketch illustrated in Fig. 6.19.

If the width decreases with temperature, as in the left of Fig. 6.19, then the equality

$$
f_{1}\left(r, x_{\perp}^{1} \sqrt{\sigma}, T_{1}\right)=f_{2}\left(r, x_{\perp}^{2} \sqrt{\sigma}, T_{2}\right)
$$

is reached at perpendicular distances $x_{\perp}^{1} \sqrt{\sigma}>x_{\perp}^{2} \sqrt{\sigma}$, and vice versa.

It is seen from the transverse profiles that $x_{\perp}^{1} \sqrt{\sigma}>x_{\perp}^{2} \sqrt{\sigma}$ or $D_{1}>D_{2}$ at all $q \bar{q}$ separation values if $T_{1}<T_{2}$ where $x_{\perp}^{1} \sqrt{\sigma}$ and $x_{\perp}^{2} \sqrt{\sigma}$ are the transverse coordinates of the plaquette the temperature of which corresponds to $T_{1}$ and $T_{2}$ respectively.

Because of the symmetry of the distribution relative to the axis connecting the $q \bar{q}$ pair 
we did not plot another half width which whould have been plotted at negative $x_{\perp} \sqrt{\sigma}$.

Quantitative analysis on the width will be done in detail in Sec. 6.5 by means of the fit to the transverse profiles and its results will be discussed in Sec. 6.6.

\subsection{Energy and Action densities}

After we have investigated the profiles of the basic ingredients which compose the energy and action densities of the flux tube we can now start to discuss the energy and action densities. The combinations of total electric and magnetic field components define the total energy $\varepsilon$ and action densities $\delta$ by Eqs. 4.6 and 4.7. From now on all our analysis as well as the fitting procedure for the determination of the width of the flux tube will be done on our largest lattice of size $24 \times 12^{2} \times 6$.

The magnetic contributions are negative, while electric contributions are positive. Thus, there is a cancellation between the two terms in Eq. 4.6, while the action density is amplified in Eq. 4.7. We can thus expect that the action density $\delta$ is much larger than the energy density $\varepsilon$ and is also more broader around the two sources. Indeed, it is clearly seen from the surface plots in Figs. 6.20, 6.21 and 6.22 that the action density value, $\delta\left(x_{\|}, x_{\perp}\right)$, is about $3-4$ times larger than the energy density value, $\varepsilon\left(x_{\|}, x_{\perp}\right)$.

In the left columns of each figure the energy density distribution $\varepsilon\left(x_{\|}, x_{\perp}\right)$ is depicted, while in the right columns the action density distribution $\delta\left(x_{\|}, x_{\perp}\right)$ is depicted. In Fig. 6.20 they have been plotted for the temperature $T=0.86 T_{c}$ which is below $T_{c}$ and for the temperatures $T=1.13 T_{c}$ and $1.29 T_{c}$ which are above $T_{c}$ at $q \bar{q}$ separation $r \sqrt{\sigma}=1.1$, while in Fig. 6.21 the same are plotted but for $T=0.65 T_{c}$ and $0.98 T_{c}$ below $T_{c}$ and for $1.29 T_{c}$ above $T_{c}$ at $r \sqrt{\sigma}=1.4$ and in Fig. 6.22 the situation for $T=0.86 T_{c}$ and $0.98 T_{c}$ below $T_{c}$ and for $1.13 T_{c}$ above $T_{c}$ at $r \sqrt{\sigma}=1.6$ is shown.

The remarks on geometrical symmetry as well as on the general $T$ and $r$ dependence as discussed in the context of the electric and magnetic field strength distributions apply as well for the energy and action densities. Both, energy and action density distributions look smoother than their components' distributions due to the cancellation of some fluctuations when they are computed with the Eqs. (4.6) and (4.7). 

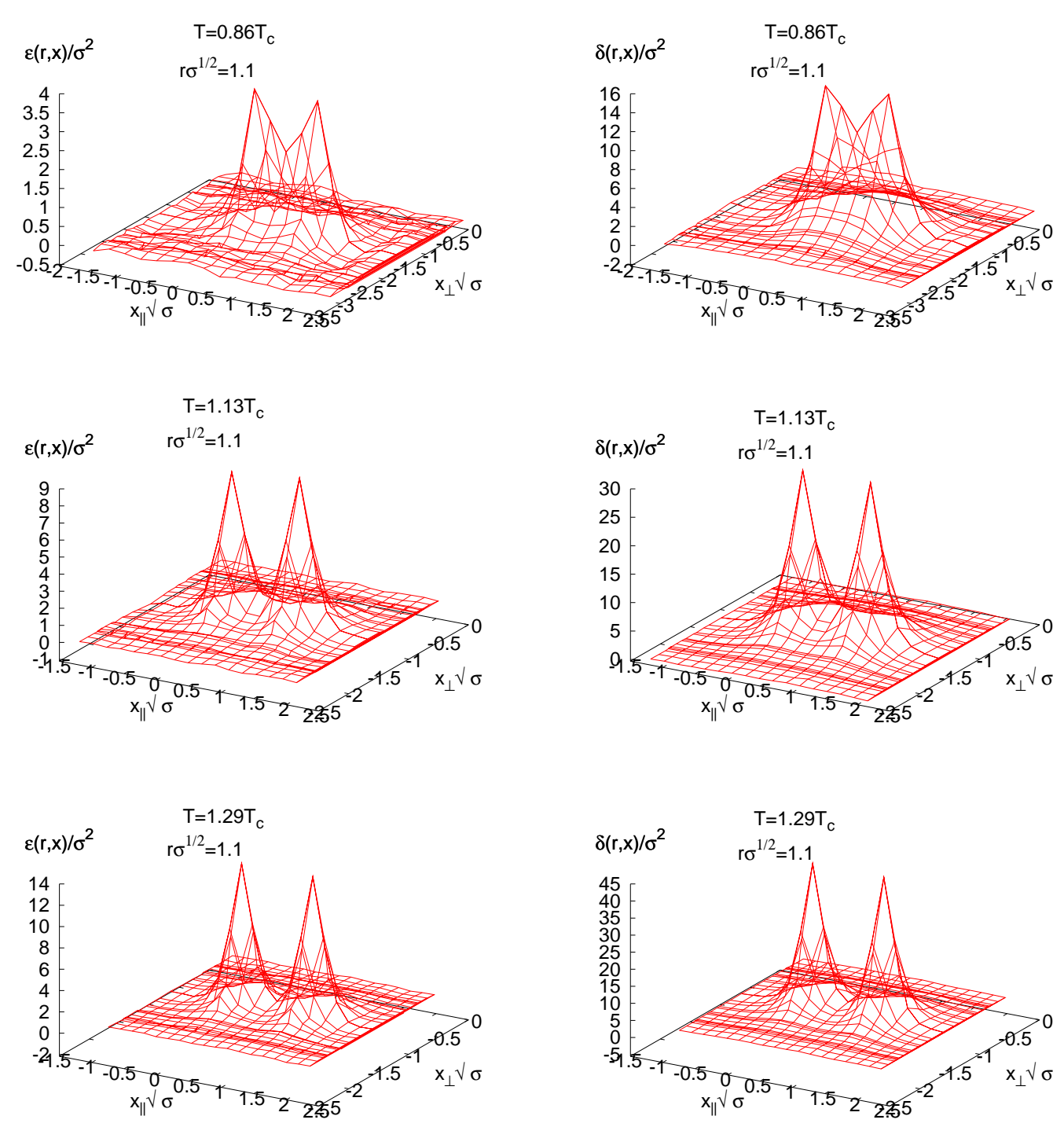

Figure 6.20.: Left: The energy density distribution $\varepsilon\left(x_{\|}, x_{\perp}\right)$ and Right: the action density distribution $\delta\left(x_{\|}, x_{\perp}\right)$ in units of the string tension at $r \sqrt{\sigma}=1.1$ for $T=0.86 T_{c}, 1.13 T_{c}$ and $1.29 T_{c}$ from $24 \times 12^{2} \times 6$ lattice. 

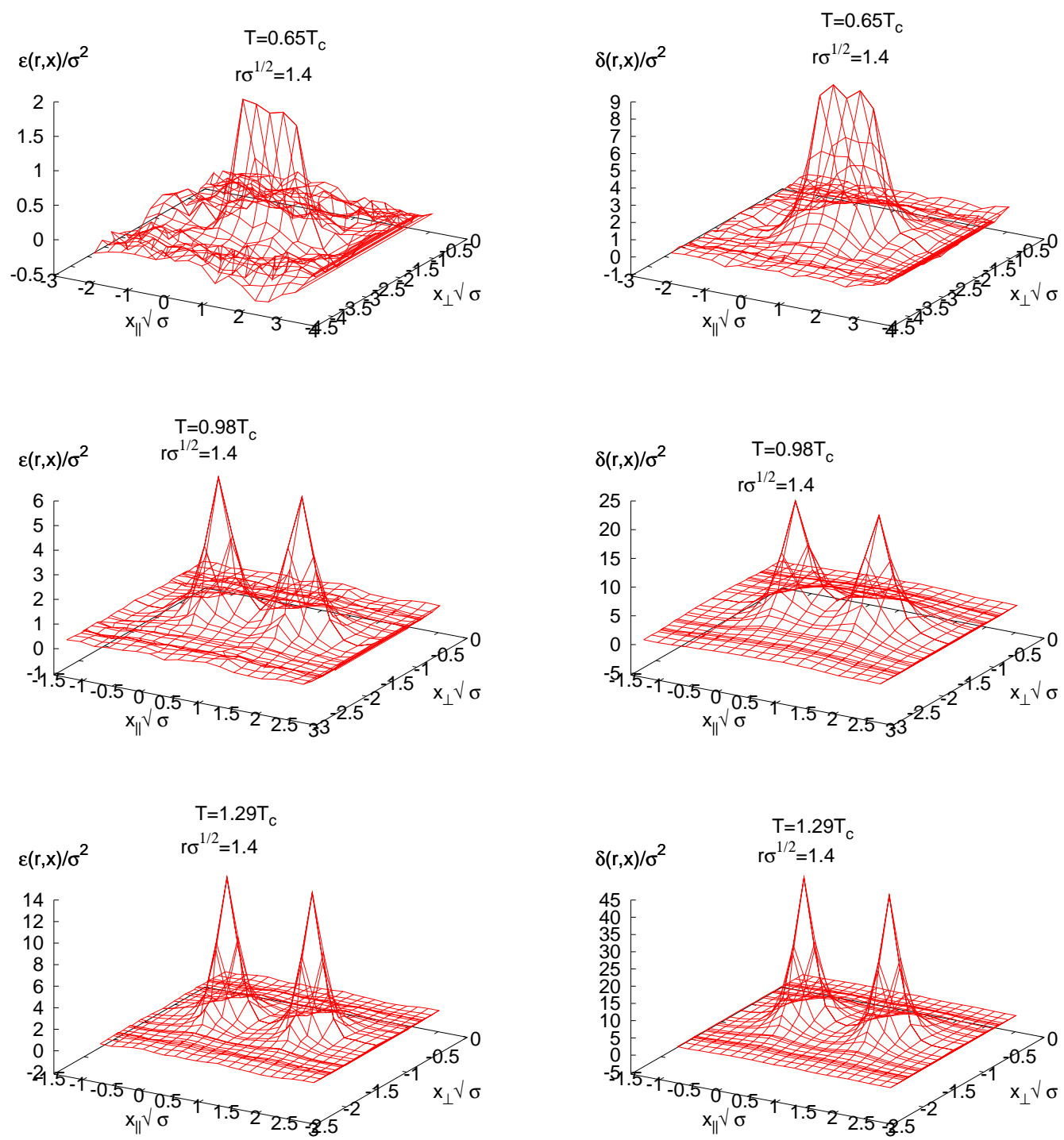

Figure 6.21.: Left: The energy density distribution $\varepsilon\left(x_{\|}, x_{\perp}\right)$ and Right: the action density distribution $\delta\left(x_{\|}, x_{\perp}\right)$ in units of the string tension at $r \sqrt{\sigma}=1.4$ for $T=0.65 T_{c}, 0.98 T_{c}$ and $1.29 T_{c}$ from $24 \times 12^{2} \times 6$ lattice. 

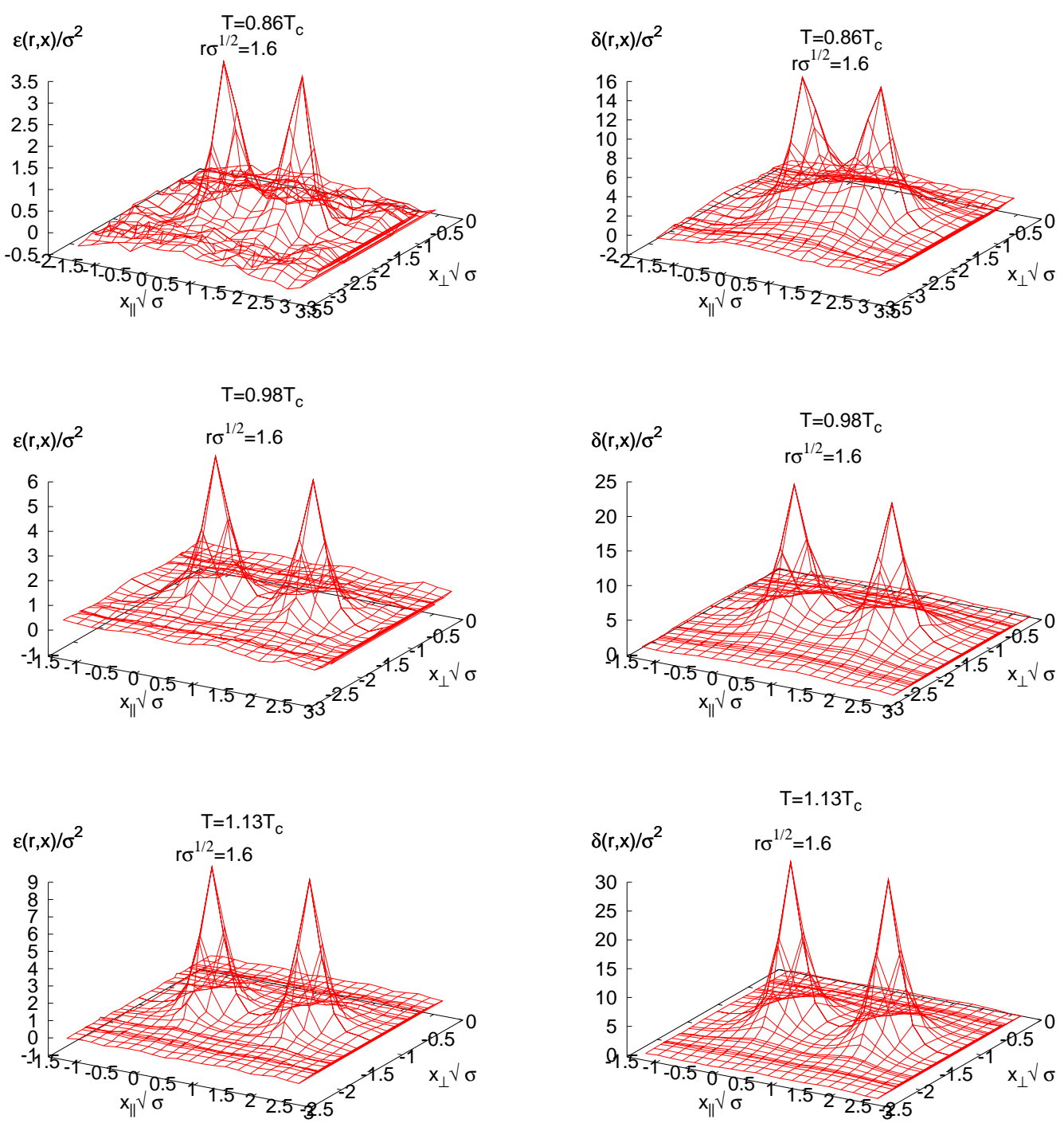

Figure 6.22.: Left: The energy density distribution $\varepsilon\left(x_{\|}, x_{\perp}\right)$ and Right: the action density distribution $\delta\left(x_{\|}, x_{\perp}\right)$ in units of the string tension at $r \sqrt{\sigma}=1.6$ for $T=0.86 T_{c}, 0.98 T_{c}$ and $1.13 T_{c}$ from $24 \times 12^{2} \times 6$ lattice. 

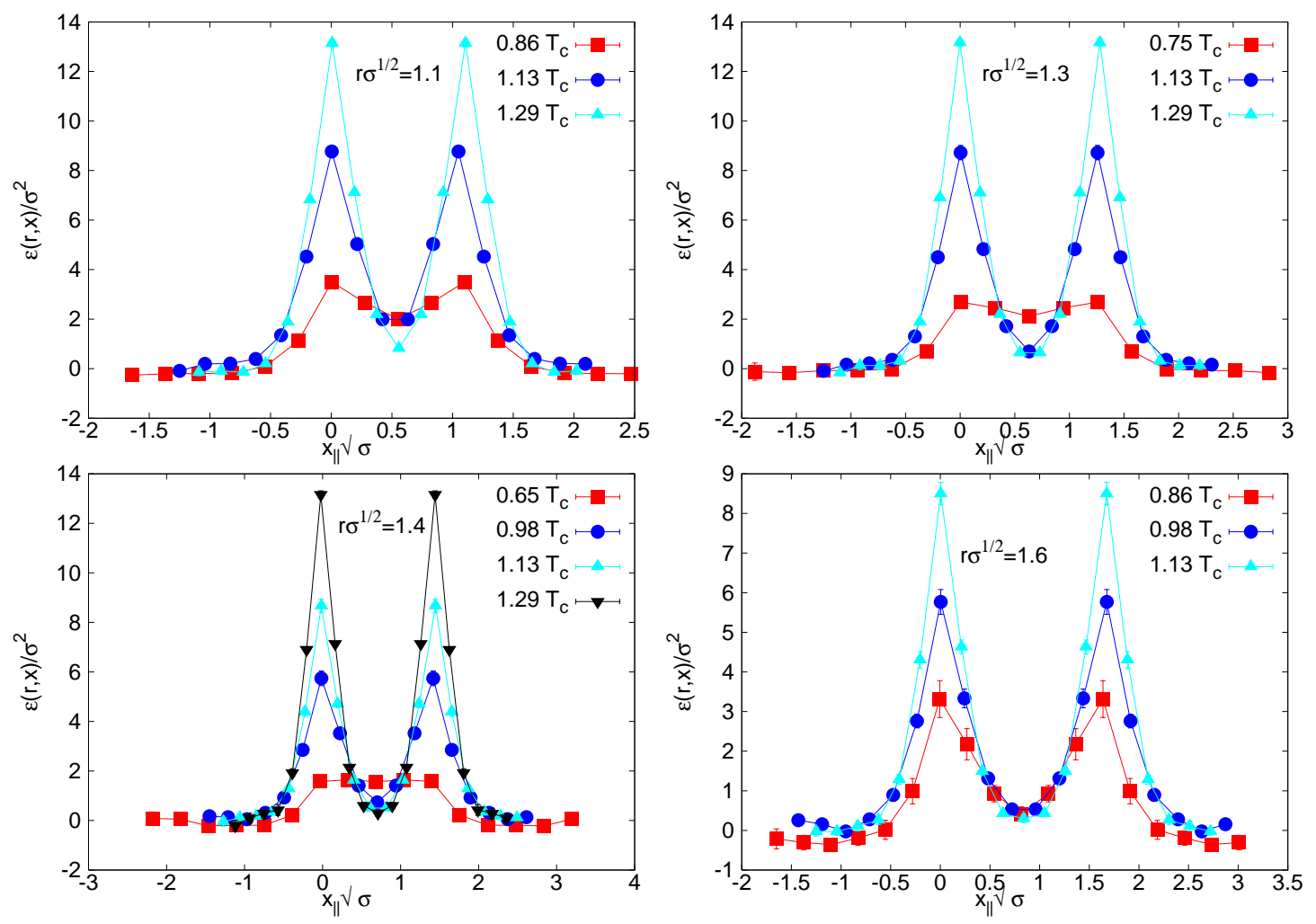

Figure 6.23.: Longitudinal profile $\varepsilon\left(x_{\|}, x_{\perp}=0\right)$ at $r \sqrt{\sigma}=1.1,1.3,1.4$ and 1.6 for several values of temperatures from $24 \times 12^{2} \times 6$ lattice.

If one chooses a column of one of the figures and looks into the temperature increasing direction one will be able to observe the melting or disappearance of the flux tubes. The energy or action density in the region between two sources is eventually disappearing with rising temperature at a fixed $r \sqrt{\sigma}$ value. Above $T_{c}$, in particular at $1.29 T_{c}$ the sources are completely isolated from each other. We will see the numerical indication of this isolation or disappearance in Sec. 6.7.

Let us now look through the longitudinal profiles of the above shown energy and action density distributions. The longitudinal profiles at $x_{\perp}=0$ of the energy $\varepsilon\left(x_{\|}, x_{\perp}\right)$ and action densities $\delta\left(x_{\|}, x_{\perp}\right)$ at $r \sqrt{\sigma}=1.1,1.3,1.4$ and 1.6 for several values of temperatures are shown in Figs. 6.23 and 6.24, where one can more easily watch the $T$-dependence of the density values.

From these profiles one can see that the energy density $\varepsilon$ at $x_{\|}=r / 2, x_{\perp}=0$ and 

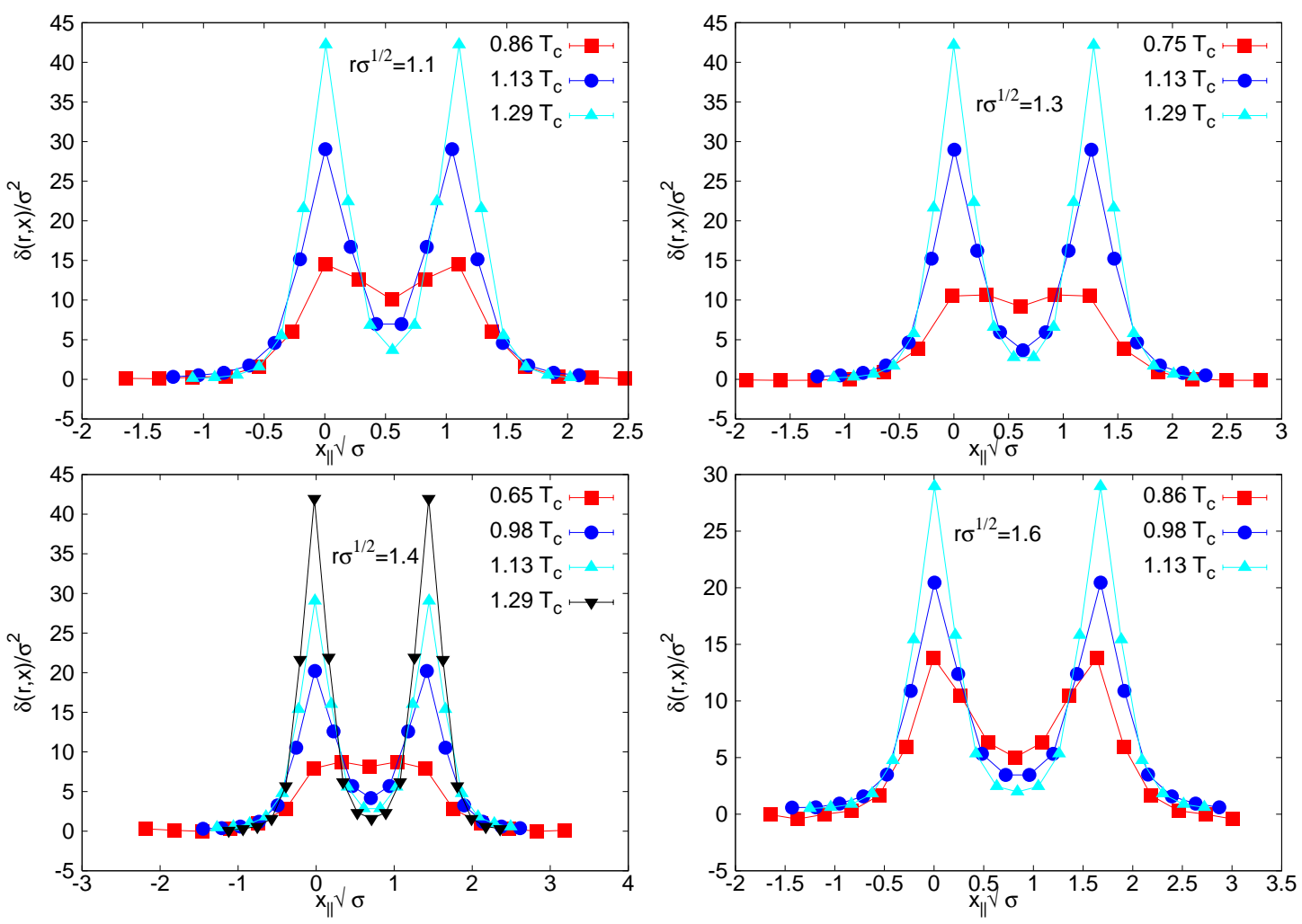

Figure 6.24.: Longitudinal profile $\delta\left(x_{\|}, x_{\perp}=0\right)$ at $r \sqrt{\sigma}=1.1,1.3,1.4$ and 1.6 for several values of temperatures from $24 \times 12^{2} \times 6$ lattice. 

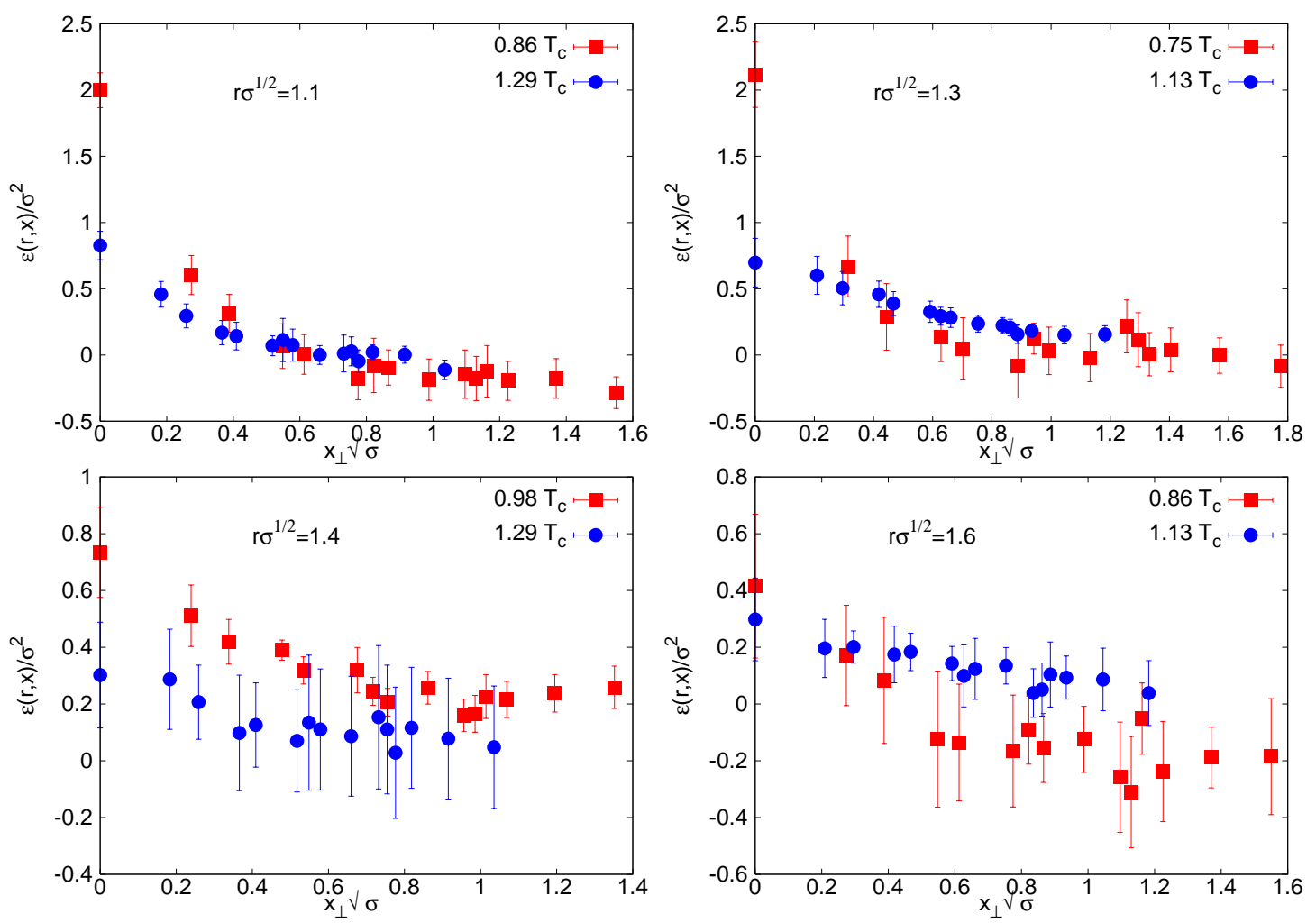

Figure 6.25.: Transverse profile $\varepsilon\left(x_{\|}=r / 2, x_{\perp}\right)$ at $r \sqrt{\sigma}=1.1,1.3,1.4$ and 1.6 for several values of temperatures from $24 \times 12^{2} \times 6$ lattice.

$r \sqrt{\sigma}=1.1$ is decreasing from about 2 to 0.8 when the temperature varies from $0.86 T_{c}$ to $1.29 T_{c}$. For $r \sqrt{\sigma}=1.4$, it is decreasing from about 0.75 to 0.3 when the temperature varies from $0.98 T_{c}$ to $1.29 T_{c}$. The same quantitative estimation can be done for the action density $\delta$.

The transverse profiles at $x_{\|}=r / 2$ of the energy $\varepsilon\left(x_{\|}, x_{\perp}\right)$ and action densities $\delta\left(x_{\|}, x_{\perp}\right)$ at the same values of the $q \bar{q}$ separation and temperature are shown in Figs. 6.25 and 6.26. The transverse profiles are those profiles the starting values at $x_{\perp}=0$ of which are exactly the midpoints between the two Polyakov loops on the longitudinal profiles shown in Figs. 6.23 and 6.24 at each separation value. The starting maximum value, then, decreases as the plaquette position moves away from the axis connecting the $q \bar{q}$ pair in the transverse direction. 

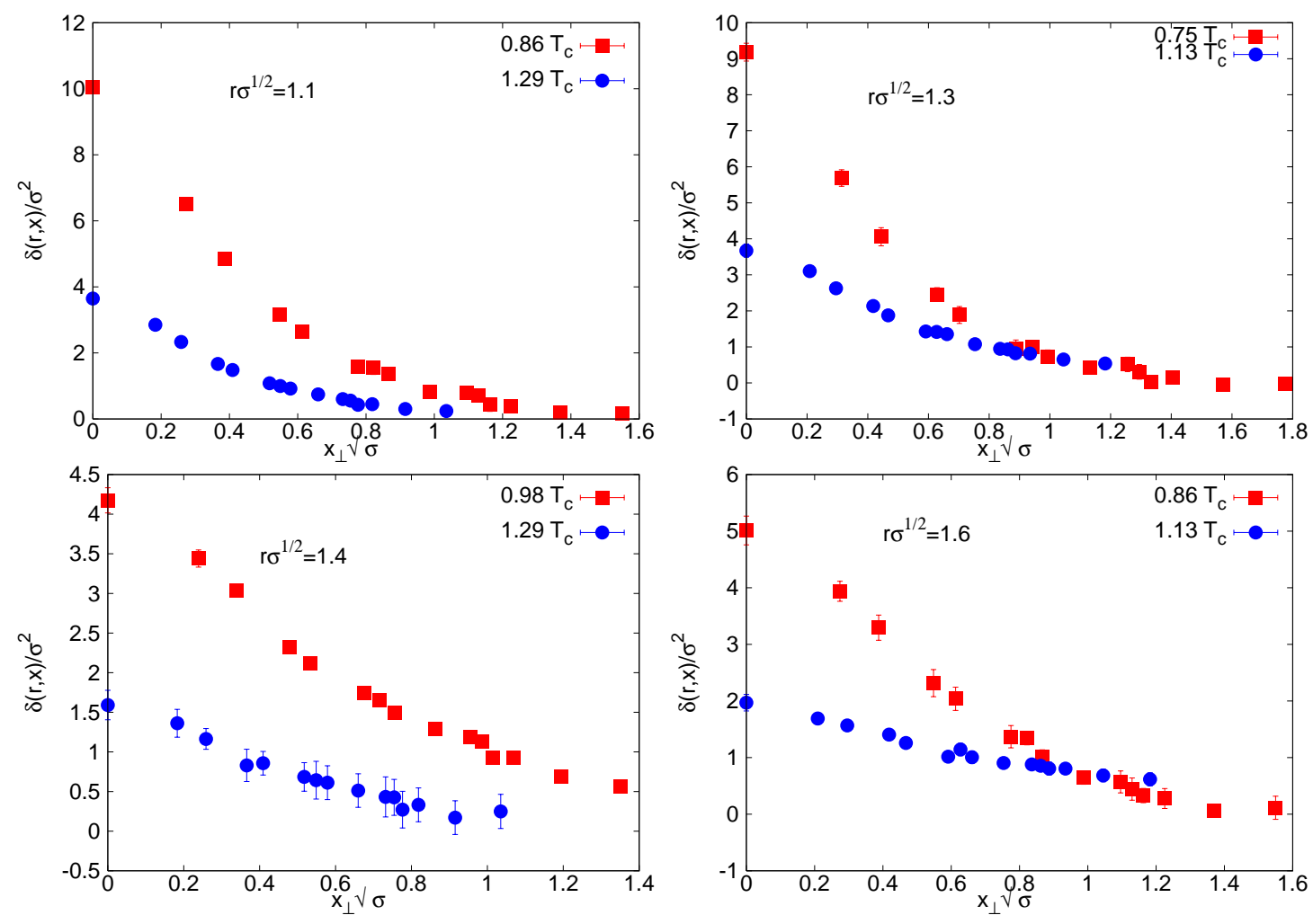

Figure 6.26.: Transverse profile $\delta\left(x_{\|}=r / 2, x_{\perp}\right)$ at $r \sqrt{\sigma}=1.1,1.3,1.4$ and 1.6 for several values of temperatures from $24 \times 12^{2} \times 6$ lattice. 


\subsection{Fit to the Transverse Distribution}

In principle, the width of the flux tube will be different at each $x_{\|}$along the axis connecting the $q \bar{q}$ pair. However, we prefer to choose a middle transverse plane $\left(x_{\|}=r / 2, x_{\perp}\right)$. The data values on the middle transverse plane allow for fits to estimate half width squared, $D^{2}$, of the flux tube.

All transverse profiles show a general behavior that they have a maximum value at $x_{\perp}=0$, then they decrease with transverse distance $x_{\perp}$ rapidly. We have seen from Fig.

6.5 that all our data obey

$$
\frac{1}{2} E_{\perp}^{2}\left(r, x_{\perp}\right) \approx-\frac{1}{2} B_{\perp}^{2}\left(r, x_{\perp}\right) \approx-\frac{1}{2} B_{\|}^{2}\left(r, x_{\perp}\right)
$$

Hence we fit the average of the three components $\frac{1}{2} E_{\perp}^{2}\left(r, x_{\perp}\right),-\frac{1}{2} B_{\perp}^{2}\left(r, x_{\perp}\right)$ and $-\frac{1}{2} B_{\|}^{2}\left(r, x_{\perp}\right)$ and $1 / 2 E_{\|}^{2}\left(x_{\|}\right)$on the center plane between the $q \bar{q}$ pair, for each $\beta$ value, to an exponential

$$
f_{\exp }\left(x_{\perp}\right)=a_{1} \exp \left(-a_{2} x_{\perp}\right)
$$

and a coulombic

$$
f_{\text {coul }}\left(x_{\perp}\right)=a_{1} /\left(a_{2}+x_{\perp}^{2}\right)^{3}
$$

fit function. It turned out that the average of the three components is adequately described by the exponential fit function while $1 / 2 E_{\|}^{2}$ in addition needs a coulombic contribution. Thus, the fitted form [1] is

$$
\begin{gathered}
\frac{1}{2 \beta} E_{\perp}^{2}\left(r, x_{\perp}\right) \approx-\frac{1}{2 \beta} B_{\|}^{2}\left(r, x_{\perp}\right) \approx-\frac{1}{2 \beta} B_{\perp}^{2}\left(r, x_{\perp}\right)=a_{1} e^{-a_{2} x_{\perp}}, \\
\frac{1}{2 \beta} E_{\|}^{2}\left(r, x_{\perp}\right)=\frac{a_{1}}{\left(a_{2}+x_{\perp}^{2}\right)^{3}}+a_{1}^{(\varepsilon)} e^{-a_{2}^{(\varepsilon)} x_{\perp}} .
\end{gathered}
$$

From the resulting fit parameters we determine the width of the flux tube defined via $[1]$

$$
D_{\varepsilon, \delta} / a=\sqrt{\frac{\int d^{2} x_{\perp} x_{\perp}^{2}\left(E^{2} \pm B^{2}\right)}{\int d^{2} x_{\perp}\left(E^{2} \pm B^{2}\right)}} .
$$




\begin{tabular}{|c|c||c|c|c|c|c|c|c|c|c|}
\hline$\beta$ & $r / a$ & $a_{1}$ & $a_{2}$ & $a_{1}^{(\varepsilon)}$ & $a_{2}^{(\varepsilon)}$ & $a^{-2} D_{\varepsilon}^{2}$ & $a^{-2} D_{\delta}^{2}$ & $a E_{s}$ & $\chi^{2}$ & $<\chi^{2}>$ \\
\hline \hline 2.20 & 2 & $0.12(4)$ & $2.45(26)$ & $0.0834(8)$ & $2.27(4)$ & $1.16(4)$ & $1.99(14)$ & $0.223(8)$ & 419 & 26 \\
& 4 & $0.25(9)$ & $2.90(46)$ & $0.018(2)$ & $2.98(1.48)$ & $0.68(67)$ & $2.80(42)$ & $0.028(28)$ & 166 & 10 \\
\hline 2.25 & 2 & $2.91(7)$ & $7.51(8)$ & $0.0618(5)$ & $2.21(2)$ & $1.23(2)$ & $6.63(6)$ & $0.178(4)$ & 244 & 15 \\
& 4 & $8.31(42)$ & $10.93(24)$ & $0.012(1)$ & $0.99(7)$ & $6.12(87)$ & $10.43(20)$ & $0.173(28)$ & 2.6 & 0.2 \\
\hline 2.27 & 2 & $1.28(3)$ & $4.80(4)$ & $0.0463(4)$ & $2.53(2)$ & $0.94(1)$ & $4.49(3)$ & $0.103(2)$ & 422 & 26 \\
& 4 & $25.07(5.98)$ & $17.92(1.73)$ & $0.0099(5)$ & $1.26(16)$ & $3.78(96)$ & $17.21(1.52)$ & $0.089(23)$ & 11 & 1 \\
\hline 2.29 & 2 & $23.83(1.15)$ & $17.18(32)$ & $0.0448(4)$ & $2.32(2)$ & $1.11(2)$ & $16.15(27)$ & $0.119(2)$ & 807 & 50 \\
& 4 & $35.91(9.14)$ & $23.57(2.59)$ & $0.0071(4)$ & $1.03(8)$ & $5.65(88)$ & $22.41(2.20)$ & $0.09(2)$ & 6.3 & 0.4 \\
\hline 2.30 & 2 & $3.83(21)$ & $9.35(18)$ & $0.0400(3)$ & $2.34(2)$ & $1.09(2)$ & $8.52(14)$ & $0.106(2)$ & 529 & 33 \\
& 4 & & & & & & & & & \\
\hline 2.32 & 2 & $4.01(12)$ & $10.23(11)$ & $0.0322(2)$ & $2.23(2)$ & $1.21(2)$ & $9.32(9)$ & $0.094(2)$ & 353 & 22 \\
& 4 & & & & & & & & & \\
\hline
\end{tabular}

Table 6.3.: The fit parameters and the derived quantities on the lattice $16 \times 8^{2} \times 4$.

The width of the energy and action density, corresponding to the $+/-$ sign, are denoted by an index $\varepsilon$ and $\delta$ respectively. In terms of the fit parameters they are given by

$$
\begin{gathered}
a^{-2} D_{\varepsilon}^{2}=\frac{6}{\left(a_{2}^{(\varepsilon)}\right)^{2}} \\
a^{-2} D_{\delta}^{2}=\frac{6 a_{1}^{(\varepsilon)}\left(a_{2}^{(\varepsilon)}\right)^{-4}+6 a_{1} / 4 a_{2}}{a_{1}^{(\varepsilon)}\left(a_{2}^{(\varepsilon)}\right)^{-2}+6 a_{1} / 4 a_{2}^{2}} .
\end{gathered}
$$

A further quantity of interest is the total energy in a slice of thickness one lattice unit:

$$
a E_{s}=\int d^{2} x_{\perp}\left(E^{2}+B^{2}\right) / 2
$$

and it is given by the fit parameters as

$$
a E_{s}=\frac{2 \pi \beta a_{1}^{(\varepsilon)}}{\left(a_{2}^{(\varepsilon)}\right)^{2}} .
$$

The $\chi^{2}$ fit results on the four fit parameters and on the derived quantities from $16 \times 8^{2} \times 4$ and $24 \times 12^{2} \times 6$ lattices are listed in Tables. 6.3 and 6.4, respectively, together with their statistical errors in brackets and corresponding $\chi^{2}$ values in the last two columns.

Since we did not prefer to do an interpolation on our data we had to choose those $r=N a$ separations with $N$ to be of an even number in order to fit the transverse profiles on $x_{\|}=r / 2$. The lattice $q \bar{q}$ separations fitted are written in the first columns named $r / a$ of the tables.

It turned out that the fit does not work well when $r$ increases as well as for smaller $\beta$. Not well fitted ones are left open in the tables. The displayed derived quantities are 


\begin{tabular}{|c|c||c|c|c|c|c|c|c|c|c|}
\hline$\beta$ & $r / a$ & $a_{1}$ & $a_{2}$ & $a_{1}^{(\varepsilon)}$ & $a_{2}^{(\varepsilon)}$ & $a^{-2} D_{\varepsilon}^{2}$ & $a^{-2} D_{\delta}^{2}$ & $a E_{s}$ & $\chi^{2}$ & $\left\langle\chi^{2}>\right.$ \\
\hline \hline 2.31 & 4 & $1.43(69)$ & $6.11(96)$ & $0.0117(5)$ & $1.15(11)$ & $4.53(87)$ & $5.90(78)$ & $0.13(3)$ & 104 & 4 \\
& 6 & $36.7(65.1)$ & $22.3(13.8)$ & $0.008(1)$ & $0.44(18)$ & $30.99(25.35)$ & $24.66(15.05)$ & $0.60(49)$ & 55 & 2 \\
\hline 2.35 & 4 & $6.38(2.03)$ & $11.91(1.27)$ & $0.0116(5)$ & $1.23(14)$ & $3.96(90)$ & $11.09(1.01)$ & $0.11(2)$ & 26 & 1 \\
& 6 & & & & & & & & \\
\hline 2.39 & 4 & $3.43(21)$ & $10.38(24)$ & $0.0050(2)$ & $1.19(4)$ & $4.24(28)$ & $9.96(21)$ & $0.053(4)$ & 53 & 2 \\
& 6 & $9.24(2.99)$ & $19.51(2.41)$ & $0.0018(2)$ & $0.83(14)$ & $8.71(2.94)$ & $18.79(2.09)$ & $0.039(14)$ & 12 & 1 \\
\hline 2.43 & 4 & $3.08(53)$ & $14.08(94)$ & $0.0034(1)$ & $1.04(4)$ & $5.55(43)$ & $13.06(73)$ & $0.048(1)$ & 56 & 2 \\
& 6 & $24.8(2.7)$ & $33.5(1.5)$ & $0.00090(7)$ & $0.93(5)$ & $6.93(75)$ & $32.7(1.39)$ & $0.016(2)$ & 88 & 3 \\
\hline 2.47 & 4 & $2.51(9)$ & $13.86(22)$ & $0.00204(9)$ & $1.33(6)$ & $3.39(31)$ & $13.28(19)$ & $0.018(2)$ & 130 & 5 \\
& 6 & $15.99(79)$ & $37.4(79)$ & $0.00058(7)$ & $0.98(8)$ & $6.25(1.02)$ & $36.34(74)$ & $0.009(2)$ & 66 & 3 \\
\hline 2.51 & 4 & $0.72(2)$ & $12.53(16)$ & $0.00200(4)$ & $1.11(4)$ & $4.87(35)$ & $11.06(11)$ & $0.026(2)$ & 36 & 1 \\
& 6 & $0.44(8)$ & $11.68(85)$ & $0.00024(2)$ & $1.33(31)$ & $3.39(1.58)$ & $11.45(79)$ & $0.002(1)$ & 192 & 7 \\
\hline
\end{tabular}

Table 6.4.: The fit parameters and the derived quantities on the lattice $24 \times 12^{2} \times 6$.

$a^{-2} D_{\varepsilon}^{2}, a^{-2} D_{\delta}^{2}$ and $a E_{s}$ but plotted ones are $D_{\varepsilon}^{2}, D_{\delta}^{2}$ and $E_{s}$ in units of the string tension after their scaling according to the scaling function. The resulting half widths for energy and action densities will be discussed in the next section.

As a by-product we obtain the total energy in a slice of thickness of the flux tube given by Eq. 6.8 from the fit of the transverse profiles. (See 9th column of the tables).

In Fig. 6.27 we show the results for $E_{s}$ from the $N_{\tau}=6$ lattice. The figure shows that $E_{s}$ is decreasing with temperature. Note also that the results have been plotted at fixed distance in lattice units. This quantity resembles the string tension as it expresses an energy per slice of the flux tube. The fact that $E_{s}$ decreases, therefore, agrees with our results on the temperature dependent string tension presented and discussed in Sec. 3.1 of Chapter 3.

\subsection{Width of the Flux Tube}

We call the width of the energy density the physical width of the flux tube. It was already clearly seen from the transverse profiles shown in Figs. 6.25 and 6.26 that the half width of the flux tube decreases with increasing temperature. In order to see its $T$-dependence more quantitatively we have fitted the transverse profiles of the field components to the fit function given by Eq. 6.4 for each $\beta$ value.

The width of the energy $D_{\varepsilon}^{2}$ and action density $D_{\delta}^{2}$ as determined from the fits of the $N_{\tau}=6$ data are plotted in Fig. 6.28 at several lattice $q \bar{q}$ separations. 


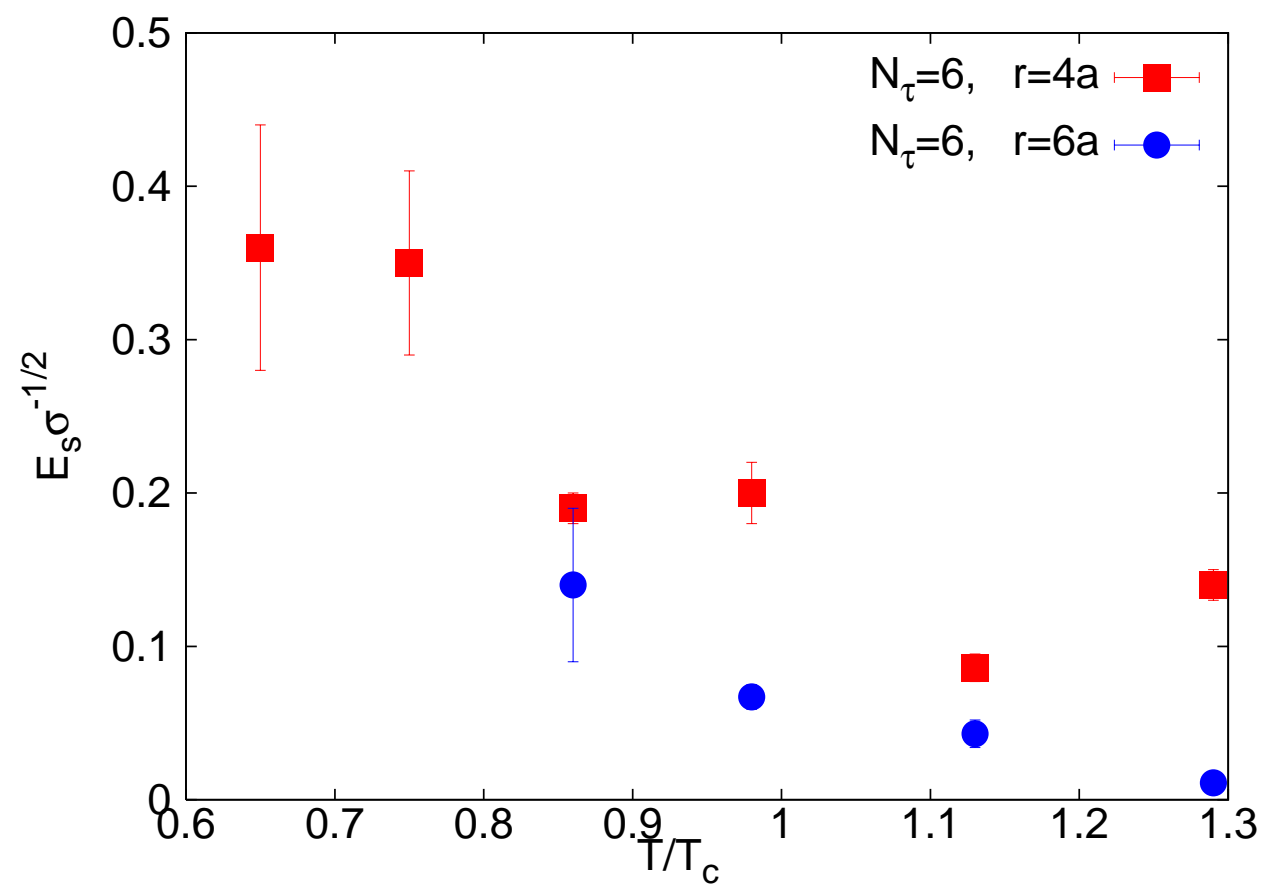

Figure 6.27.: The energy in a slice of thickness of the flux tube, $E_{s}$, in units of the string tension as determined from the fit on the lattice $24 \times 12^{2} \times 6$.
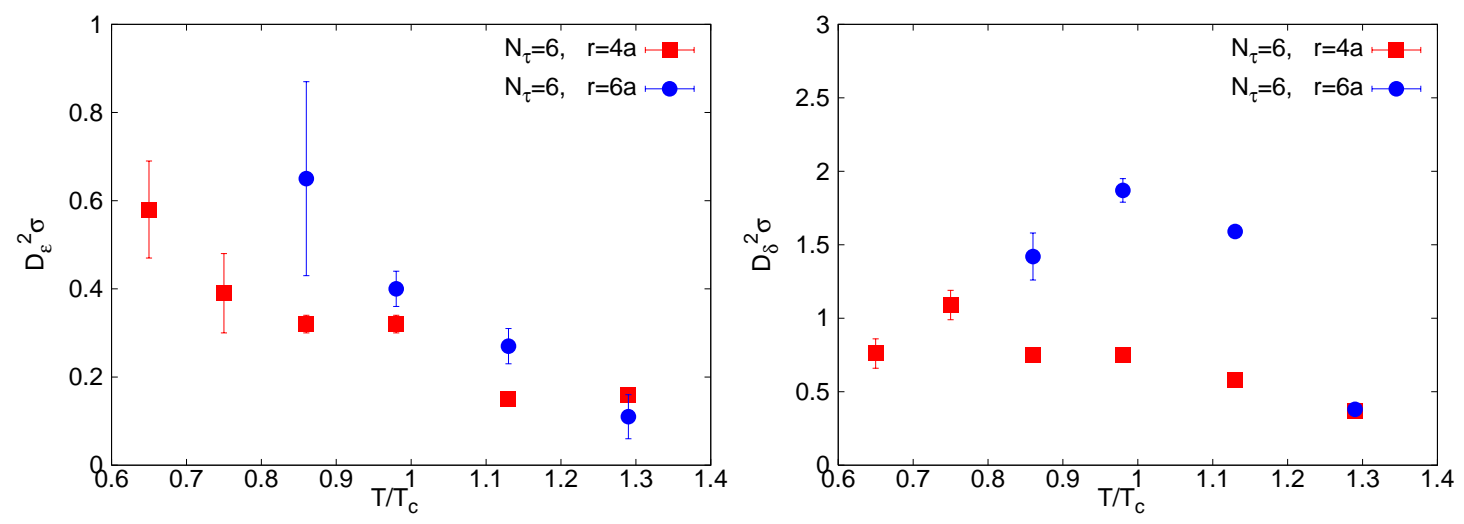

Figure 6.28.: Left: The mean squared width of the energy density, $D_{\varepsilon}^{2}$, and $\boldsymbol{R i g h t}$ : The mean squared width of the action density, $D_{\delta}^{2}$, in units of the string tension as a function of temperature at $r=4 a$ and $6 a$ from $24 \times 12^{2} \times 6$ lattice. 
In the left of Fig. 6.28 we see the mean squared width of the energy density, $D_{\varepsilon}^{2}$, versus temperature at lattice $q \bar{q}$ separations $r=4 a$ and $6 a$ from the lattice of size $24 \times 12^{2} \times 6$. At both values of the separation one can say $D_{\varepsilon}^{2}$ is decreasing with increasing temperature. For the width of the action density in the right of Fig. $6.28 r=4 a$ appears decreasing with temperature, while $r=6 a$ seems to increase up to $T_{c}$. The difference of values of the width at $r=4 a$ and $6 a$ can be considered not significantly large. Unfortunately, the fit is not working for $r=6 a$ at small $\beta$. However, the general $T$-dependence is a decreasing dependence. We also confirm the width of the action flux tube to be larger than the width of the energy flux tube.

\subsection{Disappearance of The Flux Tube above $T_{c}$}

At finite temperatures the flux tube is expected to exist only in the region below $T_{c}$, but not in the high-temperature deconfined phase. In this section we shall collect our observations of disappearance or melting of the flux tube as temperature comes close to the critical temperature of the deconfinement transition.

A first direct confirmation of our expectation was the decrease of the values of the field strengths on the middle point between the $q \bar{q}$ pair as temperature increases.

This falling down of the field strengths on the middle point was already observed when we discussed the longitudinal profiles as a function of temperature in Figs. 6.10, 6.11, 6.12 and 6.13 in Sec. 6.2. In Fig. 6.29 of this section, all field strengths as well as the energy and action densities on the middle point are collected in one plot as a function of temperature at $r=4 a, 6 a$ and $8 a . q \bar{q}$ separation and the data are in lattice units in this figure.

One should notice from the figure that at the larger separation and at the smaller $\beta$ the data have relatively large error bars sometimes having the negative values. This happens at one temperature value $T=0.65 T_{c}$ for $r=6 a$ and at two temperatures $T=0.65 T_{c}$ and $0.75 T_{c}$ for $r=8 a$ tending to increase the number of $\beta$ at which the data are noisy with increasing lattice separation. As $\beta$ increases $T$ rises and we get more stable data values with small error bars. 

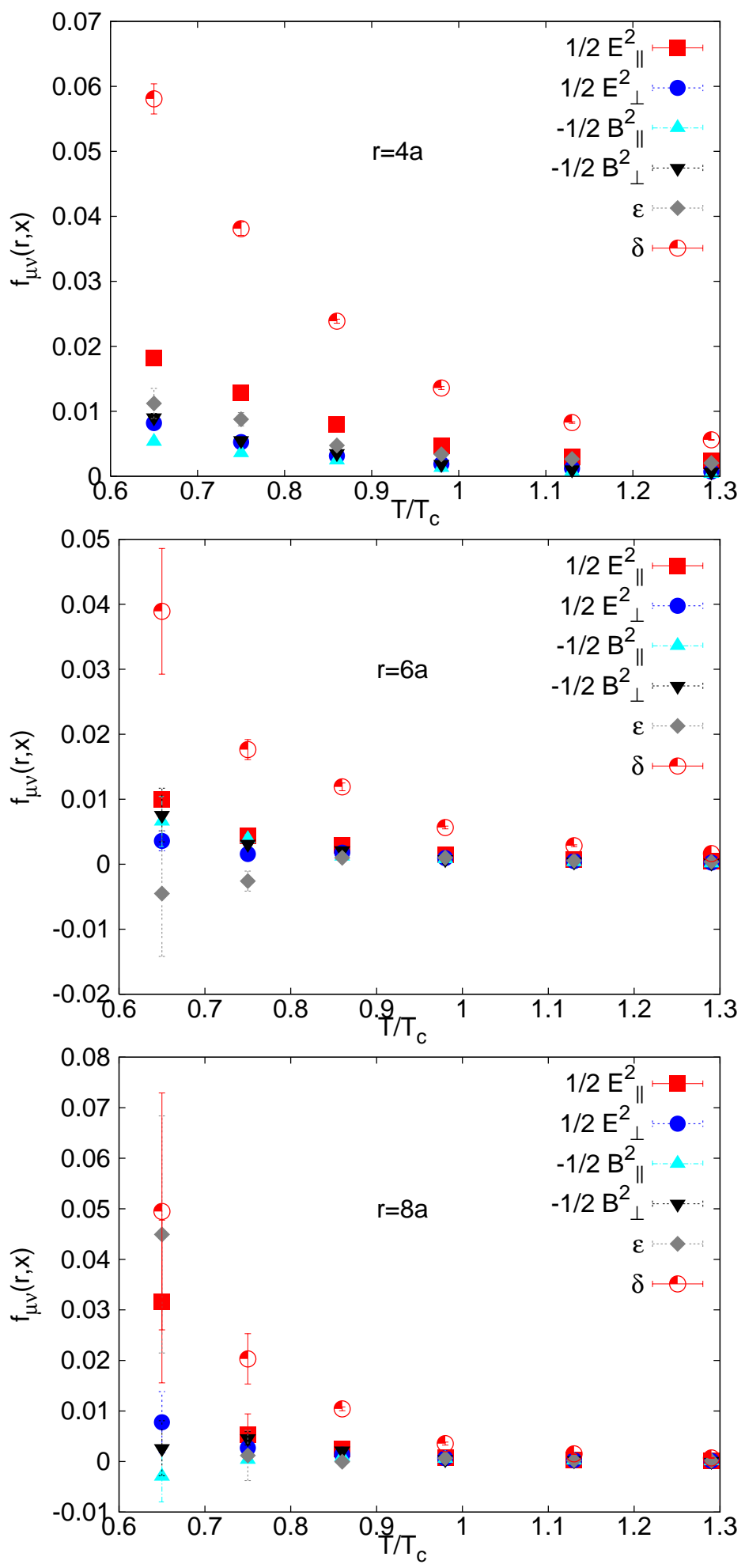

Figure 6.29.: Field strengths on the middle point between the $q \bar{q}$ pair as a function of temperature at $q \bar{q}$ separations $r=4 a, 6 a$ and $8 a$. Lattice size is $24 \times 12^{2} \times 6$. The data are in lattice units. 


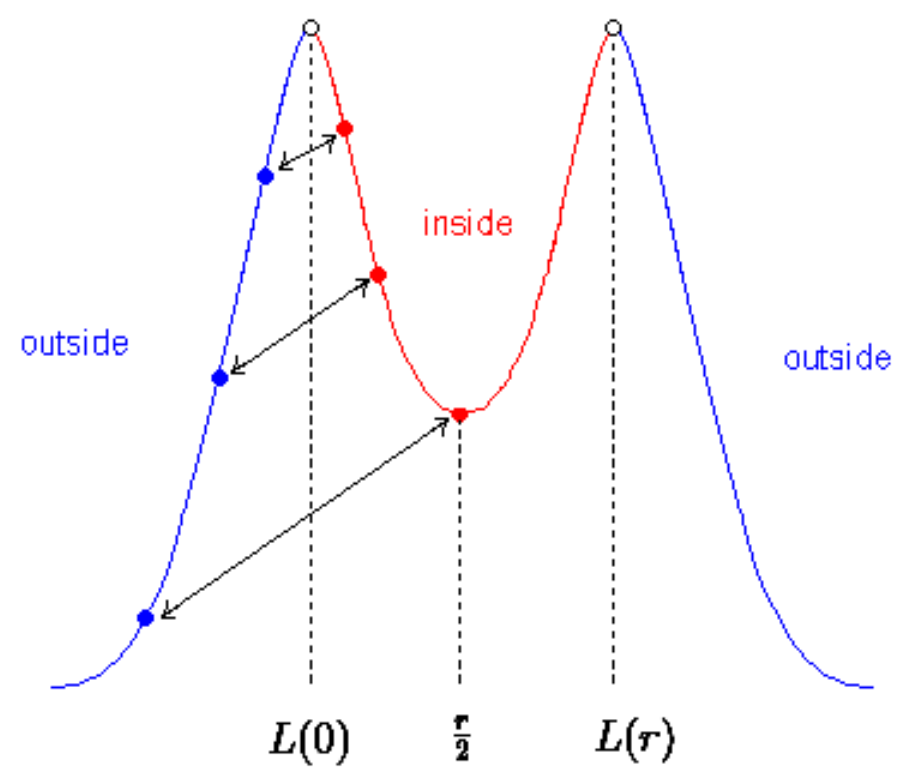

Figure 6.30.: A longitudinal profile of the flux tube. Outside of the flux tube is drawn by the blue line and inside one is drawn by the red line.

All the field strengths as well as the energy and action densities are all vanishing with increasing temperature in Fig. 6.29. The field strengths, except for $\delta$, are almost becoming zero at about $T=0.98 T_{c}$ for $r=4 a$, while the same is happening at $T=0.86 T_{c}$ for $r=6 a$ and at $T=0.75 T_{c}$ for $r=8 a$. This means that an increase of the separation accelerates the disappearance of the flux tube. At the larger separation the lower temperature is needed the flux tube to be melted.

Next, we are going to introduce the notion of inside and outside of the flux tube to also show the melting of the flux tube. In the figures of the longitudinal profiles, $x_{\|}=0$ is the location of one Polyakov loop, so $x_{\|}<0$ and $x_{\|}>r$ correspond to the outside region of the flux tube, $0<x_{\|}<r$ correspond to the inside region of the flux tube as illustrated in Fig. 6.30.

So computing the difference of field strength values

$$
\Delta E_{\|}^{2}(r, x)=\frac{1}{2} E_{\|}^{2}\left(r, x_{\|}=n a, x_{\perp}=0\right)-\frac{1}{2} E_{\|}^{2}\left(r, x_{\|}=-n a, x_{\perp}=0\right)
$$

and similarly $\Delta E_{\perp}^{2}(r, x), \Delta B_{\|}^{2}(r, x)$ and $\Delta B_{\perp}^{2}(r, x)$ as well as of energy and action densities

$$
\Delta \varepsilon(r, x)=\varepsilon\left(r, x_{\|}=n a, x_{\perp}=0\right)-\varepsilon\left(r, x_{\|}=-n a, x_{\perp}=0\right),
$$




$$
\Delta \delta(r, x)=\delta\left(r, x_{\|}=n a, x_{\perp}=0\right)-\delta\left(r, x_{\|}=-n a, x_{\perp}=0\right)
$$

on an inside point that is at the lattice distance $x_{\|}=n a$ from $L(0)$ and an outside one at $x_{\|}=-n a$ one can watch the disappearance of the flux tube with increasing temperature. The differences in Eq. 6.1 as a function of temperature are plotted in Fig. 6.31, where $n$ takes values 1 and 2 for $r=4 a$, while it takes 1,2 and 3 for $r=6 a$. The number of lattice sites the difference of which is computed increases when the $q \bar{q}$ lattice separation increases.

The difference of the field strengths values on inside and outside points decreases and the two values at a given temperature are getting close to each other with increasing temperature. This means that the field strength distribution approaches the one of a single isolated quark with rising temperature. This holds for all field components as well as the energy and action density distributions as illustrated in Fig. 6.31 and 6.32. The statement that an increase of the $q \bar{q}$ separation accelerates the difference of field strength values going to zero should be repeated here.

By doing the detailed quantitative estimation of the fall of the field strengths on the middle point and of the thinning of the width we are driven to the conclusion that the flux tube disappears as it undergoes a phase transition. 

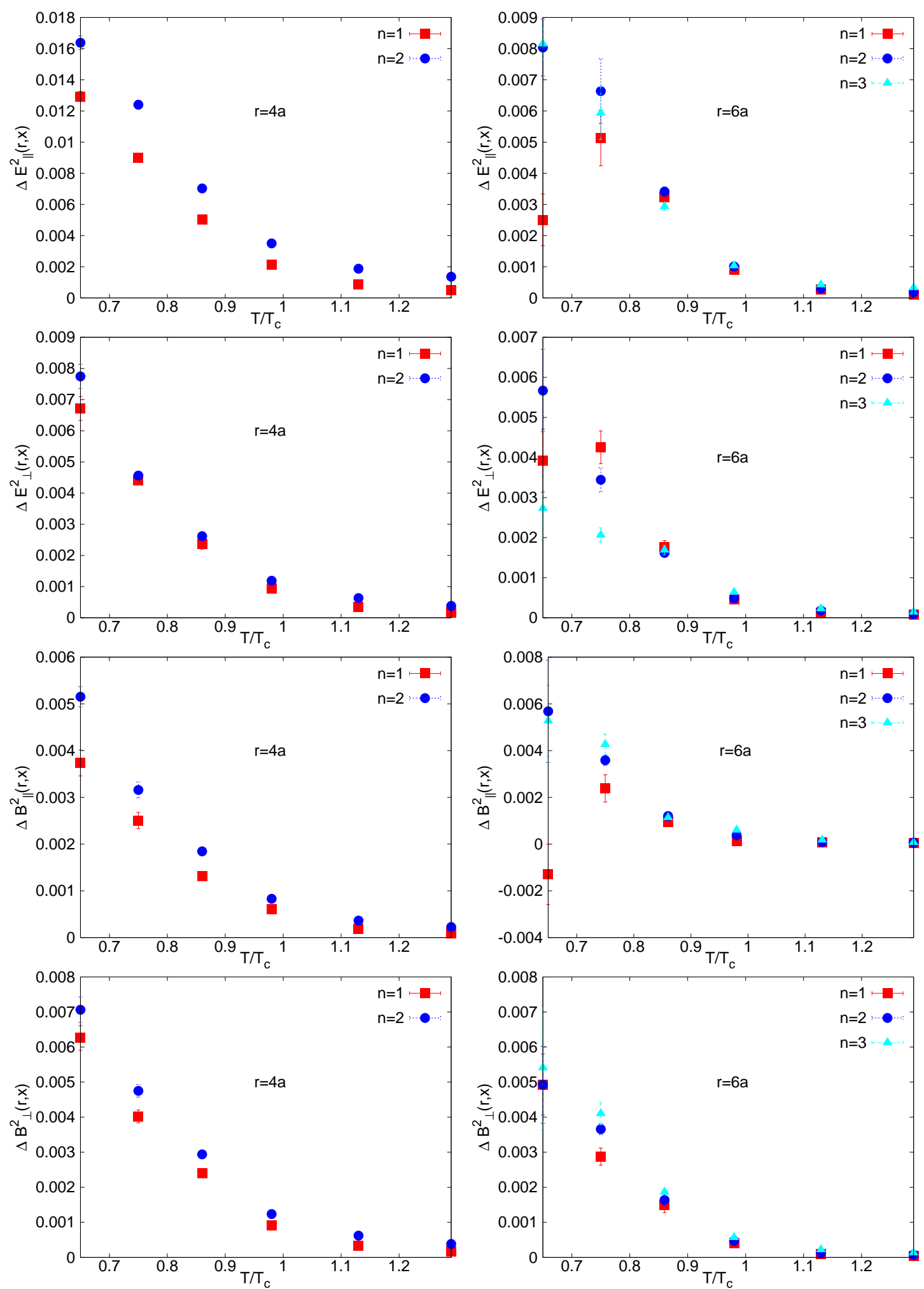

Figure 6.31.: Difference of field strength values on inside and outside points of the flux tube as a function of $T$ from $24 \times 12^{2} \times 6$ lattice. Left: $r=4 a ;$ Right: $r=6 a$. 

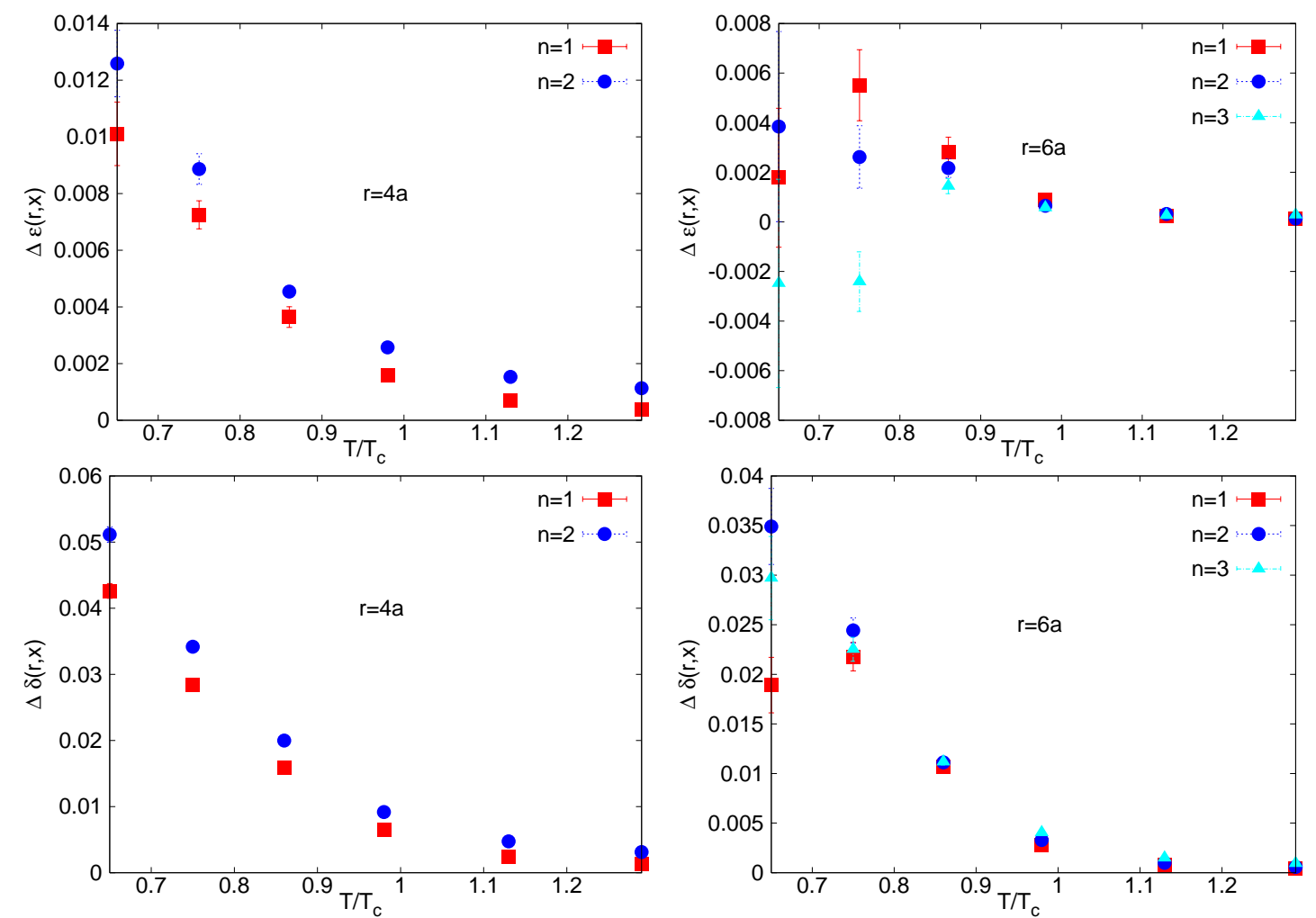

Figure 6.32.: Difference of energy and action densities on inside and outside points of the flux tube as a function of $T$ from $24 \times 12^{2} \times 6$ lattice. Left: $r=4 a$; Right: $r=6 a$. 


\section{Conclusions}

There are numerical indications that the slope of the linearly rising heavy quark potential decreases as temperature increases due to the temperature dependent string tension. Above $T_{c}$, the potential is exponentially screened. Motivated by the decrease of the potential with temperature, we were interested in how the flux tube connecting quarks reacts when the temperature changes. In particular, we were interested in the $T$-dependence of the shape and size of the tube.

Therefore, in this work we have studied field distributions around a static $q \bar{q}$ pair at high temperature using Polyakov loop plaquette correlations at physical $q \bar{q}$ separations up to $1.4 \mathrm{fm}$. We simulated pure gauge theory with gauge group $S U(2)$ on lattices of size $8^{3} \times 4,16 \times 8^{2} \times 4$ and $24 \times 12^{2} \times 6$. The temperature range was $0.65 T_{c}-1.3 T_{c}$. Measuring the electric and magnetic components of the field in close vicinity of the phase transition by using Monte Carlo numerical simulation, we were able to see longitudinal and transverse profiles of the flux tube in the vicinity of the critical temperature.

Distribution surfaces obtained for the field strengths show the expected general feature that they are symmetric with respect to the $q \bar{q}$ axis as well as the middle plane between the $q \bar{q}$ pair. We see the two peaks at the two quark sources and the field strengths decreasing as the plaquette goes away from the sources.

We conclude that there are three factors that lead the field strength value to decrease when the values of those factors or parameters increase, plaquette distance $x, q \bar{q}$ separation $r$ and temperature $T$. We have investigated the influence of the latest one in more detail, focusing on whether the width of the flux tube increases or decreases with rising temperature.

First observation from the results for the longitudinal as well as the transverse profiles was that the parallel electric field component turned out to be larger than the other 
three field components, which are approximately equal, at a fixed constant $q \bar{q}$ separation and temperature. This tells us that, indeed, the force that comes from the field strength that is oriented along the axis connecting the $q \bar{q}$ pair is large and trying to bind a quark and an antiquark together. This was true for all lattice sizes.

We have analyzed how the longitudinal and transverse profiles of all four field components which compose energy as well as action densities in the flux tube change as a function of temperature at various $q \bar{q}$ separations. It has been found that it becomes difficult to obtain the data at as large as possible $q \bar{q}$ separations one wants. The signal in the correlations between a plaquette and Polyakov loops tends to get drowned in the statistical noise when the distance between two Polyakov loops increases. We could reduce large statistical errors at large $q \bar{q}$ separation using the link integration method, which also results in saving computing time. In order to get the same accuracy on the average values without the link integration, the simulation would require more measurements than the one with the link integration.

What was mainly concluded from the longitudinal profiles is that the field strengths at $x_{\|}=r / 2, x_{\perp}=0$ fall down with rising temperature. This was also confirmed when we computed the differences of the field strength values on inside and outside points of the flux tube and plotted them as a function of temperature. Doing so we were able to show that the field strength distribution approaches the one of a single isolated quark when the rising temperature approaches the critical temperature.

The field strengths at $x_{\|}=r / 2, x_{\perp}=0$ which were falling down with rising temperature on the longitudinal profiles are the maximum field strengths for the transverse profiles at a certain temperature value. This means that the height of the flux tube decreases with rising temperature.

From the transverse profiles at various temperatures we further have found that the physical width of the flux tube decreases when the temperature increases.

The decrease with increasing temperature of the width and the height of the transverse profiles at the same time shows the gradual disappearance of the flux tube when the temperature approaches the critical temperature of the phase transition from the confined phase to the deconfined phase. The gradual disappearance agrees with the 
continuous behaviour of the second order phase transition of $S U(2)$ gauge theory. 


\section{Appendix}

\section{A. $S U(2)$}

A $2 \times 2$ unitary matrix with unit determinant can be written in the form

$$
U=\exp \left(i \frac{\vec{\tau}}{2} \cdot \vec{\theta}\right)
$$

where $\vec{\theta}=\left(\theta_{1}, \theta_{2}, \theta_{3}\right)$ are three arbitrary parameters and $\vec{\tau}=\left(\tau_{1}, \tau_{2}, \tau_{3}\right)$ are the traceless matrices

$$
\tau_{1}=\left(\begin{array}{cc}
0 & 1 \\
1 & 0
\end{array}\right), \quad \tau_{2}=\left(\begin{array}{cc}
0 & -i \\
i & 0
\end{array}\right), \quad \tau_{3}=\left(\begin{array}{cc}
1 & 0 \\
0 & -1
\end{array}\right)
$$

known as the Pauli matrices. They obey the commutation relations

$$
\frac{\tau_{1}}{2} \frac{\tau_{2}}{2}-\frac{\tau_{2}}{2} \frac{\tau_{1}}{2} \equiv\left[\frac{\tau_{1}}{2}, \frac{\tau_{2}}{2}\right]=i \frac{\tau_{3}}{2}
$$

and cyclic permutations. Putting

$$
U=\left(\begin{array}{ll}
a & b \\
c & d
\end{array}\right)
$$

the unitarity condition $U^{+}=U^{-1}$, reads

$$
\left(\begin{array}{ll}
a^{*} & c^{*} \\
b^{*} & d^{*}
\end{array}\right)=\left(\begin{array}{cc}
d & -b \\
-c & a
\end{array}\right)
$$

and hence $a^{*}=d, b^{*}=-c$. Then $\operatorname{det} U=|a|^{2}+|b|^{2}=1$, so we have

$$
U=\left(\begin{array}{cc}
a & b \\
-b^{*} & a^{*}
\end{array}\right), \quad|a|^{2}+|b|^{2}=1 .
$$




\section{B. Maxwell and Proca equations}

Here we derive the correspondence between the six $(\mu, \nu)$ orientations of the plaquette and the electric and magnetic components in Minkowski space.

Photons have no mass and are described by Maxwell's equations, and massive spin 1 particles are described by the Proca equations. Well known Maxwell's equations are $[34]$

$$
\begin{aligned}
\operatorname{div} \mathbf{B} & =0, \\
\operatorname{curl} \mathbf{E}+\frac{\partial \mathbf{B}}{\partial t} & =0, \\
\operatorname{div} \mathbf{E} & =\rho, \\
\operatorname{curl} \mathbf{B}-\frac{\partial \mathbf{E}}{\partial t} & =\mathbf{j} .
\end{aligned}
$$

Introducing the 4-vector potential

$$
A^{\mu}=(\phi, \mathbf{A})
$$

with

$$
\begin{array}{r}
\mathbf{B}=\operatorname{curl} \mathbf{A}, \\
\mathbf{E}=-\frac{\partial \mathbf{A}}{\partial t}-\nabla \phi,
\end{array}
$$

Eqs. (B.7) and (B.8) are automatically satisfied, since div curl $\equiv 0$ and curl grad $\equiv$ 0. Now observe that the right-hand side of Eqs. (B.12) are the components of a 4dimensional curl, defined by

$$
F^{\mu \nu}=-F^{\nu \mu}=\partial^{\mu} A^{\nu}-\partial^{\nu} A^{\mu} .
$$

It has components (recall that $\partial^{i}=-\partial_{i}$ )

$$
\begin{array}{r}
F^{0 i}=\partial^{0} A^{i}-\partial^{i} A^{0} \\
=\left(\frac{\partial \mathbf{A}}{\partial t}+\nabla \phi\right)_{i} \\
=-E^{i}
\end{array}
$$

and

$$
\begin{array}{r}
F^{i j}=\partial^{i} A^{j}-\partial^{j} A^{i} \\
=-\varepsilon^{i j k} B^{k},
\end{array}
$$


where $\varepsilon^{i j k}=\varepsilon_{i j k}$ is the totally antisymmetric Levi-Civita symbol

$\varepsilon_{i j k}=\left\{\begin{aligned}+1 & \text { if }(i j k) \text { is an even permutation of (123) } \\ -1 & \text { if }(i j k) \text { is an odd permutation of (123) } \\ 0 & \text { otherwise. }\end{aligned}\right.$

Eqs. (B.13) and (B.14) may be displayed in matrix form, with the rows and columns corresponding to the numbers $0,1,2,3$ :

$$
F^{\mu \nu}=\left(\begin{array}{cccc}
0 & -E^{1} & -E^{2} & -E^{3} \\
E^{1} & 0 & -B^{3} & B^{2} \\
E^{2} & B^{3} & 0 & -B^{1} \\
E^{3} & -B^{2} & B^{1} & 0
\end{array}\right)
$$

$F^{\mu \nu}$ is called the electromagnetic field tensor. Lowering the indices on the field tensor gives $\left(E_{x} \equiv E_{1}\right.$, etc. $)$

$$
\begin{gathered}
F_{01}=E_{x}, \quad F_{02}=E_{y}, \quad F_{03}=E_{z}, \\
F_{12}=-B_{z}, \quad F_{31}=-B_{y}, \quad F_{23}=-B_{x} .
\end{gathered}
$$

All above derivation was of course in 4-dimensional Minkowski space-time. Now the plaquette variable

$$
\square_{\mu \nu}=-\frac{1}{2}\left(F_{\mu \nu}^{E}\right)^{2}
$$

where $F_{\mu \nu}^{E}$ is the field strength tensor in Euclidean space. Taking into account the connection between time components of gauge fields

$$
A_{0}^{M}=i A_{4}^{E}
$$

as well as the connection between space components

$$
A_{k}^{M}=A_{k}^{E}
$$

it will be found that

$$
\square_{4 i}=-\frac{1}{2}\left(F_{4 i}^{E}\right)^{2}=-\frac{1}{2}\left(-i F_{0 i}^{M}\right)^{2}=\frac{1}{2}\left(F_{0 i}^{M}\right)^{2}=\frac{1}{2} E_{i}^{2}
$$

for the electric field components and that

$$
\square_{j k}=-\frac{1}{2}\left(F_{j k}^{E}\right)^{2}=-\frac{1}{2}\left(F_{j k}^{M}\right)^{2}=-\frac{1}{2} B_{i}^{2} .
$$

for the magnetic field components. Here the upper indices $E$ and $M$ denote the Euclidean and Minkowski spaces, respectively. 


\section{Link integration for $S U(2)$}

The essence of the link integration is to reduce the statistical noise by substituting the link operator by an expression giving the same mean values with less variance. We replace temporal link variables $U$ in the Polyakov loops with the new link variable $\bar{U}$ :

$$
U_{\mu}(n) \longrightarrow \bar{U}_{\mu}(n)
$$

so that

$$
\bar{U}_{\mu}(n)=\frac{\int d U_{\mu}(n) U_{\mu}(n) \exp \left(\beta \operatorname{Tr}\left(U_{\mu}(n) X_{\mu}^{\dagger}(n)\right)\right)}{\int d U_{\mu}(n) \exp \left(\beta \operatorname{Tr}\left(U_{\mu}(n) X_{\mu}^{\dagger}(n)\right)\right)}
$$

with

$$
X_{\mu}(n)=\sum_{\nu \neq \mu} U_{\nu}(n) U_{\mu}(n+\hat{\nu}) U_{\nu}^{\dagger}(n+\hat{\mu})
$$

Here

$$
U_{\mu}(n) X_{\mu}^{\dagger}(n)=\sum_{L \supset \operatorname{link} x, \mu} U_{L}
$$

In this way only those links that do not share a common plaquette can be integrated independently.

In our case of $S U(2)$ gauge theory $\bar{U}$ can be calculated analytically in terms of the modified Bessel functions. The idea is the following. If $A$ and $B$ are any observables

$$
\begin{gathered}
\left\langle A \bar{U}_{i} B\right\rangle= \\
=\frac{1}{Z} \frac{\int \prod_{j \neq i} d U_{j} d U_{i} d V_{i} A V_{i} B e^{\beta T r\left(V_{i} X_{i}^{\dagger}\right)} e^{\beta T r U_{i}}}{\int d V_{i} e^{\beta T r\left(V_{i} X_{i}^{\dagger}\right)}} \\
=\frac{1}{Z} \frac{\int \prod_{j \neq i} d U_{j} d U_{i} d V_{i} A U_{i} B e^{\beta T r\left(U_{i}+V_{i}\right) X_{i}^{\dagger}}}{\int d V_{i} e^{\beta T r\left(V_{i} X_{i}^{\dagger}\right)}} \\
=\frac{1}{Z} \int \prod_{j} d U_{j} A U_{i} B e^{\beta T r\left(U_{i} X_{i}^{\dagger}\right)}
\end{gathered}
$$




$$
=\left\langle A U_{i} B\right\rangle
$$

provided neither $A$ nor $B$ contain $U_{i}$ or a modified link whose neighborhood contains $U_{i}$ because Eq. C.27 utilizes the identity

$$
\int d U d V U \exp \left(\operatorname{Tr}\left[(U+V) X^{\dagger}\right]\right)=\int d U d V V \exp \left(\operatorname{Tr}\left[(U+V) X^{\dagger}\right]\right)
$$

Now

$$
\begin{gathered}
\bar{U}=\frac{\int d U U e^{\beta T r\left(U X^{\dagger}\right)}}{\int d V e^{\beta T r\left(V X^{\dagger}\right)}} \\
=\frac{\int d\left(U X^{\dagger}\right) U e^{\beta T r\left(U X^{\dagger}\right)}}{\int d\left(V X^{\dagger}\right) e^{\beta T r\left(V X^{\dagger}\right)}}
\end{gathered}
$$

with

$$
X X^{\dagger}=\|X\| \cdot 1=\operatorname{det} X \cdot 1=\lambda^{2} \cdot 1 \quad X \in U(2) .
$$

Then $\bar{U}$ becomes

$$
\bar{U}=\frac{1}{\lambda^{2}} X \frac{\int d\left(U X^{\dagger}\right)\left(U X^{\dagger}\right) e^{\beta T r\left(U X^{\dagger}\right)}}{\int d\left(V X^{\dagger}\right) e^{\beta T r\left(V X^{\dagger}\right)}} .
$$

Let us do the integrals separately. In the evaluation of the integrals a possible parametrization

$$
U X^{\dagger}=\lambda\left(\begin{array}{cc}
\cos (k / 2)+i \sin (k / 2) \cos \theta & \sin (k / 2) \sin \theta e^{i \phi} \\
-\sin (k / 2) \sin \theta e^{-i \phi} & \cos (k / 2)-i \sin (k / 2) \cos \theta
\end{array}\right)
$$

is used. The denominator integral becomes

$$
\int d\left(V X^{\dagger}\right) e^{\beta T r\left(V X^{\dagger}\right)}=\int_{0}^{2 \pi} \sin ^{2} \frac{k}{2} d k \int_{0}^{\pi} \sin \theta d \theta \int_{0}^{2 \pi} d \phi e^{\beta \lambda \cos \left(\frac{k}{2}\right)}=\frac{8 \pi^{2}}{\beta \lambda} I_{1}(\beta \lambda) .
$$

while for the numerator integral we are left with

$$
\int d\left(U X^{\dagger}\right)\left(U X^{\dagger}\right) e^{\beta T r\left(U X^{\dagger}\right)}=8 \pi \lambda \cdot 1 \cdot\left(-\frac{\pi}{(\beta \lambda)^{2}} I_{1}(\beta \lambda)+\frac{\pi}{\beta \lambda} \frac{\partial}{\partial(\beta \lambda)} I_{1}(\beta \lambda)\right) .
$$

Thus we have to replace the temporal links in the Polyakov loops by

$$
\bar{U}_{\mu}(n)=\frac{I_{2}(\beta \lambda)}{\lambda I_{1}(\beta \lambda)} X_{\mu}(n), \quad \lambda=\sqrt{\operatorname{det}\left(X_{\mu}(n)\right)}
$$

where $I_{n}$ denote the modified Bessel functions. 


\section{Bibliography}

[1] R. Sommer, Scaling of SU(2) Flux Distribution and Potential, Nucl. Phys. B291 (1988) 673.

[2] R. W. Haymaker and J. Wosiek, Distribution of the Color Fields Around Static Quarks: Lattice Sum Rules, Phys. Rev. D43 (1991) 2676.

[3] G. S. Bali, K. Schilling and Ch. Schlichter, Observing Long Color Flux Tubes in SU(2) Lattice Gauge Theory, Phys. Rev. D51 (1995) 5165 [hep-lat/9409005].

[4] R. W. Haymaker, V. Singh and Y. Peng, Distribution of the Color Fields Around Static Quarks: Flux Tube Profiles, Phys. Rev. D53 (1996) 389 [hep-lat/9406021].

[5] K. G. Wilson, Phys. Rev. D14 (1974) 2455.

[6] H. J. Rothe, Lattice Gauge Theories: An Introduction. World Scientific (1992).

[7] Alan M. Ferrenberg and Robert H. Swendsen, New Monte Carlo Technique for Studying Phase Transitions, Phys. Rev. Lett. 61 (1988) 2635.

[8] M. Lüscher, K. Symanzik and P. Weisz, Anomalies of the Free Loop Wave Equation in the WKB Approximation, Nucl. Phys. B173 (1980) 365.

[9] M. Lüscher, G. Münster and P. Weisz, How Thick are Chromo-Electric Flux Tubes?, Nucl. Phys. B180 [FS2] (1980) 1.

[10] J. Fingberg, U. Heller and F. Karsch, Scaling and Asymptotic Scaling in the SU(2) Gauge Theory, Nucl. Phys. B392 (1993) 493.

[11] O. Kaczmarek, F. Karsch and F. Zantow, Static Quark Anti-Quark Free Energy and the Running Coupling at Finite Temperature, BI-TP 2004/16 [hep-lat/0406036].

[12] M. Gao, Heavy-quark Potential at Finite Temperature From a String Picture, Phys. 
Rev. D40 (1989) 2708; F. Gliozzi and P. Provero, The Confining String and Its Breaking in QCD, Nucl. Phys. B556 (1999) 76.

[13] R. D. Pisarski and O. Alvarez, Strings at Finite Temperature and Deconfinement, Phys. Rev. D26 (1982) 3735.

[14] O. Kaczmarek, F. Karsch, E. Laermann and M. Lutgemeier, Heavy Quark Potentials in Quenched QCD at High Temperature, Phys. Rev. D62 (2000) 034021.

[15] J. Engels, V. Mitrjushkin, Mass Gap and Finite-Size Effects in Finite Temperature SU(2) Lattice Gauge Theory, Phys. Lett. B282 (1992) 415.

[16] J. Engels, J. Fingberg and D. Miller, Phenomenological Renormalization and Scaling Behaviour of SU(2) Lattice Gauge Theory, Nucl. Phys. B387 (1992) 501.

[17] M. Fukugita and T. Niuya, The Distribution of Chromoelectric Flux in SU(2) Lattice Gauge Theory, Phys. Lett. B132 (1983) 374.

[18] Jon W. Flower and Steve W. Otto, The Field Distribution in SU(3) Lattice Gauge Theory, Phys. Lett. B160 (1985) 128.

[19] R. W. Haymaker and Y. Peng, SU(2) Flux Distributions on Finite Lattices, Phys. Rev. D47 (1993) 5104.

[20] G. 't Hooft, High Energy Physics, ed. A. Zichichi Editorice Compositori, Bologna, 1975 .

[21] G. S. Bali, Ch. Schlichter and K. Schilling, A Ginzburg-Landau Analysis of the Colour Electric Flux Tube, Nucl. Phys. Proc. Suppl. 73 (1999) 638 [hep-lat/9809039].

[22] R. W. Haymaker, V. Singh and D. Browne, Vacuum Structure of Pure Gauge Theories on the Lattice, [hep-lat/9302011].

[23] R. W. Haymaker, J. Wosiek, Distribution of the Colour Fields Around Static Quarks: Lattice Sum Rules, Phys. Rev. D43 (1991) 2676.

[24] H. B. Nielsen and P. Olesen, Vortex-Line Models for Dual Strings, Nucl. Phys. B61 (1973) 45.

[25] M. Caselle, F. Gliozzi, U. Magnea and S. Vinti, Width of Long Colour Flux Tubes in Lattice Gauge Systems, Nucl. Phys. B460 (1996) 397. 
[26] J. Kuti, J. Polonyi and K. Szalachanyi, Monte Carlo Study of SU(2) Gauge Theory at Finite Temperature, Phys. Lett. B98 (1980) 199.

[27] L. D. McLerran and B. Svetitsky, Quark Liberation at High Temperature: A Monte Carlo Study of SU(2) Gauge Theory, Phys. Lett. B98 (1981) 195.

[28] M. Creutz, Monte Carlo Study of Quantized SU(2) Gauge Theory, Phys. Rev. D21 (1980) 2308.

[29] M. Creutz, Confinement and the Critical Dimensionality of Space-Time, Phys. Rev. Lett. Vol. 43. (1979) 553.

[30] A. D. Kennedy and B. J. Pendleton, Improved Heatbath Method for Monte Carlo Calculations in Lattice Gauge Theories, Phys. Lett. B156 (1985) 393.

[31] M. Creutz, Overrelaxation and Monte Carlo Simulation, Phys. Rev. D36 (1987) 515.

[32] Philippe de Forcrand and Oliver Jahn, Monte Carlo Overrelaxation for $S U(N)$ Gauge Theories, [hep-lat/0503041].

[33] G. Parisi, R. Petronzio and F. Rapuano, A Measurement of the String Tension Near the Continuum Limit, Phys. Lett. B128 (1983) 418.

[34] Lewis. H. Ryder, Quantum Field Theory Second Edition, Cambridge University Press (1996). 


\section{Acknowledgements}

I am deeply indebted to my supervisor Prof. Dr. Edwin Laermann for proposing me such an interesting project and supervising my Ph.D thesis. I thank him for being open and patient to discuss my questions all the time. Without his continuous experienced guidance and many fruitful discussions I could not have brought this thesis to a successful conclusion.

I am extraordinary grateful to Prof. Dr. Frithjof Karsch for providing me the opportunity to collaborate with his amazing group of lattice physicists. He provided the very first nice opportunity to study in Bielefeld University.

I thank Dr. Olaf Kaczmarek, who was always open for my questions during my entire time as a Ph.D student, for teaching me so much about computers and programming languages.

I would also like to thank my officemates Dr. Sönke Wissel and Chuan Miao for the every day good atmosphere in our office. Furthermore I thank all my colleagues from the theoretical particle physics group in Bielefeld for sharing this great time with me! I also like to thank our secretaries Gudrun Eickmeyer and Susi von Reder. A very special thanks go to Gudrun Eickmeyer, who was always there for me as if she is my mother during these three years of my study.

I thank the DFG for supporting this work by funding the graduate school GRK 881 "Quantum Fields and Strongly Interacting Matter".

Finally, special thanks go to my father Dr. Chagdaa Khookhdoi, my mother Khongoroo Boroldoi and my brothers and sisters for all their love and care. I thank my husband Jambajamts Lkhamjav, my son Od-Erdem Jambajamts not only for being all the time with me but also for their understanding, invaluable help and mental support.

Sodbileg Chagdaa

Bielefeld, March 2008 